\title{
COMMON TOXICITY CRITERIA (CTC)
}

\begin{tabular}{|c|c|c|c|c|c|}
\hline \multirow[b]{2}{*}{ Adverse Event } & \multicolumn{4}{|c|}{ Grade } & \multirow[b]{2}{*}{4} \\
\hline & $\mathbf{0}$ & 1 & 2 & 3 & \\
\hline \multicolumn{6}{|c|}{ ALLERGY/IMMUNOLOGY } \\
\hline $\begin{array}{l}\text { Allergic reaction/ } \\
\text { hypersensitivity } \\
\text { (including drug fever) }\end{array}$ & none & $\begin{array}{l}\text { transient rash, drug } \\
\text { fever }<38^{\circ} \mathrm{C}\left(<100.4^{\circ} \mathrm{F}\right)\end{array}$ & $\begin{array}{l}\text { urticaria, drug fever } \\
\geq 38^{\circ} \mathrm{C}\left(\geq 100.4^{\circ} \mathrm{F}\right) \text {, } \\
\text { and/or asymptomatic } \\
\text { bronchospasm }\end{array}$ & $\begin{array}{l}\text { symptomatic } \\
\text { bronchospasm, } \\
\text { requiring parenteral } \\
\text { medication(s), with or } \\
\text { without urticaria; } \\
\text { allergy-related } \\
\text { edema/angioedema }\end{array}$ & anaphylaxis \\
\hline
\end{tabular}

Note: Isolated urticaria, in the absence of other manifestations of an allergic or hypersensitivity reaction, is graded in the DERMATOLOGY/SKIN category.

\begin{tabular}{|c|c|c|c|c|c|}
\hline $\begin{array}{l}\text { Allergic rhinitis } \\
\text { (including sneezing, nasal } \\
\text { stuffiness, postnasal drip) }\end{array}$ & none & $\begin{array}{l}\text { mild, not requiring } \\
\text { treatment }\end{array}$ & $\begin{array}{l}\text { moderate, requiring } \\
\text { treatment }\end{array}$ & - & - \\
\hline Autoimmune reaction & none & $\begin{array}{l}\text { serologic or other } \\
\text { evidence of } \\
\text { autoimmune reaction } \\
\text { but patient is } \\
\text { asymptomatic (e.g., } \\
\text { vitiligo), all organ } \\
\text { function is normal and } \\
\text { no treatment is required }\end{array}$ & $\begin{array}{l}\text { evidence of } \\
\text { autoimmune reaction } \\
\text { involving a non- } \\
\text { essential organ or } \\
\text { function (e.g., } \\
\text { hypothyroidism), } \\
\text { requiring treatment } \\
\text { other than } \\
\text { immunosuppressive } \\
\text { drugs }\end{array}$ & $\begin{array}{l}\text { reversible autoimmune } \\
\text { reaction involving } \\
\text { function of a major } \\
\text { organ or other adverse } \\
\text { event (e.g., transient } \\
\text { colitis or anemia), } \\
\text { requiring short-term } \\
\text { immunosuppressive } \\
\text { treatment }\end{array}$ & $\begin{array}{l}\text { autoimmune reaction } \\
\text { causing major grade } 4 \\
\text { organ dysfunction; } \\
\text { progressive and } \\
\text { irreversible reaction; } \\
\text { long-term } \\
\text { administration of high- } \\
\text { dose immuno- } \\
\text { suppressive therapy } \\
\text { required }\end{array}$ \\
\hline
\end{tabular}

Also consider Hypothyroidism, Colitis, Hemoglobin, Hemolysis.

Serum sickness none - - - n presen

Urticaria is graded in the DERMATOLOGY/SKIN category if it occurs as an isolated symptom. If it occurs with other manifestations of allergic or hypersensitivity reaction, grade as Allergic reaction/hypersensitivity above.

\begin{tabular}{|llllll}
\hline Vasculitis & none & $\begin{array}{l}\text { mild, not requiring } \\
\text { treatment }\end{array}$ & $\begin{array}{l}\text { symptomatic, requiring } \\
\text { medication }\end{array}$ & requiring steroids & $\begin{array}{l}\text { ischemic changes or } \\
\text { requiring amputation }\end{array}$ \\
\hline $\begin{array}{l}\text { Allergy/Immunology - Other } \\
\text { (Specify, none }\end{array}$ & mild & moderate & severe & $\begin{array}{l}\text { life-threatening or } \\
\text { disabling }\end{array}$ \\
\hline
\end{tabular}

AUDITORY/HEARING

Conductive hearing loss is graded as Middle ear/hearing in the AUDITORY/HEARING category.

Earache is graded in the PAIN category.

External auditory canal norma

$\begin{array}{llll}\text { external otitis with } & \begin{array}{l}\text { external otitis with } \\ \text { erythema or dry }\end{array} & \begin{array}{l}\text { external otitis with } \\ \text { discharge, mastoiditis }\end{array} & \begin{array}{l}\text { necrosis of the canal } \\ \text { soft tissue or bone }\end{array}\end{array}$

desquamation

Note: Changes associated with radiation to external ear (pinnae) are graded under Radiation dermatitis in the DERMATOLOGY/SKIN category. 
CTC Version 2.0

Publish Date: April 30, 1999

\begin{tabular}{|c|c|c|c|c|c|}
\hline \multicolumn{6}{|c|}{ Grade } \\
\hline Adverse Event & $\mathbf{0}$ & $\mathbf{1}$ & 2 & 3 & 4 \\
\hline Inner ear/hearing & normal & $\begin{array}{l}\text { hearing loss on } \\
\text { audiometry only }\end{array}$ & $\begin{array}{l}\text { tinnitus or hearing loss, } \\
\text { not requiring hearing } \\
\text { aid or treatment }\end{array}$ & $\begin{array}{l}\text { tinnitus or hearing loss, } \\
\text { correctable with hearing } \\
\text { aid or treatment }\end{array}$ & $\begin{array}{l}\text { severe unilateral or } \\
\text { bilateral hearing loss } \\
\text { (deafness), not } \\
\text { correctable }\end{array}$ \\
\hline Middle ear/hearing & normal & $\begin{array}{l}\text { serous otitis without } \\
\text { subjective decrease in } \\
\text { hearing }\end{array}$ & $\begin{array}{l}\text { serous otitis or infection } \\
\text { requiring medical } \\
\text { intervention; subjective } \\
\text { decrease in hearing; } \\
\text { rupture of tympanic } \\
\text { membrane with } \\
\text { discharge }\end{array}$ & $\begin{array}{l}\text { otitis with discharge, } \\
\text { mastoiditis or } \\
\text { conductive hearing loss }\end{array}$ & $\begin{array}{l}\text { necrosis of the canal } \\
\text { soft tissue or bone }\end{array}$ \\
\hline $\begin{array}{l}\text { Auditory/Hearing - Other } \\
\text { (Specify, }\end{array}$ & normal & mild & moderate & severe & $\begin{array}{l}\text { life-threatening or } \\
\text { disabling }\end{array}$ \\
\hline \multicolumn{6}{|c|}{ BLOOD/BONE MARROW } \\
\hline Bone marrow cellularity & normal for age & $\begin{array}{l}\text { mildly hypocellular or } \\
\leq 25 \% \text { reduction from } \\
\text { normal cellularity for } \\
\text { age }\end{array}$ & $\begin{array}{l}\text { moderately hypocellular } \\
\text { or }>25-\leq 50 \% \\
\text { reduction from normal } \\
\text { cellularity for age or }>2 \\
\text { but }<4 \text { weeks to } \\
\text { recovery of normal } \\
\text { bone marrow cellularity }\end{array}$ & $\begin{array}{l}\text { severely hypocellular or } \\
>50-\leq 75 \% \text { reduction } \\
\text { in cellularity for age or } \\
4-6 \text { weeks to recovery } \\
\text { of normal bone marrow } \\
\text { cellularity }\end{array}$ & $\begin{array}{l}\text { aplasia or }>6 \text { weeks to } \\
\text { recovery of normal } \\
\text { bone marrow cellularity }\end{array}$ \\
\hline \multicolumn{6}{|l|}{ Normal ranges: } \\
\hline children $(\leq 18$ years $)$ & $\begin{array}{l}90 \% \text { cellularity } \\
\text { average }\end{array}$ & & & & \\
\hline younger adults (19-59) & $\begin{array}{l}60-70 \% \\
\text { cellularity average }\end{array}$ & & & & \\
\hline older adults ( $\geq 60$ years) & $\begin{array}{l}50 \% \text { cellularity } \\
\text { average }\end{array}$ & & & & \\
\hline CD4 count & WNL & $<\mathrm{LLN}-500 / \mathrm{mm}^{3}$ & $200-<500 / \mathrm{mm}^{3}$ & $50-<200 / \mathrm{mm}^{3}$ & $<50 / \mathrm{mm}^{3}$ \\
\hline Haptoglobin & normal & decreased & - & absent & - \\
\hline Hemoglobin (Hgb) & WNL & $\begin{array}{l}<\mathrm{LLN}-10.0 \mathrm{~g} / \mathrm{dL} \\
<\mathrm{LLN}-100 \mathrm{~g} / \mathrm{L} \\
<\mathrm{LLN}-6.2 \mathrm{mmol} / \mathrm{L}\end{array}$ & $\begin{array}{l}8.0-<10.0 \mathrm{~g} / \mathrm{dL} \\
80-<100 \mathrm{~g} / \mathrm{L} \\
4.9-<6.2 \mathrm{mmol} / \mathrm{L}\end{array}$ & $\begin{array}{l}6.5-<8.0 \mathrm{~g} / \mathrm{dL} \\
65-<80 \mathrm{~g} / \mathrm{L} \\
4.0-<4.9 \mathrm{mmol} / \mathrm{L}\end{array}$ & $\begin{array}{l}<6.5 \mathrm{~g} / \mathrm{dL} \\
<65 \mathrm{~g} / \mathrm{L} \\
<4.0 \mathrm{mmol} / \mathrm{L}\end{array}$ \\
\hline $\begin{array}{l}\text { For leukemia studies or bone } \\
\text { marrow infiltrative/ } \\
\text { myelophthisic processes, if } \\
\text { specified in the protocol. }\end{array}$ & WNL & $\begin{array}{l}10-<25 \% \text { decrease } \\
\text { from pretreatment }\end{array}$ & $\begin{array}{l}25-<50 \% \text { decrease } \\
\text { from pretreatment }\end{array}$ & $\begin{array}{l}50-<75 \% \text { decrease } \\
\text { from pretreatment }\end{array}$ & $\begin{array}{l}\geq 75 \% \text { decrease from } \\
\text { pretreatment }\end{array}$ \\
\hline $\begin{array}{l}\text { Hemolysis (e.g., immune } \\
\text { hemolytic anemia, drug- } \\
\text { related hemolysis, other) }\end{array}$ & none & $\begin{array}{l}\text { only laboratory } \\
\text { evidence of hemolysis } \\
\text { [e.g., direct antiglobulin } \\
\text { test (DAT, Coombs') } \\
\text { schistocytes] }\end{array}$ & $\begin{array}{l}\text { evidence of red cell } \\
\text { destruction and } \geq 2 \mathrm{gm} \\
\text { decrease in hemoglobin, } \\
\text { no transfusion }\end{array}$ & $\begin{array}{l}\text { requiring transfusion } \\
\text { and/or medical } \\
\text { intervention (e.g., } \\
\text { steroids) }\end{array}$ & $\begin{array}{l}\text { catastrophic } \\
\text { consequences of } \\
\text { hemolysis (e.g., renal } \\
\text { failure, hypotension, } \\
\text { bronchospasm, } \\
\text { emergency } \\
\text { splenectomy) }\end{array}$ \\
\hline
\end{tabular}


CTC Version 2.0

Publish Date: April 30, 1999

\begin{tabular}{|c|c|c|c|c|c|}
\hline \multicolumn{6}{|c|}{ Grade } \\
\hline Adverse Event & $\mathbf{0}$ & 1 & 2 & 3 & 4 \\
\hline Leukocytes (total WBC) & WNL & $\begin{array}{l}<\mathrm{LLN}-3.0 \times 10^{9} / \mathrm{L} \\
<\mathrm{LLN}-3000 / \mathrm{mm}^{3}\end{array}$ & $\begin{array}{l}\geq 2.0-<3.0 \times 10^{9} / \mathrm{L} \\
\geq 2000-<3000 / \mathrm{mm}^{3}\end{array}$ & $\begin{array}{l}\geq 1.0-<2.0 \times 10^{9} / \mathrm{L} \\
\geq 1000-<2000 / \mathrm{mm}^{3}\end{array}$ & $\begin{array}{l}<1.0 \times 10^{9} / \mathrm{L} \\
<1000 / \mathrm{mm}^{3}\end{array}$ \\
\hline $\begin{array}{l}\text { For BMT studies, if } \\
\text { specified in the protocol. }\end{array}$ & WNL & $\begin{array}{l}\geq 2.0-<3.0 \times 10^{9} / \mathrm{L} \\
\geq 2000-<3000 / \mathrm{mm}^{3}\end{array}$ & $\begin{array}{l}\geq 1.0-<2.0 \times 10^{9} / \mathrm{L} \\
\geq 1000-<2000 / \mathrm{mm}^{3}\end{array}$ & $\begin{array}{l}\geq 0.5-<1.0 \times 10^{9} / \mathrm{L} \\
\geq 500-<1000 / \mathrm{mm}^{3}\end{array}$ & $\begin{array}{l}<0.5 \times 10^{9} / \mathrm{L} \\
<500 / \mathrm{mm}^{3}\end{array}$ \\
\hline $\begin{array}{l}\text { For pediatric BMT studies } \\
\text { (using age, race and sex } \\
\text { normal values), if specified } \\
\text { in the protocol. }\end{array}$ & & $\geq 75-<100 \%$ LLN & $\geq 50-<75 \%$ LLN & $\geq 25-50 \%$ LLN & $<25 \% L L N$ \\
\hline Lymphopenia & WNL & $\begin{array}{l}<\mathrm{LLN}-1.0 \times 10^{9} / \mathrm{L} \\
<\mathrm{LLN}-1000 / \mathrm{mm}^{3}\end{array}$ & $\begin{array}{l}\geq 0.5-<1.0 \times 10^{9} / \mathrm{L} \\
\geq 500-<1000 / \mathrm{mm}^{3}\end{array}$ & $\begin{array}{l}<0.5 \times 10^{9} / \mathrm{L} \\
<500 / \mathrm{mm}^{3}\end{array}$ & - \\
\hline $\begin{array}{l}\text { For pediatric BMT studies } \\
\text { (using age, race and sex } \\
\text { normal values), if specified } \\
\text { in the protocol. }\end{array}$ & & $\geq 75-<100 \% L L N$ & $\geq 50-<75 \% L L N$ & $\geq 25-<50 \% L L N$ & $<25 \% L L N$ \\
\hline $\begin{array}{l}\text { Neutrophils/granulocytes } \\
\text { (ANC/AGC) }\end{array}$ & WNL & $\begin{array}{l}\geq 1.5-<2.0 \times 10^{9} / \mathrm{L} \\
\geq 1500-<2000 / \mathrm{mm}^{3}\end{array}$ & $\begin{array}{l}\geq 1.0-<1.5 \times 10^{9} / \mathrm{L} \\
\geq 1000-<1500 / \mathrm{mm}^{3}\end{array}$ & $\begin{array}{l}\geq 0.5-<1.0 \times 10^{9} / \mathrm{L} \\
\geq 500-<1000 / \mathrm{mm}^{3}\end{array}$ & $\begin{array}{l}<0.5 \times 10^{9} / \mathrm{L} \\
<500 / \mathrm{mm}^{3}\end{array}$ \\
\hline $\begin{array}{l}\text { For BMT studies, if } \\
\text { specified in the protocol. }\end{array}$ & WNL & $\begin{array}{l}\geq 1.0-<1.5 \times 10^{9} / \mathrm{L} \\
\geq 1000-<1500 / \mathrm{mm}^{3}\end{array}$ & $\begin{array}{l}\geq 0.5-<1.0 \times 10^{9} / \mathrm{L} \\
\geq 500-<1000 / \mathrm{mm}^{3}\end{array}$ & $\begin{array}{l}\geq 0.1-<0.5 \times 10^{9} / \mathrm{L} \\
\geq 100-<500 / \mathrm{mm}^{3}\end{array}$ & $\begin{array}{l}<0.1 \times 10^{9} / \mathrm{L} \\
<100 / \mathrm{mm}^{3}\end{array}$ \\
\hline $\begin{array}{l}\text { For leukemia studies or bone } \\
\text { marrow infiltrative/ } \\
\text { myelophthisic process, if } \\
\text { specified in the protocol. }\end{array}$ & WNL & $\begin{array}{l}10-<25 \% \text { decrease } \\
\text { from baseline }\end{array}$ & $\begin{array}{l}25-<50 \% \text { decrease } \\
\text { from baseline }\end{array}$ & $\begin{array}{l}50-<75 \% \text { decrease } \\
\text { from baseline }\end{array}$ & $\begin{array}{l}\geq 75 \% \text { decrease from } \\
\text { baseline }\end{array}$ \\
\hline Platelets & WNL & $\begin{array}{l}<\mathrm{LLN}-75.0 \times 10^{9} / \mathrm{L} \\
<\mathrm{LLN}-75,000 / \mathrm{mm}^{3}\end{array}$ & $\begin{array}{l}\geq 50.0-<75.0 \times 10^{9} / \mathrm{L} \\
\geq 50,000-<75,000 / \mathrm{mm}^{3}\end{array}$ & $\begin{array}{l}\geq 10.0-<50.0 \times 10^{9} / \mathrm{L} \\
\geq 10,000-<50,000 / \mathrm{mm}^{3}\end{array}$ & $\begin{array}{l}<10.0 \times 10^{9} / \mathrm{L} \\
<10,000 / \mathrm{mm}^{3}\end{array}$ \\
\hline $\begin{array}{l}\text { For BMT studies, if } \\
\text { specified in the protocol. }\end{array}$ & WNL & $\begin{array}{l}\geq 50.0-<75.0 \times 10^{9} / \mathrm{L} \\
\geq 50,000-<75,000 / \mathrm{mm}^{3}\end{array}$ & $\begin{array}{l}\geq 20.0-<50.0 \times 10^{9} / \mathrm{L} \\
\geq 20,000-<50,000 / \mathrm{mm}^{3}\end{array}$ & $\begin{array}{l}\geq 10.0-<20.0 \times 10^{9} / \mathrm{L} \\
\geq 10,000-<20,000 / \mathrm{mm}^{3}\end{array}$ & $\begin{array}{l}<10.0 \times 10^{9} / \mathrm{L} \\
<10,000 / \mathrm{mm}^{3}\end{array}$ \\
\hline $\begin{array}{l}\text { For leukemia studies or bone } \\
\text { marrow infiltrative/ } \\
\text { myelophthisic process, if } \\
\text { specified in the protocol. }\end{array}$ & WNL & $\begin{array}{l}10-<25 \% \text { decrease } \\
\text { from baseline }\end{array}$ & $\begin{array}{l}25-<50 \% \text { decrease } \\
\text { from baseline }\end{array}$ & $\begin{array}{l}50-<75 \% \text { decrease } \\
\text { from baseline }\end{array}$ & $\begin{array}{l}\geq 75 \% \text { decrease from } \\
\text { baseline }\end{array}$ \\
\hline Transfusion: Platelets & none & - & - & yes & $\begin{array}{l}\text { platelet transfusions and } \\
\text { other measures required } \\
\text { to improve platelet } \\
\text { increment; platelet } \\
\text { transfusion } \\
\text { refractoriness associated } \\
\text { with life-threatening } \\
\text { bleeding. (e.g., HLA or } \\
\text { cross matched platelet } \\
\text { transfusions) }\end{array}$ \\
\hline $\begin{array}{l}\text { For BMT studies, if } \\
\text { specified in the protocol. }\end{array}$ & none & $\begin{array}{l}1 \text { platelet transfusion in } \\
24 \text { hours }\end{array}$ & $\begin{array}{l}2 \text { platelet transfusions in } \\
24 \text { hours }\end{array}$ & $\begin{array}{l}\geq 3 \text { platelet transfusions } \\
\text { in } 24 \text { hours }\end{array}$ & $\begin{array}{l}\text { platelet transfusions and } \\
\text { other measures required } \\
\text { to improve platelet } \\
\text { increment; platelet } \\
\text { transfusion } \\
\text { refractoriness associated } \\
\text { with life-threatening } \\
\text { bleeding. (e.g., HLA or } \\
\text { cross matched platelet } \\
\text { transfusions) }\end{array}$ \\
\hline
\end{tabular}


CTC Version 2.0

Publish Date: April 30, 1999

\begin{tabular}{|c|c|c|c|c|c|}
\hline \multicolumn{6}{|c|}{ Grade } \\
\hline Adverse Event & $\mathbf{0}$ & $\mathbf{1}$ & 2 & 3 & 4 \\
\hline Transfusion: pRBCs & none & - & - & yes & - \\
\hline $\begin{array}{l}\text { For BMT studies, if } \\
\text { specified in the protocol. }\end{array}$ & none & $\begin{array}{l}\leq 2 \mathrm{u} \text { pRBC in } 24 \text { hours } \\
\text { elective or planned }\end{array}$ & $\begin{array}{l}3 \text { u pRBC in } 24 \text { hours } \\
\text { elective or planned }\end{array}$ & $\geq 4 \mathrm{u}$ pRBC in 24 hours & $\begin{array}{l}\text { hemorrhage or } \\
\text { hemolysis associated } \\
\text { with life-threatening } \\
\text { anemia; medical } \\
\text { intervention required to } \\
\text { improve hemoglobin }\end{array}$ \\
\hline $\begin{array}{l}\text { For pediatric BMT studies, if } \\
\text { specified in the protocol. }\end{array}$ & none & $\begin{array}{l}\leq 15 \mathrm{~mL} / \mathrm{kg} \text { in } 24 \text { hours } \\
\text { elective or planned }\end{array}$ & $\begin{array}{l}>15-\leq 30 \mathrm{~mL} / \mathrm{kg} \text { in } 24 \\
\text { hours elective or } \\
\text { planned }\end{array}$ & $>30 \mathrm{~mL} / \mathrm{kg}$ in 24 hours & $\begin{array}{l}\text { hemorrhage or } \\
\text { hemolysis associated } \\
\text { with life-threatening } \\
\text { anemia; medical } \\
\text { intervention required to } \\
\text { improve hemoglobin }\end{array}$ \\
\hline \multicolumn{6}{|l|}{ Also consider Hemoglobin. } \\
\hline $\begin{array}{l}\text { Blood/Bone Marrow - Other } \\
\text { (Specify, }\end{array}$ & none & mild & moderate & severe & $\begin{array}{l}\text { life-threatening or } \\
\text { disabling }\end{array}$ \\
\hline \multicolumn{6}{|c|}{ CARDIOVASCULAR (ARRHYTHMIA) } \\
\hline $\begin{array}{l}\text { Conduction abnormality/ } \\
\text { Atrioventricular heart block }\end{array}$ & none & $\begin{array}{l}\text { asymptomatic, not } \\
\text { requiring treatment } \\
\text { (e.g., Mobitz type I } \\
\text { second-degree AV } \\
\text { block, Wenckebach) }\end{array}$ & $\begin{array}{l}\text { symptomatic, but not } \\
\text { requiring treatment }\end{array}$ & $\begin{array}{l}\text { symptomatic and } \\
\text { requiring treatment } \\
\text { (e.g., Mobitz type II } \\
\text { second-degree AV } \\
\text { block, third-degree AV } \\
\text { block) }\end{array}$ & $\begin{array}{l}\text { life-threatening (e.g., } \\
\text { arrhythmia associated } \\
\text { with CHF, hypotension, } \\
\text { syncope, shock) }\end{array}$ \\
\hline $\begin{array}{l}\text { Nodal/junctional } \\
\text { arrhythmia/dysrhythmia }\end{array}$ & none & $\begin{array}{l}\text { asymptomatic, not } \\
\text { requiring treatment }\end{array}$ & $\begin{array}{l}\text { symptomatic, but not } \\
\text { requiring treatment }\end{array}$ & $\begin{array}{l}\text { symptomatic and } \\
\text { requiring treatment }\end{array}$ & $\begin{array}{l}\text { life-threatening (e.g., } \\
\text { arrhythmia associated } \\
\text { with CHF, hypotension, } \\
\text { syncope, shock) }\end{array}$ \\
\hline Palpitations & none & present & - & - & - \\
\hline $\begin{array}{l}\text { Prolonged QTc interval } \\
\text { (QTc }>0.48 \text { seconds) }\end{array}$ & none & $\begin{array}{l}\text { asymptomatic, not } \\
\text { requiring treatment }\end{array}$ & $\begin{array}{l}\text { symptomatic, but not } \\
\text { requiring treatment }\end{array}$ & $\begin{array}{l}\text { symptomatic and } \\
\text { requiring treatment }\end{array}$ & $\begin{array}{l}\text { life-threatening (e.g., } \\
\text { arrhythmia associated } \\
\text { with CHF, hypotension, } \\
\text { syncope, shock) }\end{array}$ \\
\hline Sinus bradycardia & none & $\begin{array}{l}\text { asymptomatic, not } \\
\text { requiring treatment }\end{array}$ & $\begin{array}{l}\text { symptomatic, but not } \\
\text { requiring treatment }\end{array}$ & $\begin{array}{l}\text { symptomatic and } \\
\text { requiring treatment }\end{array}$ & $\begin{array}{l}\text { life-threatening (e.g., } \\
\text { arrhythmia associated } \\
\text { with CHF, hypotension, } \\
\text { syncope, shock) }\end{array}$ \\
\hline Sinus tachycardia & none & $\begin{array}{l}\text { asymptomatic, not } \\
\text { requiring treatment }\end{array}$ & $\begin{array}{l}\text { symptomatic, but not } \\
\text { requiring treatment }\end{array}$ & $\begin{array}{l}\text { symptomatic and } \\
\text { requiring treatment of } \\
\text { underlying cause }\end{array}$ & - \\
\hline $\begin{array}{l}\text { Supraventricular arrhythmias } \\
\text { (SVT/atrial fibrillation/ } \\
\text { flutter) }\end{array}$ & none & $\begin{array}{l}\text { asymptomatic, not } \\
\text { requiring treatment }\end{array}$ & $\begin{array}{l}\text { symptomatic, but not } \\
\text { requiring treatment }\end{array}$ & $\begin{array}{l}\text { symptomatic and } \\
\text { requiring treatment }\end{array}$ & $\begin{array}{l}\text { life-threatening (e.g., } \\
\text { arrhythmia associated } \\
\text { with CHF, hypotension, } \\
\text { syncope, shock) }\end{array}$ \\
\hline \multicolumn{6}{|c|}{ Syncope (fainting) is graded in the NEUROLOGY category. } \\
\hline Vasovagal episode & none & - & $\begin{array}{l}\text { present without loss of } \\
\text { consciousness }\end{array}$ & $\begin{array}{l}\text { present with loss of } \\
\text { consciousness }\end{array}$ & - \\
\hline
\end{tabular}


CTC Version 2.0

Publish Date: April 30, 1999

\begin{tabular}{|c|c|c|c|c|c|}
\hline \multicolumn{6}{|c|}{ Grade } \\
\hline Adverse Event & $\mathbf{0}$ & 1 & 2 & 3 & 4 \\
\hline $\begin{array}{l}\text { Ventricular arrhythmia } \\
\text { (PVCs/bigeminy/trigeminy/ } \\
\text { ventricular tachycardia) }\end{array}$ & none & $\begin{array}{l}\text { asymptomatic, not } \\
\text { requiring treatment }\end{array}$ & $\begin{array}{l}\text { symptomatic, but not } \\
\text { requiring treatment }\end{array}$ & $\begin{array}{l}\text { symptomatic and } \\
\text { requiring treatment }\end{array}$ & $\begin{array}{l}\text { life-threatening (e.g., } \\
\text { arrhythmia associated } \\
\text { with CHF, hypotension, } \\
\text { syncope, shock) }\end{array}$ \\
\hline $\begin{array}{l}\text { Cardiovascular/ } \\
\text { Arrhythmia - Other } \\
\text { (Specify, }\end{array}$ & none & $\begin{array}{l}\text { asymptomatic, not } \\
\text { requiring treatment }\end{array}$ & $\begin{array}{l}\text { symptomatic, but not } \\
\text { requiring treatment }\end{array}$ & $\begin{array}{l}\text { symptomatic, and } \\
\text { requiring treatment of } \\
\text { underlying cause }\end{array}$ & $\begin{array}{l}\text { life-threatening (e.g., } \\
\text { arrhythmia associated } \\
\text { with CHF, hypotension, } \\
\text { syncope, shock) }\end{array}$ \\
\hline \multicolumn{6}{|c|}{ CARDIOVASCULAR (GENERAL) } \\
\hline $\begin{array}{l}\text { Acute vascular leak } \\
\text { syndrome }\end{array}$ & absent & - & $\begin{array}{l}\text { symptomatic, but not } \\
\text { requiring fluid support }\end{array}$ & $\begin{array}{l}\text { respiratory compromise } \\
\text { or requiring fluids }\end{array}$ & $\begin{array}{l}\text { life-threatening; } \\
\text { requiring pressor } \\
\text { support and/or } \\
\text { ventilatory support }\end{array}$ \\
\hline Cardiac-ischemia/infarction & none & $\begin{array}{l}\text { non-specific } \mathrm{T} \text { - wave } \\
\text { flattening or changes }\end{array}$ & $\begin{array}{l}\text { asymptomatic, } \mathrm{ST} \text { - and } \\
\mathrm{T} \text { - wave changes } \\
\text { suggesting ischemia }\end{array}$ & $\begin{array}{l}\text { angina without evidence } \\
\text { of infarction }\end{array}$ & $\begin{array}{l}\text { acute myocardial } \\
\text { infarction }\end{array}$ \\
\hline $\begin{array}{l}\text { Cardiac left ventricular } \\
\text { function }\end{array}$ & normal & $\begin{array}{l}\text { asymptomatic decline } \\
\text { of resting ejection } \\
\text { fraction of } \geq 10 \% \text { but } \\
<20 \% \text { of baseline value; } \\
\text { shortening fraction } \\
\geq 24 \% \text { but }<30 \%\end{array}$ & $\begin{array}{l}\text { asymptomatic but } \\
\text { resting ejection fraction } \\
\text { below LLN for } \\
\text { laboratory or decline of } \\
\text { resting ejection fraction } \\
\geq 20 \% \text { of baseline value; } \\
<24 \% \text { shortening } \\
\text { fraction }\end{array}$ & $\begin{array}{l}\text { CHF responsive to } \\
\text { treatment }\end{array}$ & $\begin{array}{l}\text { severe or refractory } \\
\text { CHF or requiring } \\
\text { intubation }\end{array}$ \\
\hline \multicolumn{6}{|c|}{ CNS cerebrovascular ischemia is graded in the NEUROLOGY category. } \\
\hline Cardiac troponin I (cTnI) & normal & - & - & $\begin{array}{l}\text { levels consistent with } \\
\text { unstable angina as } \\
\text { defined by the } \\
\text { manufacturer }\end{array}$ & $\begin{array}{l}\text { levels consistent with } \\
\text { myocardial infarction as } \\
\text { defined by the } \\
\text { manufacturer }\end{array}$ \\
\hline Cardiac troponin $\mathrm{T}(\mathrm{cTnT})$ & normal & $\geq 0.03-<0.05 \mathrm{ng} / \mathrm{mL}$ & $\geq 0.05-<0.1 \mathrm{ng} / \mathrm{mL}$ & $\geq 0.1-<0.2 \mathrm{ng} / \mathrm{mL}$ & $\geq 0.2 \mathrm{ng} / \mathrm{mL}$ \\
\hline Edema & none & $\begin{array}{l}\text { asymptomatic, not } \\
\text { requiring therapy }\end{array}$ & $\begin{array}{l}\text { symptomatic, requiring } \\
\text { therapy }\end{array}$ & $\begin{array}{l}\text { symptomatic edema } \\
\text { limiting function and } \\
\text { unresponsive to therapy } \\
\text { or requiring drug } \\
\text { discontinuation }\end{array}$ & $\begin{array}{l}\text { anasarca (severe } \\
\text { generalized edema) }\end{array}$ \\
\hline Hypertension & none & $\begin{array}{l}\text { asymptomatic, transient } \\
\text { increase by }>20 \mathrm{mmHg} \\
\text { (diastolic) or to } \\
>150 / 100 * \text { if previously } \\
\text { WNL; not requiring } \\
\text { treatment }\end{array}$ & $\begin{array}{l}\text { recurrent or persistent } \\
\text { or symptomatic increase } \\
\text { by }>20 \mathrm{mmHg} \\
\text { (diastolic) or to } \\
>150 / 100 * \text { if previously } \\
\text { WNL; not requiring } \\
\text { treatment }\end{array}$ & $\begin{array}{l}\text { requiring therapy or } \\
\text { more intensive therapy } \\
\text { than previously }\end{array}$ & hypertensive crisis \\
\hline
\end{tabular}


CTC Version 2.0

Publish Date: April 30, 1999

\begin{tabular}{|c|c|c|c|c|c|}
\hline \multicolumn{6}{|c|}{ Grade } \\
\hline Adverse Event & $\mathbf{0}$ & 1 & 2 & 3 & 4 \\
\hline Hypotension & none & $\begin{array}{l}\text { changes, but not } \\
\text { requiring therapy } \\
\text { (including transient } \\
\text { orthostatic hypotension) }\end{array}$ & $\begin{array}{l}\text { requiring brief fluid } \\
\text { replacement or other } \\
\text { therapy but not } \\
\text { hospitalization; no } \\
\text { physiologic } \\
\text { consequences }\end{array}$ & $\begin{array}{l}\text { requiring therapy and } \\
\text { sustained medical } \\
\text { attention, but resolves } \\
\text { without persisting } \\
\text { physiologic } \\
\text { consequences }\end{array}$ & $\begin{array}{l}\text { shock (associated with } \\
\text { acidemia and impairing } \\
\text { vital organ function due } \\
\text { to tissue hypoperfusion) }\end{array}$ \\
\hline \multicolumn{6}{|c|}{ Also consider Syncope (fainting). } \\
\hline \multirow{2}{*}{\multicolumn{6}{|c|}{$\begin{array}{l}\text { Notes: Angina or MI is graded as Cardiac-ischemia/infarction in the CARDIOVASCULAR (GENERAL) category. } \\
\text { For pediatric patients, systolic BP } 65 \mathrm{mmHg} \text { or less in infants up to } 1 \text { year old and } 70 \mathrm{mmHg} \text { or less in children older than } 1 \text { year of age, use two successive } \\
\text { or three measurements in } 24 \text { hours. }\end{array}$}} \\
\hline & & & & & \\
\hline Myocarditis & none & - & - & $\begin{array}{l}\text { CHF responsive to } \\
\text { treatment }\end{array}$ & $\begin{array}{l}\text { severe or refractory } \\
\text { CHF }\end{array}$ \\
\hline $\begin{array}{l}\text { Operative injury of } \\
\text { vein/artery }\end{array}$ & none & $\begin{array}{l}\text { primary suture repair } \\
\text { for injury, but not } \\
\text { requiring transfusion }\end{array}$ & $\begin{array}{l}\text { primary suture repair } \\
\text { for injury, requiring } \\
\text { transfusion }\end{array}$ & $\begin{array}{l}\text { vascular occlusion } \\
\text { requiring surgery or } \\
\text { bypass for injury }\end{array}$ & $\begin{array}{l}\text { myocardial infarction; } \\
\text { resection of organ (e.g., } \\
\text { bowel, limb) }\end{array}$ \\
\hline $\begin{array}{l}\text { Pericardial effusion/ } \\
\text { pericarditis }\end{array}$ & none & $\begin{array}{l}\text { asymptomatic effusion, } \\
\text { not requiring treatment }\end{array}$ & $\begin{array}{l}\text { pericarditis (rub, ECG } \\
\text { changes, and/or chest } \\
\text { pain) }\end{array}$ & $\begin{array}{l}\text { with physiologic } \\
\text { consequences }\end{array}$ & $\begin{array}{l}\text { tamponade (drainage or } \\
\text { pericardial window } \\
\text { required) }\end{array}$ \\
\hline Peripheral arterial ischemia & none & - & $\begin{array}{l}\text { brief episode of } \\
\text { ischemia managed non- } \\
\text { surgically and without } \\
\text { permanent deficit }\end{array}$ & $\begin{array}{l}\text { requiring surgical } \\
\text { intervention }\end{array}$ & $\begin{array}{l}\text { life-threatening or with } \\
\text { permanent functional } \\
\text { deficit (e.g., } \\
\text { amputation) }\end{array}$ \\
\hline Phlebitis (superficial) & none & - & present & - & - \\
\hline \multicolumn{6}{|c|}{ Notes: Injection site reaction is graded in the DERMATOLOGY/SKIN category. } \\
\hline \multicolumn{6}{|c|}{ Thrombosis/embolism is graded in the CARDIOVASCULAR (GENERAL) category. } \\
\hline \multicolumn{6}{|c|}{ Syncope (fainting) is graded in the NEUROLOGY category. } \\
\hline Thrombosis/embolism & none & - & $\begin{array}{l}\text { deep vein thrombosis, } \\
\text { not requiring } \\
\text { anticoagulant }\end{array}$ & $\begin{array}{l}\text { deep vein thrombosis, } \\
\text { requiring anticoagulant } \\
\text { therapy }\end{array}$ & $\begin{array}{l}\text { embolic event including } \\
\text { pulmonary embolism }\end{array}$ \\
\hline \multicolumn{6}{|c|}{ Vein/artery operative injury is graded as Operative injury of vein/artery in the CARDIOVASCULAR (GENERAL) category. } \\
\hline $\begin{array}{l}\text { Visceral arterial ischemia } \\
\text { (non-myocardial) }\end{array}$ & none & - & $\begin{array}{l}\text { brief episode of } \\
\text { ischemia managed non- } \\
\text { surgically and without } \\
\text { permanent deficit }\end{array}$ & $\begin{array}{l}\text { requiring surgical } \\
\text { intervention }\end{array}$ & $\begin{array}{l}\text { life-threatening or with } \\
\text { permanent functional } \\
\text { deficit (e.g., resection of } \\
\text { ileum) }\end{array}$ \\
\hline $\begin{array}{l}\text { Cardiovascular/ } \\
\text { General - Other } \\
\text { (Specify, }\end{array}$ & none & mild & moderate & severe & $\begin{array}{l}\text { life-threatening or } \\
\text { disabling }\end{array}$ \\
\hline
\end{tabular}


CTC Version 2.0

Publish Date: April 30, 1999

\begin{tabular}{|c|c|c|c|c|c|}
\hline \multicolumn{6}{|c|}{ Grade } \\
\hline Adverse Event & $\mathbf{0}$ & $\mathbf{1}$ & 2 & 3 & 4 \\
\hline \multicolumn{6}{|c|}{ COAGULATION } \\
\hline \multicolumn{6}{|c|}{ Note: See the HEMORRHAGE category for grading the severity of bleeding events. } \\
\hline $\begin{array}{l}\text { DIC } \\
\text { (disseminated intravascular } \\
\text { coagulation) }\end{array}$ & absent & - & - & $\begin{array}{l}\text { laboratory findings } \\
\text { present with no } \\
\text { bleeding }\end{array}$ & $\begin{array}{l}\text { laboratory findings and } \\
\text { bleeding }\end{array}$ \\
\hline \multicolumn{6}{|l|}{ Also consider Platelets. } \\
\hline \multicolumn{6}{|c|}{ Note: Must have increased fibrin split products or D-dimer in order to grade as DIC. } \\
\hline Fibrinogen & WNL & $\geq 0.75-<1.0 \times$ LLN & $\geq 0.5-<0.75 \times$ LLN & $\geq 0.25-<0.5 \times$ LLN & $<0.25 \times \operatorname{LLN}$ \\
\hline $\begin{array}{l}\text { For leukemia studies or bone } \\
\text { marrow infiltrative/ } \\
\text { myelophthisic process, if } \\
\text { specified in the protocol. }\end{array}$ & WNL & $\begin{array}{l}<20 \% \text { decrease from } \\
\text { pretreatment value or } \\
\text { LLN }\end{array}$ & $\begin{array}{l}\geq 20-<40 \% \text { decrease } \\
\text { from pretreatment value } \\
\text { or LLN }\end{array}$ & $\begin{array}{l}\geq 40-<70 \% \text { decrease } \\
\text { from pretreatment value } \\
\text { or LLN }\end{array}$ & $<50 \mathrm{mg}$ \\
\hline $\begin{array}{l}\text { Partial thromboplastin time } \\
\text { (PTT) }\end{array}$ & WNL & $>\mathrm{ULN}-\leq 1.5 \times \mathrm{ULN}$ & $>1.5-\leq 2 \times$ ULN & $>2 \times \mathrm{ULN}$ & - \\
\hline \multicolumn{6}{|c|}{ Phlebitis is graded in the CARDIOVASCULAR (GENERAL) category. } \\
\hline Prothrombin time (PT) & WNL & $>\mathrm{ULN}-\leq 1.5 \times \mathrm{ULN}$ & $>1.5-\leq 2 \times$ ULN & $>2 \times \mathrm{ULN}$ & - \\
\hline \multicolumn{6}{|c|}{ Thrombosis/embolism is graded in the CARDIOVASCULAR (GENERAL) category. } \\
\hline $\begin{array}{l}\text { Thrombotic } \\
\text { microangiopathy (e.g., } \\
\text { thrombotic } \\
\text { thrombocytopenic } \\
\text { purpura/TTP or hemolytic } \\
\text { uremic syndrome/HUS) }\end{array}$ & absent & - & - & $\begin{array}{l}\text { laboratory findings } \\
\text { present without clinical } \\
\text { consequences }\end{array}$ & $\begin{array}{l}\text { laboratory findings and } \\
\text { clinical consequences, } \\
\text { (e.g., CNS hemorrhage/ } \\
\text { bleeding or thrombosis/ } \\
\text { embolism or renal } \\
\text { failure) requiring } \\
\text { therapeutic intervention }\end{array}$ \\
\hline $\begin{array}{l}\text { For BMT studies, if } \\
\text { specified in the protocol. }\end{array}$ & - & $\begin{array}{l}\text { evidence of RBC } \\
\text { destruction } \\
\text { (schistocytosis) without } \\
\text { clinical consequences }\end{array}$ & $\begin{array}{l}\text { evidence of RBC } \\
\text { destruction with } \\
\text { elevated creatinine }(\leq 3 \\
x \text { ULN) }\end{array}$ & $\begin{array}{l}\text { evidence of RBC } \\
\text { destruction with } \\
\text { creatinine }(>3 \mathrm{x} \text { ULN) } \\
\text { not requiring dialysis }\end{array}$ & $\begin{array}{l}\text { evidence of RBC } \\
\text { destruction with renal } \\
\text { failure requiring } \\
\text { dialysis and/or } \\
\text { encephalopathy }\end{array}$ \\
\hline \multicolumn{6}{|c|}{$\begin{array}{l}\text { Also consider Hemoglobin, Platelets, Creatinine. } \\
\text { Note: Must have microangiopathic changes on blood smear (e.g., schistocytes, helmet cells, red cell fragments). }\end{array}$} \\
\hline $\begin{array}{l}\text { Coagulation - Other } \\
\text { (Specify, }\end{array}$ & none & mild & moderate & severe & $\begin{array}{l}\text { life-threatening or } \\
\text { disabling }\end{array}$ \\
\hline \multicolumn{6}{|c|}{ CONSTITUTIONAL SYMPTOMS } \\
\hline $\begin{array}{l}\text { Fatigue } \\
\text { (lethargy, malaise, asthenia) }\end{array}$ & none & $\begin{array}{l}\text { increased fatigue over } \\
\text { baseline, but not } \\
\text { altering normal } \\
\text { activities }\end{array}$ & $\begin{array}{l}\text { moderate (e.g., decrease } \\
\text { in performance status } \\
\text { by } 1 \text { ECOG level or } \\
20 \% \text { Karnofsky or } \\
\text { Lansky) or causing } \\
\text { difficulty performing } \\
\text { some activities }\end{array}$ & $\begin{array}{l}\text { severe (e.g., decrease in } \\
\text { performance status by } \\
\geq 2 \text { ECOG levels or } 40 \% \\
\text { Karnofsky or Lansky) or } \\
\text { loss of ability to } \\
\text { perform some activities }\end{array}$ & bedridden or disabling \\
\hline
\end{tabular}


CTC Version 2.0

Publish Date: April 30, 1999

\begin{tabular}{|c|c|c|c|c|c|}
\hline \multicolumn{6}{|c|}{ Grade } \\
\hline Adverse Event & $\mathbf{0}$ & $\mathbf{1}$ & 2 & 3 & 4 \\
\hline $\begin{array}{l}\text { Fever (in the absence of } \\
\text { neutropenia, where } \\
\text { neutropenia is defined as } \\
\text { AGC }<1.0 \times 10^{9} / \mathrm{L} \text { ) }\end{array}$ & none & $\begin{array}{l}38.0-39.0^{\circ} \mathrm{C}(100.4- \\
\left.102.2^{\circ} \mathrm{F}\right)\end{array}$ & $\begin{array}{l}39.1-40.0^{\circ} \mathrm{C}(102.3- \\
\left.104.0^{\circ} \mathrm{F}\right)\end{array}$ & $\begin{array}{l}>40.0^{\circ} \mathrm{C}\left(>104.0^{\circ} \mathrm{F}\right) \text { for } \\
<24 \mathrm{hrs}\end{array}$ & $\begin{array}{l}>40.0^{\circ} \mathrm{C}\left(>104.0^{\circ} \mathrm{F}\right) \text { for } \\
>24 \mathrm{hrs}\end{array}$ \\
\hline \multicolumn{6}{|c|}{ Also consider Allergic reaction/hypersensitivity. } \\
\hline \multicolumn{6}{|c|}{ Note: The temperature measurements listed above are oral or tympanic. } \\
\hline \multicolumn{6}{|c|}{ Hot flashes/flushes are graded in the ENDOCRINE category. } \\
\hline Rigors, chills & none & $\begin{array}{l}\text { mild, requiring } \\
\text { symptomatic treatment } \\
\text { (e.g., blanket) or non- } \\
\text { narcotic medication }\end{array}$ & $\begin{array}{l}\text { severe and/or } \\
\text { prolonged, requiring } \\
\text { narcotic medication }\end{array}$ & $\begin{array}{l}\text { not responsive to } \\
\text { narcotic medication }\end{array}$ & - \\
\hline $\begin{array}{l}\text { Sweating } \\
\text { (diaphoresis) }\end{array}$ & normal & mild and occasional & frequent or drenching & - & - \\
\hline Weight gain & $<5 \%$ & $5-<10 \%$ & $10-<20 \%$ & $\geq 20 \%$ & - \\
\hline \multicolumn{6}{|c|}{ Also consider Ascites, Edema, Pleural effusion (non-malignant). } \\
\hline $\begin{array}{l}\text { Weight gain associated with } \\
\text { Veno-Occlusive Disease } \\
\text { (VOD) for BMT studies, if } \\
\text { specified in the protocol. }\end{array}$ & $<2 \%$ & $\geq 2-<5 \%$ & $\geq 5-<10 \%$ & $\geq 10 \%$ or as ascites & $\begin{array}{l}\geq 10 \% \text { or fluid retention } \\
\text { resulting in pulmonary } \\
\text { failure }\end{array}$ \\
\hline \multicolumn{6}{|c|}{ Also consider Ascites, Edema, Pleural effusion (non-malignant). } \\
\hline Weight loss & $<5 \%$ & $5-<10 \%$ & $10-<20 \%$ & $\geq 20 \%$ & - \\
\hline \multicolumn{6}{|c|}{ Also consider Vomiting, Dehydration, Diarrhea. } \\
\hline $\begin{array}{l}\text { Constitutional Symptoms - } \\
\text { Other } \\
\text { (Specify,____ }\end{array}$ & none & mild & moderate & severe & $\begin{array}{l}\text { life-threatening or } \\
\text { disabling }\end{array}$ \\
\hline \multicolumn{6}{|c|}{ DERMATOLOGY/SKIN } \\
\hline Alopecia & normal & mild hair loss & pronounced hair loss & - & - \\
\hline $\begin{array}{l}\text { Bruising } \\
\text { (in absence of grade } 3 \text { or } 4 \\
\text { thrombocytopenia) }\end{array}$ & none & $\begin{array}{l}\text { localized or in } \\
\text { dependent area }\end{array}$ & generalized & - & - \\
\hline \multicolumn{6}{|c|}{$\begin{array}{l}\text { Note: Bruising resulting from grade } 3 \text { or } 4 \text { thrombocytopenia is graded as Petechiae/purpura and Hemorrhage/bleeding with grade } 3 \text { or } 4 \text { thrombocytopenia in the } \\
\text { HEMORRHAGE category, not in the DERMATOLOGY/SKIN category. }\end{array}$} \\
\hline Dry skin & normal & $\begin{array}{l}\text { controlled with } \\
\text { emollients }\end{array}$ & $\begin{array}{l}\text { not controlled with } \\
\text { emollients }\end{array}$ & - & - \\
\hline $\begin{array}{l}\text { Erythema multiforme (e.g., } \\
\text { Stevens-Johnson syndrome, } \\
\text { toxic epidermal necrolysis) }\end{array}$ & absent & - & $\begin{array}{l}\text { scattered, but not } \\
\text { generalized eruption }\end{array}$ & $\begin{array}{l}\text { severe or requiring IV } \\
\text { fluids (e.g., generalized } \\
\text { rash or painful } \\
\text { stomatitis) }\end{array}$ & $\begin{array}{l}\text { life-threatening (e.g., } \\
\text { exfoliative or ulcerating } \\
\text { dermatitis or requiring } \\
\text { enteral or parenteral } \\
\text { nutritional support) }\end{array}$ \\
\hline Flushing & absent & present & - & - & - \\
\hline Hand-foot skin reaction & none & $\begin{array}{l}\text { skin changes or } \\
\text { dermatitis without pain } \\
\text { (e.g., erythema, peeling) }\end{array}$ & $\begin{array}{l}\text { skin changes with pain, } \\
\text { not interfering with } \\
\text { function }\end{array}$ & $\begin{array}{l}\text { skin changes with pain, } \\
\text { interfering with } \\
\text { function }\end{array}$ & - \\
\hline Injection site reaction & none & $\begin{array}{l}\text { pain or itching or } \\
\text { erythema }\end{array}$ & $\begin{array}{l}\text { pain or swelling, with } \\
\text { inflammation or } \\
\text { phlebitis }\end{array}$ & $\begin{array}{l}\text { ulceration or necrosis } \\
\text { that is severe or } \\
\text { prolonged, or requiring } \\
\text { surgery }\end{array}$ & - \\
\hline
\end{tabular}


CTC Version 2.0

Publish Date: April 30, 1999

\begin{tabular}{|c|c|c|c|c|c|}
\hline \multicolumn{6}{|c|}{ Grade } \\
\hline Adverse Event & $\mathbf{0}$ & $\mathbf{1}$ & 2 & 3 & 4 \\
\hline Nail changes & normal & $\begin{array}{l}\text { discoloration or ridging } \\
\text { (koilonychia) or pitting }\end{array}$ & $\begin{array}{l}\text { partial or complete loss } \\
\text { of nail(s) or pain in } \\
\text { nailbeds }\end{array}$ & - & - \\
\hline \multicolumn{6}{|c|}{ Petechiae is graded in the HEMORRHAGE category. } \\
\hline Photosensitivity & none & painless erythema & painful erythema & $\begin{array}{l}\text { erythema with } \\
\text { desquamation }\end{array}$ & - \\
\hline $\begin{array}{l}\text { Pigmentation changes (e.g., } \\
\text { vitiligo) }\end{array}$ & none & $\begin{array}{l}\text { localized pigmentation } \\
\text { changes }\end{array}$ & $\begin{array}{l}\text { generalized } \\
\text { pigmentation changes }\end{array}$ & - & - \\
\hline Pruritus & none & $\begin{array}{l}\text { mild or localized, } \\
\text { relieved spontaneously } \\
\text { or by local measures }\end{array}$ & $\begin{array}{l}\text { intense or widespread, } \\
\text { relieved spontaneously } \\
\text { or by systemic measures }\end{array}$ & $\begin{array}{l}\text { intense or widespread } \\
\text { and poorly controlled } \\
\text { despite treatment }\end{array}$ & - \\
\hline \multicolumn{6}{|c|}{ Purpura is graded in the HEMORRHAGE category. } \\
\hline Radiation dermatitis & none & $\begin{array}{l}\text { faint erythema or dry } \\
\text { desquamation }\end{array}$ & $\begin{array}{l}\text { moderate to brisk } \\
\text { erythema or a patchy } \\
\text { moist desquamation, } \\
\text { mostly confined to skin } \\
\text { folds and creases; } \\
\text { moderate edema }\end{array}$ & $\begin{array}{l}\text { confluent moist } \\
\text { desquamation } \geq 1.5 \mathrm{~cm} \\
\text { diameter and not } \\
\text { confined to skin folds; } \\
\text { pitting edema }\end{array}$ & $\begin{array}{l}\text { skin necrosis or } \\
\text { ulceration of full } \\
\text { thickness dermis; may } \\
\text { include bleeding not } \\
\text { induced by minor } \\
\text { trauma or abrasion }\end{array}$ \\
\hline \multicolumn{6}{|c|}{ Note: Pain associated with radiation dermatitis is graded separately in the PAIN category as Pain due to radiation. } \\
\hline $\begin{array}{l}\text { Radiation recall reaction } \\
\text { (reaction following } \\
\text { chemotherapy in the absence } \\
\text { of additional radiation } \\
\text { therapy that occurs in a } \\
\text { previous radiation port) }\end{array}$ & none & $\begin{array}{l}\text { faint erythema or dry } \\
\text { desquamation }\end{array}$ & $\begin{array}{l}\text { moderate to brisk } \\
\text { erythema or a patchy } \\
\text { moist desquamation, } \\
\text { mostly confined to skin } \\
\text { folds and creases; } \\
\text { moderate edema }\end{array}$ & $\begin{array}{l}\text { confluent moist } \\
\text { desquamation } \geq 1.5 \mathrm{~cm} \\
\text { diameter and not } \\
\text { confined to skin folds; } \\
\text { pitting edema }\end{array}$ & $\begin{array}{l}\text { skin necrosis or } \\
\text { ulceration of full } \\
\text { thickness dermis; may } \\
\text { include bleeding not } \\
\text { induced by minor } \\
\text { trauma or abrasion }\end{array}$ \\
\hline Rash/desquamation & none & $\begin{array}{l}\text { macular or papular } \\
\text { eruption or erythema } \\
\text { without associated } \\
\text { symptoms }\end{array}$ & $\begin{array}{l}\text { macular or papular } \\
\text { eruption or erythema } \\
\text { with pruritus or other } \\
\text { associated symptoms } \\
\text { covering <50\% of body } \\
\text { surface or localized } \\
\text { desquamation or other } \\
\text { lesions covering }<50 \% \\
\text { of body surface area }\end{array}$ & $\begin{array}{l}\text { symptomatic } \\
\text { generalized } \\
\text { erythroderma or } \\
\text { macular, papular or } \\
\text { vesicular eruption or } \\
\text { desquamation covering } \\
\geq 50 \% \text { of body surface } \\
\text { area }\end{array}$ & $\begin{array}{l}\text { generalized exfoliative } \\
\text { dermatitis or ulcerative } \\
\text { dermatitis }\end{array}$ \\
\hline \multicolumn{6}{|c|}{ Also consider Allergic reaction/hypersensitivity. } \\
\hline $\begin{array}{l}\text { Rash/dermatitis associated } \\
\text { with high-dose } \\
\text { chemotherapy or BMT } \\
\text { studies. }\end{array}$ & none & $\begin{array}{l}\text { faint erythema or dry } \\
\text { desquamation }\end{array}$ & $\begin{array}{l}\text { moderate to brisk } \\
\text { erythema or a patchy } \\
\text { moist desquamation, } \\
\text { mostly confined to skin } \\
\text { folds and creases; } \\
\text { moderate edema }\end{array}$ & $\begin{array}{l}\text { confluent moist } \\
\text { desquamation } \geq 1.5 \mathrm{~cm} \\
\text { diameter and not } \\
\text { confined to skin folds; } \\
\text { pitting edema }\end{array}$ & $\begin{array}{l}\text { skin necrosis or ulcera- } \\
\text { tion of full thickness } \\
\text { dermis; may include } \\
\text { spontaneous bleeding } \\
\text { not induced by minor } \\
\text { trauma or abrasion }\end{array}$ \\
\hline $\begin{array}{l}\text { Rash/desquamation } \\
\text { associated with graft versus } \\
\text { host disease (GVHD) for } \\
\text { BMT studies, if specified in } \\
\text { the protocol. }\end{array}$ & None & $\begin{array}{l}\text { macular or papular } \\
\text { eruption or erythema } \\
\text { covering }<25 \% \text { of body } \\
\text { surface area without } \\
\text { associated symptoms }\end{array}$ & $\begin{array}{l}\text { macular or papular } \\
\text { eruption or erythema } \\
\text { with pruritus or other } \\
\text { associated symptoms } \\
\text { covering } \geq 25 \text { - }<50 \% \text { of } \\
\text { body surface or } \\
\text { localized desquamation } \\
\text { or other lesions } \\
\text { covering } \geq 25 \text { - }<50 \% \text { of } \\
\text { body surface area }\end{array}$ & $\begin{array}{l}\text { symptomatic } \\
\text { generalized } \\
\text { erythroderma or } \\
\text { symptomatic macular, } \\
\text { papular or vesicular } \\
\text { eruption, with bullous } \\
\text { formation, or } \\
\text { desquamation covering } \\
\geq 50 \% \text { of body surface } \\
\text { area }\end{array}$ & $\begin{array}{l}\text { generalized exfoliative } \\
\text { dermatitis or ulcerative } \\
\text { dermatitis or bullous } \\
\text { formation }\end{array}$ \\
\hline A & er & & & & \\
\hline
\end{tabular}


CTC Version 2.0

Publish Date: April 30, 1999

\begin{tabular}{|c|c|c|c|c|c|}
\hline \multicolumn{6}{|c|}{ Grade } \\
\hline Adverse Event & $\mathbf{0}$ & 1 & 2 & 3 & 4 \\
\hline $\begin{array}{l}\text { Urticaria } \\
\text { (hives, welts, wheals) }\end{array}$ & none & requiring no medication & $\begin{array}{l}\text { requiring PO or topical } \\
\text { treatment or IV } \\
\text { medication or steroids } \\
\text { for }<24 \text { hours }\end{array}$ & $\begin{array}{l}\text { requiring IV medication } \\
\text { or steroids for } \geq 24 \\
\text { hours }\end{array}$ & - \\
\hline Wound-infectious & none & cellulitis & superficial infection & $\begin{array}{l}\text { infection requiring IV } \\
\text { antibiotics }\end{array}$ & necrotizing fasciitis \\
\hline Wound-non-infectious & none & incisional separation & incisional hernia & $\begin{array}{l}\text { fascial disruption } \\
\text { without evisceration }\end{array}$ & $\begin{array}{l}\text { fascial disruption with } \\
\text { evisceration }\end{array}$ \\
\hline $\begin{array}{l}\text { Dermatology/Skin - Other } \\
\text { (Specify, }\end{array}$ & none & mild & moderate & severe & $\begin{array}{l}\text { life-threatening or } \\
\text { disabling }\end{array}$ \\
\hline \multicolumn{6}{|c|}{ ENDOCRINE } \\
\hline $\begin{array}{l}\text { Cushingoid appearance (e.g., } \\
\text { moon face, buffalo hump, } \\
\text { centripetal obesity, } \\
\text { cutaneous striae) }\end{array}$ & absent & - & present & - & - \\
\hline \multicolumn{6}{|c|}{ Also consider Hyperglycemia, Hypokalemia. } \\
\hline Feminization of male & absent & - & - & present & - \\
\hline Gynecomastia & none & mild & pronounced or painful & $\begin{array}{l}\text { pronounced or painful } \\
\text { and requiring surgery }\end{array}$ & - \\
\hline Hot flashes/flushes & none & $\begin{array}{l}\text { mild or no more than } 1 \\
\text { per day }\end{array}$ & $\begin{array}{l}\text { moderate and greater } \\
\text { than } 1 \text { per day }\end{array}$ & - & - \\
\hline Hypothyroidism & absent & $\begin{array}{l}\text { asymptomatic,TSH } \\
\text { elevated, no therapy } \\
\text { given }\end{array}$ & $\begin{array}{l}\text { symptomatic or thyroid } \\
\text { replacement treatment } \\
\text { given }\end{array}$ & $\begin{array}{l}\text { patient hospitalized for } \\
\text { manifestations of } \\
\text { hypothyroidism }\end{array}$ & myxedema coma \\
\hline Masculinization of female & absent & - & - & present & - \\
\hline $\begin{array}{l}\text { SIADH (syndrome of } \\
\text { inappropriate antidiuretic } \\
\text { hormone) }\end{array}$ & absent & - & - & present & - \\
\hline $\begin{array}{l}\text { Endocrine - Other } \\
\text { (Specify, }\end{array}$ & none & mild & moderate & severe & $\begin{array}{l}\text { life-threatening or } \\
\text { disabling }\end{array}$ \\
\hline \multicolumn{6}{|c|}{ GASTROINTESTINAL } \\
\hline \multicolumn{6}{|c|}{ Amylase is graded in the METABOLIC/LABORATORY category. } \\
\hline Anorexia & none & loss of appetite & $\begin{array}{l}\text { oral intake significantly } \\
\text { decreased }\end{array}$ & requiring IV fluids & $\begin{array}{l}\text { requiring feeding tube } \\
\text { or parenteral nutrition }\end{array}$ \\
\hline Ascites (non-malignant) & none & asymptomatic & $\begin{array}{l}\text { symptomatic, requiring } \\
\text { diuretics }\end{array}$ & $\begin{array}{l}\text { symptomatic, requiring } \\
\text { therapeutic paracentesis }\end{array}$ & $\begin{array}{l}\text { life-threatening } \\
\text { physiologic } \\
\text { consequences }\end{array}$ \\
\hline Colitis & none & - & $\begin{array}{l}\text { abdominal pain with } \\
\text { mucus and/or blood in } \\
\text { stool }\end{array}$ & $\begin{array}{l}\text { abdominal pain, fever, } \\
\text { change in bowel habits } \\
\text { with ileus or peritoneal } \\
\text { signs, and radiographic } \\
\text { or biopsy } \\
\text { documentation }\end{array}$ & $\begin{array}{l}\text { perforation or requiring } \\
\text { surgery or toxic } \\
\text { megacolon }\end{array}$ \\
\hline \multicolumn{6}{|c|}{$\begin{array}{l}\text { Also consider Hemorrhage/bleeding with grade } 3 \text { or } 4 \text { thrombocytopenia, Hemorrhage/bleeding without grade } 3 \text { or } 4 \text { thrombocytopenia, Melena/GI bleeding, } \\
\text { Rectal bleeding/hematochezia, Hypotension. }\end{array}$} \\
\hline Constipation & none & $\begin{array}{l}\text { requiring stool softener } \\
\text { or dietary modification }\end{array}$ & requiring laxatives & $\begin{array}{l}\text { obstipation requiring } \\
\text { manual evacuation or } \\
\text { enema }\end{array}$ & $\begin{array}{l}\text { obstruction or toxic } \\
\text { megacolon }\end{array}$ \\
\hline
\end{tabular}


CTC Version 2.0

Publish Date: April 30, 1999

\begin{tabular}{|c|c|c|c|c|c|}
\hline \multicolumn{6}{|c|}{ Grade } \\
\hline Adverse Event & $\mathbf{0}$ & 1 & 2 & 3 & 4 \\
\hline Dehydration & none & $\begin{array}{l}\text { dry mucous membranes } \\
\text { and/or diminished skin } \\
\text { turgor }\end{array}$ & $\begin{array}{l}\text { requiring IV fluid } \\
\text { replacement (brief) }\end{array}$ & $\begin{array}{l}\text { requiring IV fluid } \\
\text { replacement (sustained) }\end{array}$ & $\begin{array}{l}\text { physiologic } \\
\text { consequences requiring } \\
\text { intensive care; } \\
\text { hemodynamic collapse }\end{array}$ \\
\hline \multicolumn{6}{|c|}{ Also consider Diarrhea, Vomiting, Stomatitis/pharyngitis (oral/pharyngeal mucositis), Hypotension. } \\
\hline $\begin{array}{l}\text { Diarrhea } \\
\text { patients without colostomy: }\end{array}$ & none & $\begin{array}{l}\text { increase of }<4 \\
\text { stools/day over pre- } \\
\text { treatment }\end{array}$ & $\begin{array}{l}\text { increase of } 4-6 \\
\text { stools/day, or nocturnal } \\
\text { stools }\end{array}$ & $\begin{array}{l}\text { increase of } \geq 7 \\
\text { stools/day or } \\
\text { incontinence; or need } \\
\text { for parenteral support } \\
\text { for dehydration }\end{array}$ & $\begin{array}{l}\text { physiologic } \\
\text { consequences requiring } \\
\text { intensive care; or } \\
\text { hemodynamic collapse }\end{array}$ \\
\hline patients with a colostomy: & none & $\begin{array}{l}\text { mild increase in loose, } \\
\text { watery colostomy } \\
\text { output compared with } \\
\text { pretreatment }\end{array}$ & $\begin{array}{l}\text { moderate increase in } \\
\text { loose, watery colostomy } \\
\text { output compared with } \\
\text { pretreatment, but not } \\
\text { interfering with normal } \\
\text { activity }\end{array}$ & $\begin{array}{l}\text { severe increase in loose, } \\
\text { watery colostomy } \\
\text { output compared with } \\
\text { pretreatment, interfering } \\
\text { with normal activity }\end{array}$ & $\begin{array}{l}\text { physiologic } \\
\text { consequences, requiring } \\
\text { intensive care; or } \\
\text { hemodynamic collapse }\end{array}$ \\
\hline $\begin{array}{l}\text { Diarrhea associated with } \\
\text { graft versus host disease } \\
\text { (GVHD) for BMT studies, if } \\
\text { specified in the protocol. }\end{array}$ & None & $\begin{array}{l}>500-\leq 1000 \mathrm{~mL} \text { of } \\
\text { diarrhea/day }\end{array}$ & $\begin{array}{l}>1000-\leq 1500 \mathrm{~mL} \text { of } \\
\text { diarrhea/day }\end{array}$ & $\begin{array}{l}>1500 \mathrm{~mL} \text { of } \\
\text { diarrhea/day }\end{array}$ & $\begin{array}{l}\text { severe abdominal pain } \\
\text { with or without ileus }\end{array}$ \\
\hline $\begin{array}{l}\text { For pediatric BMT studies, if } \\
\text { specified in the protocol. }\end{array}$ & & $\begin{array}{l}>5-\leq 10 \mathrm{~mL} / \mathrm{kg} \text { of } \\
\text { diarrhea/day }\end{array}$ & $\begin{array}{l}>10-\leq 15 \mathrm{~mL} / \mathrm{kg} \text { of } \\
\text { diarrhea/day }\end{array}$ & $\begin{array}{l}>15 \mathrm{~mL} / \mathrm{kg} \text { of } \\
\text { diarrhea/day }\end{array}$ & - \\
\hline \multicolumn{6}{|c|}{$\begin{array}{l}\text { Also consider Hemorrhage/bleeding with grade } 3 \text { or } 4 \text { thrombocytopenia, Hemorrhage/bleeding without grade } 3 \text { or } 4 \text { thrombocytopenia, Pain, Dehydration, } \\
\text { Hypotension. }\end{array}$} \\
\hline $\begin{array}{l}\text { Duodenal ulcer (requires } \\
\text { radiographic or endoscopic } \\
\text { documentation) }\end{array}$ & none & - & $\begin{array}{l}\text { requiring medical } \\
\text { management or non- } \\
\text { surgical treatment }\end{array}$ & $\begin{array}{l}\text { uncontrolled by } \\
\text { outpatient medical } \\
\text { management; requiring } \\
\text { hospitalization }\end{array}$ & $\begin{array}{l}\text { perforation or bleeding, } \\
\text { requiring emergency } \\
\text { surgery }\end{array}$ \\
\hline Dyspepsia/heartburn & none & mild & moderate & severe & - \\
\hline $\begin{array}{l}\text { Dysphagia, esophagitis, } \\
\text { odynophagia (painful } \\
\text { swallowing) }\end{array}$ & none & $\begin{array}{l}\text { mild dysphagia, but can } \\
\text { eat regular diet }\end{array}$ & $\begin{array}{l}\text { dysphagia, requiring } \\
\text { predominantly pureed, } \\
\text { soft, or liquid diet }\end{array}$ & $\begin{array}{l}\text { dysphagia, requiring IV } \\
\text { hydration }\end{array}$ & $\begin{array}{l}\text { complete obstruction } \\
\text { (cannot swallow saliva) } \\
\text { requiring enteral or } \\
\text { parenteral nutritional } \\
\text { support, or perforation }\end{array}$ \\
\hline \multicolumn{6}{|c|}{ Note: If the adverse event is radiation-related, grade either under Dysphagia-esophageal related to radiation or Dysphagia-pharyngeal related to radiation. } \\
\hline $\begin{array}{l}\text { Dysphagia-esophageal } \\
\text { related to radiation }\end{array}$ & none & $\begin{array}{l}\text { mild dysphagia, but can } \\
\text { eat regular diet }\end{array}$ & $\begin{array}{l}\text { dysphagia, requiring } \\
\text { predominantly pureed, } \\
\text { soft, or liquid diet }\end{array}$ & $\begin{array}{l}\text { Dysphagia, requiring } \\
\text { feeding tube, IV } \\
\text { hydration or } \\
\text { hyperalimentation }\end{array}$ & $\begin{array}{l}\text { complete obstruction } \\
\text { (cannot swallow saliva); } \\
\text { ulceration with bleeding } \\
\text { not induced by minor } \\
\text { trauma or abrasion or } \\
\text { perforation }\end{array}$ \\
\hline \multirow{2}{*}{\multicolumn{6}{|c|}{$\begin{array}{l}\text { Also consider Pain due to radiation, Mucositis due to radiation. } \\
\text { Note: Fistula is graded separately as Fistula-esophageal. }\end{array}$}} \\
\hline & & & & & \\
\hline $\begin{array}{l}\text { Dysphagia-pharyngeal } \\
\text { related to radiation }\end{array}$ & none & $\begin{array}{l}\text { mild dysphagia, but can } \\
\text { eat regular diet }\end{array}$ & $\begin{array}{l}\text { dysphagia, requiring } \\
\text { predominantly pureed, } \\
\text { soft, or liquid diet }\end{array}$ & $\begin{array}{l}\text { dysphagia, requiring } \\
\text { feeding tube, IV } \\
\text { hydration or } \\
\text { hyperalimentation }\end{array}$ & $\begin{array}{l}\text { complete obstruction } \\
\text { (cannot swallow saliva); } \\
\text { ulceration with bleeding } \\
\text { not induced by minor } \\
\text { trauma or abrasion or } \\
\text { perforation }\end{array}$ \\
\hline \multirow{2}{*}{\multicolumn{6}{|c|}{$\begin{array}{l}\text { Also consider Pain due to radiation, Mucositis due to radiation. } \\
\text { Note: Fistula is graded separately as Fistula-pharyngeal. }\end{array}$}} \\
\hline & & & & & \\
\hline Fistula-esophageal & none & - & - & present & requiring surgery \\
\hline Fistula-intestinal & none & - & - & present & requiring surgery \\
\hline
\end{tabular}


CTC Version 2.0

Publish Date: April 30, 1999

\begin{tabular}{|c|c|c|c|c|c|}
\hline \multicolumn{6}{|c|}{ Grade } \\
\hline Adverse Event & $\mathbf{0}$ & 1 & 2 & 3 & 4 \\
\hline Fistula-pharyngeal & none & - & - & present & requiring surgery \\
\hline Fistula-rectal/anal & none & - & - & present & requiring surgery \\
\hline Flatulence & none & mild & moderate & - & - \\
\hline $\begin{array}{l}\text { Gastric ulcer } \\
\text { (requires radiographic or } \\
\text { endoscopic documentation) }\end{array}$ & none & - & $\begin{array}{l}\text { requiring medical } \\
\text { management or non- } \\
\text { surgical treatment }\end{array}$ & $\begin{array}{l}\text { bleeding without } \\
\text { perforation, uncon- } \\
\text { trolled by outpatient } \\
\text { medical management; } \\
\text { requiring hospitalization } \\
\text { or surgery }\end{array}$ & $\begin{array}{l}\text { perforation or bleeding, } \\
\text { requiring emergency } \\
\text { surgery }\end{array}$ \\
\hline \multicolumn{6}{|c|}{ Also consider Hemorrhage/bleeding with grade 3 or 4 thrombocytopenia, Hemorrhage/bleeding without grade 3 or 4 thrombocytopenia. } \\
\hline Gastritis & none & - & $\begin{array}{l}\text { requiring medical } \\
\text { management or non- } \\
\text { surgical treatment }\end{array}$ & $\begin{array}{l}\text { uncontrolled by out- } \\
\text { patient medical } \\
\text { management; requiring } \\
\text { hospitalization or } \\
\text { surgery }\end{array}$ & $\begin{array}{l}\text { life-threatening } \\
\text { bleeding, requiring } \\
\text { emergency surgery }\end{array}$ \\
\hline \multicolumn{6}{|c|}{ Also consider Hemorrhage/bleeding with grade 3 or 4 thrombocytopenia, Hemorrhage/bleeding without grade 3 or 4 thrombocytopenia. } \\
\hline \multicolumn{6}{|c|}{ Hematemesis is graded in the HEMORRHAGE category. } \\
\hline \multicolumn{6}{|c|}{ Hematochezia is graded in the HEMORRHAGE category as Rectal bleeding/hematochezia. } \\
\hline Ileus (or neuroconstipation) & none & - & $\begin{array}{l}\text { intermittent, not } \\
\text { requiring intervention }\end{array}$ & $\begin{array}{l}\text { requiring non-surgical } \\
\text { intervention }\end{array}$ & requiring surgery \\
\hline Mouth dryness & normal & mild & moderate & - & - \\
\hline \multicolumn{6}{|l|}{ Mucositis } \\
\hline \multicolumn{6}{|c|}{$\begin{array}{l}\text { Notes: Mucositis not due to radiation is graded in the GASTROINTESTINAL category for specific sites: Colitis, Esophagitis, Gastritis, Stomatitis/pharyngitis } \\
\text { (oral/pharyngeal mucositis), and Typhlitis; or the RENAL/GENITOURINARY category for Vaginitis. }\end{array}$} \\
\hline \multicolumn{6}{|c|}{ Radiation-related mucositis is graded as Mucositis due to radiation. } \\
\hline Mucositis due to radiation & none & erythema of the mucosa & $\begin{array}{l}\text { patchy pseudomembra- } \\
\text { nous reaction (patches } \\
\text { generally } \leq 1.5 \mathrm{~cm} \text { in } \\
\text { diameter and non- } \\
\text { contiguous) }\end{array}$ & $\begin{array}{l}\text { confluent pseudomem- } \\
\text { branous reaction } \\
\text { (contiguous patches } \\
\text { generally }>1.5 \mathrm{~cm} \mathrm{in} \\
\text { diameter) }\end{array}$ & $\begin{array}{l}\text { necrosis or deep } \\
\text { ulceration; may include } \\
\text { bleeding not induced by } \\
\text { minor trauma or } \\
\text { abrasion }\end{array}$ \\
\hline \multicolumn{6}{|c|}{ Also consider Pain due to radiation. } \\
\hline \multicolumn{6}{|c|}{ Notes: Grade radiation mucositis of the larynx here. } \\
\hline \multicolumn{6}{|c|}{$\begin{array}{l}\text { Dysphagia related to radiation is also graded as either Dysphagia-esophageal related to radiation or Dysphagia-pharyngeal related to radiation, depending on } \\
\text { the site of treatment. }\end{array}$} \\
\hline Nausea & none & able to eat & $\begin{array}{l}\text { oral intake significantly } \\
\text { decreased }\end{array}$ & $\begin{array}{l}\text { no significant intake, } \\
\text { requiring IV fluids }\end{array}$ & - \\
\hline Pancreatitis & none & - & - & $\begin{array}{l}\text { abdominal pain with } \\
\text { pancreatic enzyme } \\
\text { elevation }\end{array}$ & $\begin{array}{l}\text { complicated by shock } \\
\text { (acute circulatory } \\
\text { failure) }\end{array}$ \\
\hline \multicolumn{6}{|l|}{ Also consider Hypotension. } \\
\hline Note: Amylase is graded in $\mathrm{t}$ & METAB & ATORY category. & & & \\
\hline
\end{tabular}


CTC Version 2.0

Publish Date: April 30, 1999

\begin{tabular}{|c|c|c|c|c|c|}
\hline \multicolumn{6}{|c|}{ Grade } \\
\hline Adverse Event & $\mathbf{0}$ & 1 & 2 & 3 & 4 \\
\hline Proctitis & none & $\begin{array}{l}\text { increased stool } \\
\text { frequency, occasional } \\
\text { blood-streaked stools or } \\
\text { rectal discomfort } \\
\text { (including hemorrhoids) } \\
\text { not requiring } \\
\text { medication }\end{array}$ & $\begin{array}{l}\text { increased stool } \\
\text { frequency, bleeding, } \\
\text { mucus discharge, or } \\
\text { rectal discomfort } \\
\text { requiring medication; } \\
\text { anal fissure }\end{array}$ & $\begin{array}{l}\text { increased stool fre- } \\
\text { quency/diarrhea requir- } \\
\text { ing parenteral support; } \\
\text { rectal bleeding requir- } \\
\text { ing transfusion; or per- } \\
\text { sistent mucus discharge, } \\
\text { necessitating pads }\end{array}$ & $\begin{array}{l}\text { perforation, bleeding or } \\
\text { necrosis or other life- } \\
\text { threatening } \\
\text { complication requiring } \\
\text { surgical intervention } \\
\text { (e.g., colostomy) }\end{array}$ \\
\hline \multicolumn{6}{|c|}{$\begin{array}{l}\text { Also consider Hemorrhage/bleeding with grade } 3 \text { or } 4 \text { thrombocytopenia, Hemorrhage/bleeding without grade } 3 \text { or } 4 \text { thrombocytopenia, Pain due to radiation. } \\
\text { Notes: Fistula is graded separately as Fistula-rectal/anal. }\end{array}$} \\
\hline \multicolumn{6}{|c|}{$\begin{array}{l}\text { Proctitis occurring more than } 90 \text { days after the start of radiation therapy is graded in the RTOG/EORTC Late Radiation Morbidity Scoring Scheme. (See } \\
\text { Appendix IV) }\end{array}$} \\
\hline Salivary gland changes & none & $\begin{array}{l}\text { slightly thickened } \\
\text { saliva; may have } \\
\text { slightly altered taste } \\
\text { (e.g., metallic); } \\
\text { additional fluids may be } \\
\text { required }\end{array}$ & $\begin{array}{l}\text { thick, ropy, sticky } \\
\text { saliva; markedly altered } \\
\text { taste; alteration in diet } \\
\text { required }\end{array}$ & - & $\begin{array}{l}\text { acute salivary gland } \\
\text { necrosis }\end{array}$ \\
\hline Sense of smell & normal & slightly altered & markedly altered & - & - \\
\hline $\begin{array}{l}\text { Stomatitis/pharyngitis } \\
\text { (oral/pharyngeal mucositis) }\end{array}$ & none & $\begin{array}{l}\text { painless ulcers, } \\
\text { erythema, or mild } \\
\text { soreness in the absence } \\
\text { of lesions }\end{array}$ & $\begin{array}{l}\text { painful erythema, } \\
\text { edema, or ulcers, but } \\
\text { can eat or swallow }\end{array}$ & $\begin{array}{l}\text { painful erythema, } \\
\text { edema, or ulcers } \\
\text { requiring IV hydration }\end{array}$ & $\begin{array}{l}\text { severe ulceration or } \\
\text { requires parenteral or } \\
\text { enteral nutritional } \\
\text { support or prophylactic } \\
\text { intubation }\end{array}$ \\
\hline $\begin{array}{l}\text { For BMT studies, if } \\
\text { specified in the protocol. }\end{array}$ & none & $\begin{array}{l}\text { painless ulcers, } \\
\text { erythema, or mild } \\
\text { soreness in the absence } \\
\text { of lesions }\end{array}$ & $\begin{array}{l}\text { painful erythema, } \\
\text { edema or ulcers but can } \\
\text { swallow }\end{array}$ & $\begin{array}{l}\text { painful erythema, } \\
\text { edema, or ulcers } \\
\text { preventing swallowing } \\
\text { or requiring hydration } \\
\text { or parenteral (or enteral) } \\
\text { nutritional support }\end{array}$ & $\begin{array}{l}\text { severe ulceration } \\
\text { requiring prophylactic } \\
\text { intubation or resulting } \\
\text { in documented } \\
\text { aspiration pneumonia }\end{array}$ \\
\hline \multicolumn{6}{|c|}{ Note: Radiation-related mucositis is graded as Mucositis due to radiation. } \\
\hline $\begin{array}{l}\text { Taste disturbance } \\
\text { (dysgeusia) }\end{array}$ & normal & slightly altered & markedly altered & - & - \\
\hline $\begin{array}{l}\text { Typhlitis } \\
\text { (inflammation of the cecum) }\end{array}$ & none & - & - & $\begin{array}{l}\text { abdominal pain, } \\
\text { diarrhea, fever, and } \\
\text { radiographic or biopsy } \\
\text { documentation }\end{array}$ & $\begin{array}{l}\text { perforation, bleeding or } \\
\text { necrosis or other life- } \\
\text { threatening } \\
\text { complication requiring } \\
\text { surgical intervention } \\
\text { (e.g., colostomy) }\end{array}$ \\
\hline \multicolumn{6}{|c|}{$\begin{array}{l}\text { Also consider Hemorrhage/bleeding with grade } 3 \text { or } 4 \text { thrombocytopenia, Hemorrhage/bleeding without grade } 3 \text { or } 4 \text { thrombocytopenia, Hypotension, Febrile } \\
\text { neutropenia. }\end{array}$} \\
\hline Vomiting & none & $\begin{array}{l}1 \text { episode in } 24 \text { hours } \\
\text { over pretreatment }\end{array}$ & $\begin{array}{l}2-5 \text { episodes in } 24 \text { hours } \\
\text { over pretreatment }\end{array}$ & $\begin{array}{l}\geq 6 \text { episodes in } 24 \text { hours } \\
\text { over pretreatment; or } \\
\text { need for IV fluids }\end{array}$ & $\begin{array}{l}\text { requiring parenteral } \\
\text { nutrition; or physiologic } \\
\text { consequences requiring } \\
\text { intensive care; } \\
\text { hemodynamic collapse }\end{array}$ \\
\hline \multicolumn{6}{|l|}{ Also consider Dehydration. } \\
\hline \multicolumn{6}{|c|}{ Weight gain is graded in the CONSTITUTIONAL SYMPTOMS category. } \\
\hline \multicolumn{6}{|c|}{ Weight loss is graded in the CONSTITUTIONAL SYMPTOMS category. } \\
\hline $\begin{array}{l}\text { Gastrointestinal - Other } \\
\text { (Specify, }\end{array}$ & none & mild & moderate & severe & $\begin{array}{l}\text { life-threatening or } \\
\text { disabling }\end{array}$ \\
\hline
\end{tabular}




\begin{tabular}{|c|c|c|c|c|c|}
\hline \multicolumn{6}{|c|}{ Grade } \\
\hline Adverse Event & $\mathbf{0}$ & 1 & 2 & 3 & 4 \\
\hline \multicolumn{6}{|c|}{ HEMORRHAGE } \\
\hline \multicolumn{6}{|c|}{ Notes: Transfusion in this section refers to $\mathrm{pRBC}$ infusion. } \\
\hline \multicolumn{6}{|c|}{$\begin{array}{l}\text { For any bleeding with grade } 3 \text { or } 4 \text { platelets }(<50,000) \text {, always grade Hemorrhage/bleeding with grade } 3 \text { or } 4 \text { thrombocytopenia. Also consider Platelets, } \\
\text { Transfusion: pRBCs, and Transfusion: platelets in addition to grading severity by grading the site or type of bleeding. }\end{array}$} \\
\hline \multicolumn{6}{|c|}{$\begin{array}{l}\text { If the site or type of Hemorrhage/bleeding is listed, also use the grading that incorporates the site of bleeding: CNS Hemorrhage/bleeding, Hematuria, } \\
\text { Hematemesis, Hemoptysis, Hemorrhage/bleeding with surgery, Melena/lower GI bleeding, Petechiae/purpura (Hemorrhage/bleeding into skin), Rectal } \\
\text { bleeding/hematochezia, Vaginal bleeding. }\end{array}$} \\
\hline \multicolumn{6}{|c|}{$\begin{array}{l}\text { If the platelet count is } \geq 50,000 \text { and the site or type of bleeding is listed, grade the specific site. If the site or type is not listed and the platelet count is } \\
\geq 50,000 \text {, grade Hemorrhage/bleeding without grade } 3 \text { or } 4 \text { thrombocytopenia and specify the site or type in the OTHER category. }\end{array}$} \\
\hline $\begin{array}{l}\text { Hemorrhage/bleeding with } \\
\text { grade } 3 \text { or } 4 \\
\text { thrombocytopenia }\end{array}$ & none & $\begin{array}{l}\text { mild without } \\
\text { transfusion }\end{array}$ & & requiring transfusion & $\begin{array}{l}\text { catastrophic bleeding, } \\
\text { requiring major non- } \\
\text { elective intervention }\end{array}$ \\
\hline \multicolumn{6}{|c|}{$\begin{array}{l}\text { Also consider Platelets, Hemoglobin, Transfusion: platelets, Transfusion: pRBCs, site or type of bleeding. If the site is not listed, grade as Hemorrhage-Other } \\
\text { (Specify site, __ }\end{array}$} \\
\hline \multicolumn{6}{|c|}{ ust be graded for any bleeding with grade $3 \mathrm{or}$} \\
\hline $\begin{array}{l}\text { Hemorrhage/bleeding } \\
\text { without grade } 3 \text { or } 4 \\
\text { thrombocytopenia }\end{array}$ & none & $\begin{array}{l}\text { mild without } \\
\text { transfusion }\end{array}$ & & requiring transfusion & $\begin{array}{l}\text { catastrophic bleeding } \\
\text { requiring major non- } \\
\text { elective intervention }\end{array}$ \\
\hline \multicolumn{6}{|c|}{ Also consider Platelets, Hemoglobin, Transfusion: platelets, Transfusion: pRBCs, Hemorrhage - Other (Specify site, _____ } \\
\hline \multicolumn{6}{|c|}{$\begin{array}{l}\text { Note: Bleeding in the absence of grade } 3 \text { or } 4 \text { thrombocytopenia is graded here only if the specific site or type of bleeding is not listed elsewhere in the } \\
\text { HEMORRHAGE category. Also grade as Other in the HEMORRHAGE category. }\end{array}$} \\
\hline CNS hemorrhage/bleeding & none & - & - & $\begin{array}{l}\text { bleeding noted on CT or } \\
\text { other scan with no } \\
\text { clinical consequences }\end{array}$ & $\begin{array}{l}\text { hemorrhagic stroke or } \\
\text { hemorrhagic vascular } \\
\text { event (CVA) with } \\
\text { neurologic signs and } \\
\text { symptoms }\end{array}$ \\
\hline Epistaxis & none & $\begin{array}{l}\text { mild without } \\
\text { transfusion }\end{array}$ & - & requiring transfusion & $\begin{array}{l}\text { catastrophic bleeding, } \\
\text { requiring major non- } \\
\text { elective intervention }\end{array}$ \\
\hline Hematemesis & none & $\begin{array}{l}\text { mild without } \\
\text { transfusion }\end{array}$ & - & requiring transfusion & $\begin{array}{l}\text { catastrophic bleeding, } \\
\text { requiring major non- } \\
\text { elective intervention }\end{array}$ \\
\hline $\begin{array}{l}\text { Hematuria } \\
\text { (in the absence of vaginal } \\
\text { bleeding) }\end{array}$ & none & microscopic only & $\begin{array}{l}\text { intermittent gross } \\
\text { bleeding, no clots }\end{array}$ & $\begin{array}{l}\text { persistent gross } \\
\text { bleeding or clots; may } \\
\text { require catheterization } \\
\text { or instrumentation, or } \\
\text { transfusion }\end{array}$ & $\begin{array}{l}\text { open surgery or necrosis } \\
\text { or deep bladder } \\
\text { ulceration }\end{array}$ \\
\hline Hemoptysis & none & $\begin{array}{l}\text { mild without } \\
\text { transfusion }\end{array}$ & - & requiring transfusion & $\begin{array}{l}\text { catastrophic bleeding, } \\
\text { requiring major non- } \\
\text { elective intervention }\end{array}$ \\
\hline $\begin{array}{l}\text { Hemorrhage/bleeding } \\
\text { associated with surgery }\end{array}$ & & $\begin{array}{l}\text { mild without } \\
\text { transfusion }\end{array}$ & - & requiring transfusion & $\begin{array}{l}\text { catastrophic bleeding, } \\
\text { requiring major non- } \\
\text { elective intervention }\end{array}$ \\
\hline \multicolumn{6}{|c|}{ Note: Expected blood loss at the time of surgery is not graded as an adverse event. } \\
\hline Melena/GI bleeding & none & $\begin{array}{l}\text { mild without } \\
\text { transfusion }\end{array}$ & - & requiring transfusion & $\begin{array}{l}\text { catastrophic bleeding, } \\
\text { requiring major non- } \\
\text { elective intervention }\end{array}$ \\
\hline
\end{tabular}


CTC Version 2.0

Publish Date: April 30, 1999

\begin{tabular}{|c|c|c|c|c|c|}
\hline \multicolumn{6}{|c|}{ Grade } \\
\hline Adverse Event & $\mathbf{0}$ & 1 & 2 & 3 & 4 \\
\hline $\begin{array}{l}\text { Petechiae/purpura } \\
\text { (hemorrhage/bleeding into } \\
\text { skin or mucosa) }\end{array}$ & none & rare petechiae of skin & $\begin{array}{l}\text { petechiae or purpura in } \\
\text { dependent areas of skin }\end{array}$ & $\begin{array}{l}\text { generalized petechiae or } \\
\text { purpura of skin or } \\
\text { petechiae of any } \\
\text { mucosal site }\end{array}$ & - \\
\hline $\begin{array}{l}\text { Rectal bleeding/ } \\
\text { hematochezia }\end{array}$ & none & $\begin{array}{l}\text { mild without } \\
\text { transfusion or } \\
\text { medication }\end{array}$ & $\begin{array}{l}\text { persistent, requiring } \\
\text { medication (e.g., steroid } \\
\text { suppositories) and/or } \\
\text { break from radiation } \\
\text { treatment }\end{array}$ & requiring transfusion & $\begin{array}{l}\text { catastrophic bleeding, } \\
\text { requiring major non- } \\
\text { elective intervention }\end{array}$ \\
\hline Vaginal bleeding & none & $\begin{array}{l}\text { spotting, requiring }<2 \\
\text { pads per day }\end{array}$ & $\begin{array}{l}\text { requiring } \geq 2 \text { pads per } \\
\text { day, but not requiring } \\
\text { transfusion }\end{array}$ & requiring transfusion & $\begin{array}{l}\text { catastrophic bleeding, } \\
\text { requiring major non- } \\
\text { elective intervention }\end{array}$ \\
\hline $\begin{array}{l}\text { Hemorrhage - Other } \\
\text { (Specify site, }\end{array}$ & none & $\begin{array}{l}\text { mild without } \\
\text { transfusion }\end{array}$ & - & requiring transfusion & $\begin{array}{l}\text { catastrophic bleeding, } \\
\text { requiring major non- } \\
\text { elective intervention }\end{array}$ \\
\hline \multicolumn{6}{|c|}{ HEPATIC } \\
\hline Alkaline phosphatase & WNL & $>\mathrm{ULN}-2.5 \mathrm{x}$ ULN & $>2.5-5.0 \times \mathrm{ULN}$ & $>5.0-20.0 \times$ ULN & $>20.0 \times$ ULN \\
\hline Bilirubin & WNL & $>\mathrm{ULN}-1.5 \mathrm{x}$ ULN & $>1.5-3.0 \times$ ULN & $>3.0-10.0 \times \mathrm{ULN}$ & $>10.0 \times$ ULN \\
\hline $\begin{array}{l}\text { Bilirubin associated with } \\
\text { graft versus host disease } \\
\text { (GVHD) for BMT studies, if } \\
\text { specified in the protocol. }\end{array}$ & normal & $\geq 2-<3 \mathrm{mg} / 100 \mathrm{~mL}$ & $\geq 3-<6 \mathrm{mg} / 100 \mathrm{~mL}$ & $\geq 6-<15 \mathrm{mg} / 100 \mathrm{~mL}$ & $\geq 15 \mathrm{mg} / 100 \mathrm{~mL}$ \\
\hline $\begin{array}{l}\text { GGT } \\
(\gamma \text { - Glutamyl transpeptidase })\end{array}$ & WNL & $>\mathrm{ULN}-2.5 \times \mathrm{ULN}$ & $>2.5-5.0 \times$ ULN & $>5.0-20.0 \times \mathrm{ULN}$ & $>20.0 \times$ ULN \\
\hline Hepatic enlargement & absent & - & - & present & - \\
\hline Hypoalbuminemia & WNL & $<\mathrm{LLN}-3 \mathrm{~g} / \mathrm{dL}$ & $\geq 2-<3 \mathrm{~g} / \mathrm{dL}$ & $<2 \mathrm{~g} / \mathrm{dL}$ & - \\
\hline $\begin{array}{l}\text { Liver dysfunction/ failure } \\
\text { (clinical) }\end{array}$ & normal & - & - & asterixis & encephalopathy or coma \\
\hline Portal vein flow & normal & - & $\begin{array}{l}\text { decreased portal vein } \\
\text { flow }\end{array}$ & $\begin{array}{l}\text { reversal/retrograde } \\
\text { portal vein flow }\end{array}$ & - \\
\hline $\begin{array}{l}\text { SGOT (AST) } \\
\text { (serum glutamic oxaloacetic } \\
\text { transaminase) }\end{array}$ & WNL & $>\mathrm{ULN}-2.5 \times \mathrm{ULN}$ & $>2.5-5.0 \times \mathrm{ULN}$ & $>5.0-20.0 \times \mathrm{ULN}$ & $>20.0 \times$ ULN \\
\hline $\begin{array}{l}\text { SGPT (ALT) } \\
\text { (serum glutamic pyruvic } \\
\text { transaminase) }\end{array}$ & WNL & $>\mathrm{ULN}-2.5 \mathrm{x}$ ULN & $>2.5-5.0 \times \mathrm{ULN}$ & $>5.0-20.0 \times$ ULN & $>20.0 \times$ ULN \\
\hline $\begin{array}{l}\text { Hepatic - Other } \\
\text { (Specify, }\end{array}$ & none & mild & moderate & severe & $\begin{array}{l}\text { life-threatening or } \\
\text { disabling }\end{array}$ \\
\hline \multicolumn{6}{|c|}{ INFECTION/FEBRILE NEUTROPENIA } \\
\hline Catheter-related infection & none & $\begin{array}{l}\text { mild, no active } \\
\text { treatment }\end{array}$ & $\begin{array}{l}\text { moderate, localized } \\
\text { infection, requiring } \\
\text { local or oral treatment }\end{array}$ & $\begin{array}{l}\text { severe, systemic } \\
\text { infection, requiring IV } \\
\text { antibiotic or antifungal } \\
\text { treatment or } \\
\text { hospitalization }\end{array}$ & $\begin{array}{l}\text { life-threatening sepsis } \\
\text { (e.g., septic shock) }\end{array}$ \\
\hline
\end{tabular}


CTC Version 2.0

Publish Date: April 30, 1999

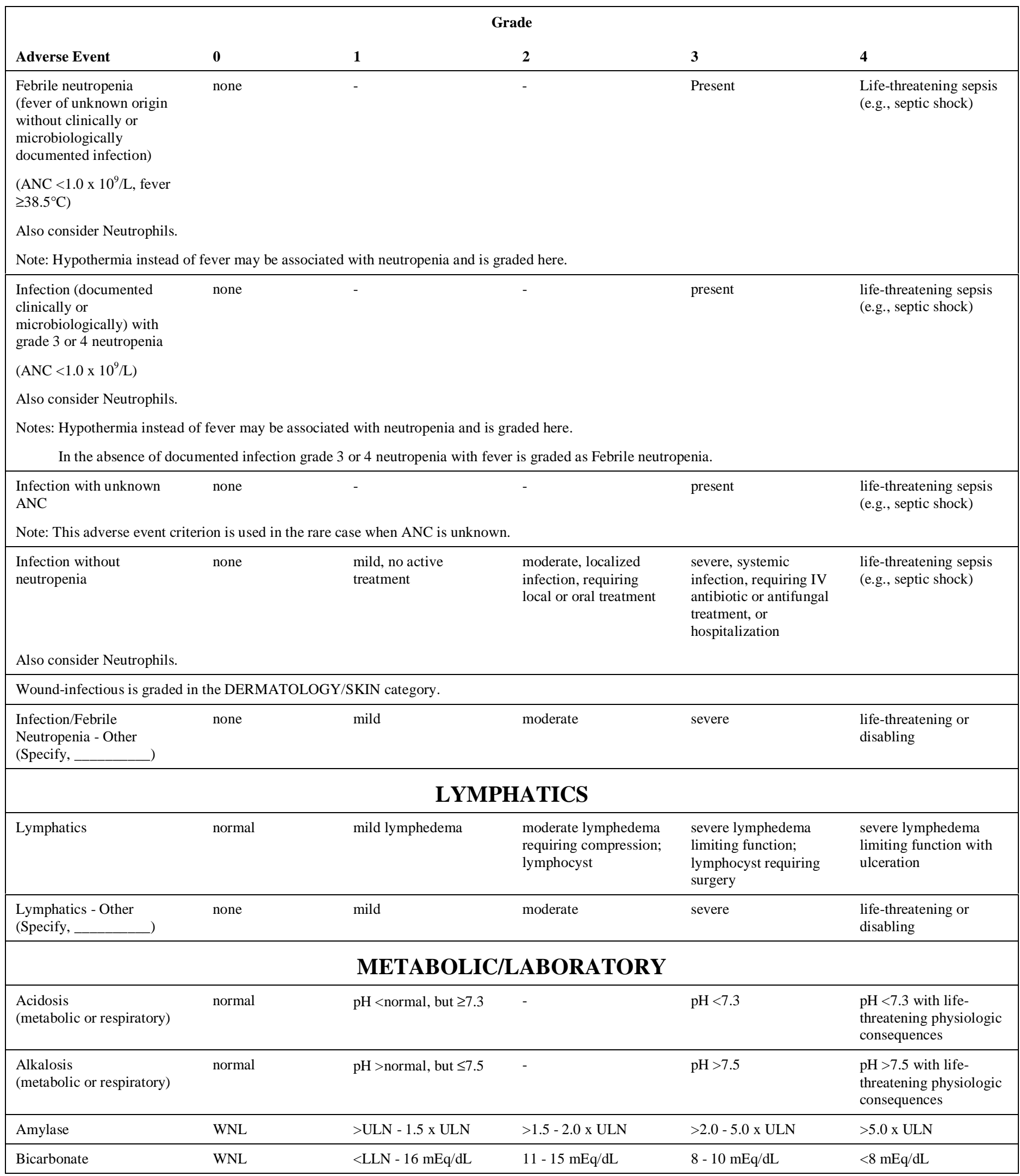


CTC Version 2.0

Publish Date: April 30, 1999

\begin{tabular}{|c|c|c|c|c|c|}
\hline \multicolumn{6}{|c|}{ Grade } \\
\hline Adverse Event & $\mathbf{0}$ & 1 & 2 & 3 & 4 \\
\hline $\begin{array}{l}\text { CPK } \\
\text { (creatine phosphokinase) }\end{array}$ & WNL & $>\mathrm{ULN}-2.5 \times \mathrm{ULN}$ & $>2.5-5 \times$ ULN & $>5-10 \times \mathrm{ULN}$ & $>10 \times$ ULN \\
\hline Hypercalcemia & WNL & $\begin{array}{l}>\mathrm{ULN}-11.5 \mathrm{mg} / \mathrm{dL} \\
>\mathrm{ULN}-2.9 \mathrm{mmol} / \mathrm{L}\end{array}$ & $\begin{array}{l}>11.5-12.5 \mathrm{mg} / \mathrm{dL} \\
>2.9-3.1 \mathrm{mmol} / \mathrm{L}\end{array}$ & $\begin{array}{l}>12.5-13.5 \mathrm{mg} / \mathrm{dL} \\
>3.1-3.4 \mathrm{mmol} / \mathrm{L}\end{array}$ & $\begin{array}{l}>13.5 \mathrm{mg} / \mathrm{dL} \\
>3.4 \mathrm{mmol} / \mathrm{L}\end{array}$ \\
\hline Hypercholesterolemia & WNL & $\begin{array}{l}>\mathrm{ULN}-300 \mathrm{mg} / \mathrm{dL} \\
>\mathrm{ULN}-7.75 \mathrm{mmol} / \mathrm{L}\end{array}$ & $\begin{array}{l}>300-400 \mathrm{mg} / \mathrm{dL} \\
>7.75-10.34 \mathrm{mmol} / \mathrm{L}\end{array}$ & $\begin{array}{l}>400-500 \mathrm{mg} / \mathrm{dL} \\
>10.34-12.92 \mathrm{mmol} / \mathrm{L}\end{array}$ & $\begin{array}{l}>500 \mathrm{mg} / \mathrm{dL} \\
>12.92 \mathrm{mmol} / \mathrm{L}\end{array}$ \\
\hline Hyperglycemia & WNL & $\begin{array}{l}>\mathrm{ULN}-160 \mathrm{mg} / \mathrm{dL} \\
>\mathrm{ULN}-8.9 \mathrm{mmol} / \mathrm{L}\end{array}$ & $\begin{array}{l}>160-250 \mathrm{mg} / \mathrm{dL} \\
>8.9-13.9 \mathrm{mmol} / \mathrm{L}\end{array}$ & $\begin{array}{l}>250-500 \mathrm{mg} / \mathrm{dL} \\
>13.9-27.8 \mathrm{mmol} / \mathrm{L}\end{array}$ & $\begin{array}{l}>500 \mathrm{mg} / \mathrm{dL} \\
>27.8 \mathrm{mmol} / \mathrm{L} \text { or } \\
\text { acidosis }\end{array}$ \\
\hline Hyperkalemia & WNL & $>\mathrm{ULN}-5.5 \mathrm{mmol} / \mathrm{L}$ & $>5.5-6.0 \mathrm{mmol} / \mathrm{L}$ & $>6.0-7.0 \mathrm{mmol} / \mathrm{L}$ & $>7.0 \mathrm{mmol} / \mathrm{L}$ \\
\hline Hypermagnesemia & WNL & $\begin{array}{l}>\mathrm{ULN}-3.0 \mathrm{mg} / \mathrm{dL} \\
>\mathrm{ULN}-1.23 \mathrm{mmol} / \mathrm{L}\end{array}$ & - & $\begin{array}{l}>3.0-8.0 \mathrm{mg} / \mathrm{dL} \\
>1.23-3.30 \mathrm{mmol} / \mathrm{L}\end{array}$ & $\begin{array}{l}>8.0 \mathrm{mg} / \mathrm{dL} \\
>3.30 \mathrm{mmol} / \mathrm{L}\end{array}$ \\
\hline Hypernatremia & WNL & $>\mathrm{ULN}-150 \mathrm{mmol} / \mathrm{L}$ & $>150-155 \mathrm{mmol} / \mathrm{L}$ & $>155-160 \mathrm{mmol} / \mathrm{L}$ & $>160 \mathrm{mmol} / \mathrm{L}$ \\
\hline Hypertriglyceridemia & WNL & $>\mathrm{ULN}-2.5 \times \mathrm{ULN}$ & $>2.5-5.0 \times \mathrm{ULN}$ & $>5.0-10 \times$ ULN & $>10 \times$ ULN \\
\hline Hyperuricemia & WNL & $\begin{array}{l}>\mathrm{ULN}-\leq 10 \mathrm{mg} / \mathrm{dL} \\
\leq 0.59 \mathrm{mmol} / \mathrm{L} \text { without } \\
\text { physiologic } \\
\text { consequences }\end{array}$ & - & $\begin{array}{l}>\mathrm{ULN}-\leq 10 \mathrm{mg} / \mathrm{dL} \\
\leq 0.59 \mathrm{mmol} / \mathrm{L} \text { with } \\
\text { physiologic } \\
\text { consequences }\end{array}$ & $\begin{array}{l}>10 \mathrm{mg} / \mathrm{dL} \\
>0.59 \mathrm{mmol} / \mathrm{L}\end{array}$ \\
\hline Hypocalcemia & WNL & $\begin{array}{l}<\mathrm{LLN}-8.0 \mathrm{mg} / \mathrm{dL} \\
<\mathrm{LLN}-2.0 \mathrm{mmol} / \mathrm{L}\end{array}$ & $\begin{array}{l}7.0-<8.0 \mathrm{mg} / \mathrm{dL} \\
1.75-<2.0 \mathrm{mmol} / \mathrm{L}\end{array}$ & $\begin{array}{l}6.0-<7.0 \mathrm{mg} / \mathrm{dL} \\
1.5-<1.75 \mathrm{mmol} / \mathrm{L}\end{array}$ & $\begin{array}{l}<6.0 \mathrm{mg} / \mathrm{dL} \\
<1.5 \mathrm{mmol} / \mathrm{L}\end{array}$ \\
\hline Hypoglycemia & WNL & $\begin{array}{l}<\mathrm{LLN}-55 \mathrm{mg} / \mathrm{dL} \\
<\mathrm{LLN}-3.0 \mathrm{mmol} / \mathrm{L}\end{array}$ & $\begin{array}{l}40-<55 \mathrm{mg} / \mathrm{dL} \\
2.2-<3.0 \mathrm{mmol} / \mathrm{L}\end{array}$ & $\begin{array}{l}30-<40 \mathrm{mg} / \mathrm{dL} \\
1.7-<2.2 \mathrm{mmol} / \mathrm{L}\end{array}$ & $\begin{array}{l}<30 \mathrm{mg} / \mathrm{dL} \\
<1.7 \mathrm{mmol} / \mathrm{L}\end{array}$ \\
\hline Hypokalemia & WNL & $<\mathrm{LLN}-3.0 \mathrm{mmol} / \mathrm{L}$ & - & $2.5-<3.0 \mathrm{mmol} / \mathrm{L}$ & $<2.5 \mathrm{mmol} / \mathrm{L}$ \\
\hline Hypomagnesemia & WNL & $\begin{array}{l}<\mathrm{LLN}-1.2 \mathrm{mg} / \mathrm{dL} \\
<\mathrm{LLN}-0.5 \mathrm{mmol} / \mathrm{L}\end{array}$ & $\begin{array}{l}0.9-<1.2 \mathrm{mg} / \mathrm{dL} \\
0.4-<0.5 \mathrm{mmol} / \mathrm{L}\end{array}$ & $\begin{array}{l}0.7-<0.9 \mathrm{mg} / \mathrm{dL} \\
0.3-<0.4 \mathrm{mmol} / \mathrm{L}\end{array}$ & $\begin{array}{l}<0.7 \mathrm{mg} / \mathrm{dL} \\
<0.3 \mathrm{mmol} / \mathrm{L}\end{array}$ \\
\hline Hyponatremia & WNL & $<\mathrm{LLN}-130 \mathrm{mmol} / \mathrm{L}$ & - & $120-<130 \mathrm{mmol} / \mathrm{L}$ & $<120 \mathrm{mmol} / \mathrm{L}$ \\
\hline Hypophosphatemia & WNL & $\begin{array}{l}<\mathrm{LLN}-2.5 \mathrm{mg} / \mathrm{dL} \\
<\mathrm{LLN}-0.8 \mathrm{mmol} / \mathrm{L}\end{array}$ & $\begin{array}{l}\geq 2.0-<2.5 \mathrm{mg} / \mathrm{dL} \\
\geq 0.6-<0.8 \mathrm{mmol} / \mathrm{L}\end{array}$ & $\begin{array}{l}\geq 1.0-<2.0 \mathrm{mg} / \mathrm{dL} \\
\geq 0.3-<0.6 \mathrm{mmol} / \mathrm{L}\end{array}$ & $\begin{array}{l}<1.0 \mathrm{mg} / \mathrm{dL} \\
<0.3 \mathrm{mmol} / \mathrm{L}\end{array}$ \\
\hline \multicolumn{6}{|c|}{ Hypothyroidism is graded in the ENDOCRINE category. } \\
\hline Lipase & WNL & $>\mathrm{ULN}-1.5 \times \mathrm{ULN}$ & $>1.5-2.0 \times \mathrm{ULN}$ & $>2.0-5.0 \times \mathrm{x}$ ULN & $>5.0 \times$ ULN \\
\hline $\begin{array}{l}\text { Metabolic/Laboratory - } \\
\text { Other (Specify, }\end{array}$ & none & mild & moderate & severe & $\begin{array}{l}\text { life-threatening or } \\
\text { disabling }\end{array}$ \\
\hline \multicolumn{6}{|c|}{ MUSCULOSKELETAL } \\
\hline \multicolumn{6}{|c|}{ Arthralgia is graded in the PAIN category. } \\
\hline Arthritis & none & $\begin{array}{l}\text { mild pain with } \\
\text { inflammation, erythema } \\
\text { or joint swelling but not } \\
\text { interfering with } \\
\text { function }\end{array}$ & $\begin{array}{l}\text { moderate pain with } \\
\text { inflammation, } \\
\text { erythema, or joint } \\
\text { swelling interfering } \\
\text { with function, but not } \\
\text { interfering with } \\
\text { activities of daily living }\end{array}$ & $\begin{array}{l}\text { severe pain with } \\
\text { inflammation, } \\
\text { erythema, or joint } \\
\text { swelling and interfering } \\
\text { with activities of daily } \\
\text { living }\end{array}$ & disabling \\
\hline
\end{tabular}


CTC Version 2.0

Publish Date: April 30, 1999

\begin{tabular}{|c|c|c|c|c|c|}
\hline \multicolumn{6}{|c|}{ Grade } \\
\hline Adverse Event & $\mathbf{0}$ & 1 & 2 & 3 & 4 \\
\hline $\begin{array}{l}\text { Muscle weakness } \\
\text { (not due to neuropathy) }\end{array}$ & normal & $\begin{array}{l}\text { asymptomatic with } \\
\text { weakness on physical } \\
\text { exam }\end{array}$ & $\begin{array}{l}\text { symptomatic and } \\
\text { interfering with } \\
\text { function, but not } \\
\text { interfering with } \\
\text { activities of daily living }\end{array}$ & $\begin{array}{l}\text { symptomatic and } \\
\text { interfering with } \\
\text { activities of daily living }\end{array}$ & bedridden or disabling \\
\hline \multicolumn{6}{|c|}{ Myalgia [tenderness or pain in muscles] is graded in the PAIN category. } \\
\hline $\begin{array}{l}\text { Myositis } \\
\text { (inflammation/damage of } \\
\text { muscle) }\end{array}$ & none & $\begin{array}{l}\text { mild pain, not } \\
\text { interfering with } \\
\text { function }\end{array}$ & $\begin{array}{l}\text { pain interfering with } \\
\text { function, but not } \\
\text { interfering with } \\
\text { activities of daily living }\end{array}$ & $\begin{array}{l}\text { pain interfering with } \\
\text { function and interfering } \\
\text { with activities of daily } \\
\text { living }\end{array}$ & bedridden or disabling \\
\hline \multicolumn{6}{|l|}{ Also consider CPK. } \\
\hline \multicolumn{6}{|c|}{ Note: Myositis implies muscle damage (i.e., elevated CPK). } \\
\hline $\begin{array}{l}\text { Osteonecrosis } \\
\text { (avascular necrosis) }\end{array}$ & none & $\begin{array}{l}\text { asymptomatic and } \\
\text { detected by imaging } \\
\text { only }\end{array}$ & $\begin{array}{l}\text { symptomatic and } \\
\text { interfering with } \\
\text { function, but not } \\
\text { interfering with } \\
\text { activities of daily living }\end{array}$ & $\begin{array}{l}\text { symptomatic and } \\
\text { interfering with } \\
\text { activities of daily living }\end{array}$ & $\begin{array}{l}\text { symptomatic; or } \\
\text { disabling }\end{array}$ \\
\hline $\begin{array}{l}\text { Musculoskeletal - Other } \\
\text { (Specify, }\end{array}$ & none & mild & moderate & severe & $\begin{array}{l}\text { life-threatening or } \\
\text { disabling }\end{array}$ \\
\hline \multicolumn{6}{|c|}{ NEUROLOGY } \\
\hline \multicolumn{6}{|c|}{ Aphasia, receptive and/or expressive, is graded under Speech impairment in the NEUROLOGY category. } \\
\hline $\begin{array}{l}\text { Arachnoiditis/meningismus/ } \\
\text { radiculitis }\end{array}$ & absent & $\begin{array}{l}\text { mild pain not interfering } \\
\text { with function }\end{array}$ & $\begin{array}{l}\text { moderate pain } \\
\text { interfering with } \\
\text { function, but not } \\
\text { interfering with } \\
\text { activities of daily living }\end{array}$ & $\begin{array}{l}\text { severe pain interfering } \\
\text { with activities of daily } \\
\text { living }\end{array}$ & $\begin{array}{l}\text { unable to function or } \\
\text { perform activities of } \\
\text { daily living; bedridden; } \\
\text { paraplegia }\end{array}$ \\
\hline \multicolumn{6}{|c|}{ Also consider Headache, Vomiting, Fever. } \\
\hline Ataxia (incoordination) & normal & $\begin{array}{l}\text { asymptomatic but } \\
\text { abnormal on physical } \\
\text { exam, and not } \\
\text { interfering with } \\
\text { function }\end{array}$ & $\begin{array}{l}\text { mild symptoms } \\
\text { interfering with } \\
\text { function, but not } \\
\text { interfering with } \\
\text { activities of daily living }\end{array}$ & $\begin{array}{l}\text { moderate symptoms } \\
\text { interfering with } \\
\text { activities of daily living }\end{array}$ & bedridden or disabling \\
\hline $\begin{array}{l}\text { CNS cerebrovascular } \\
\text { ischemia }\end{array}$ & none & - & - & $\begin{array}{l}\text { transient ischemic event } \\
\text { or attack (TIA) }\end{array}$ & $\begin{array}{l}\text { permanent event (e.g., } \\
\text { cerebral vascular } \\
\text { accident) }\end{array}$ \\
\hline \multicolumn{6}{|c|}{ CNS hemorrhage/bleeding is graded in the HEMORRHAGE category. } \\
\hline $\begin{array}{l}\text { Cognitive disturbancel } \\
\text { learning problems }\end{array}$ & none & $\begin{array}{l}\text { cognitive disability; not } \\
\text { interfering with } \\
\text { work/school } \\
\text { performance; } \\
\text { preservation of } \\
\text { intelligence }\end{array}$ & $\begin{array}{l}\text { cognitive disability; } \\
\text { interfering with } \\
\text { work/school } \\
\text { performance; decline of } \\
1 \text { SD (Standard } \\
\text { Deviation) or loss of } \\
\text { developmental } \\
\text { milestones }\end{array}$ & $\begin{array}{l}\text { cognitive disability; } \\
\text { resulting in significant } \\
\text { impairment of } \\
\text { work/school } \\
\text { performance; cognitive } \\
\text { decline }>2 S D\end{array}$ & $\begin{array}{l}\text { inability to work/frank } \\
\text { mental retardation }\end{array}$ \\
\hline
\end{tabular}


CTC Version 2.0

Publish Date: April 30, 1999

\begin{tabular}{|c|c|c|c|c|c|}
\hline \multicolumn{6}{|c|}{ Grade } \\
\hline Adverse Event & $\mathbf{0}$ & 1 & 2 & 3 & 4 \\
\hline Confusion & normal & $\begin{array}{l}\text { confusion or } \\
\text { disorientation or } \\
\text { attention deficit of brief } \\
\text { duration; resolves } \\
\text { spontaneously with no } \\
\text { sequelae }\end{array}$ & $\begin{array}{l}\text { confusion or } \\
\text { disorientation or } \\
\text { attention deficit } \\
\text { interfering with } \\
\text { function, but not } \\
\text { interfering with } \\
\text { activities of daily living }\end{array}$ & $\begin{array}{l}\text { confusion or delirium } \\
\text { interfering with } \\
\text { activities of daily living }\end{array}$ & $\begin{array}{l}\text { harmful to others or } \\
\text { self; requiring } \\
\text { hospitalization }\end{array}$ \\
\hline \multicolumn{6}{|c|}{ Cranial neuropathy is graded in the NEUROLOGY category as Neuropathy-cranial. } \\
\hline Delusions & normal & - & - & present & toxic psychosis \\
\hline $\begin{array}{l}\text { Depressed level of } \\
\text { consciousness }\end{array}$ & normal & $\begin{array}{l}\text { somnolence or sedation } \\
\text { not interfering with } \\
\text { function }\end{array}$ & $\begin{array}{l}\text { somnolence or sedation } \\
\text { interfering with } \\
\text { function, but not } \\
\text { interfering with } \\
\text { activities of daily living }\end{array}$ & $\begin{array}{l}\text { obtundation or stupor; } \\
\text { difficult to arouse; } \\
\text { interfering with } \\
\text { activities of daily living }\end{array}$ & coma \\
\hline \multicolumn{6}{|c|}{ Note: Syncope (fainting) is graded in the NEUROLOGY category. } \\
\hline Dizziness/lightheadedness & none & $\begin{array}{l}\text { not interfering with } \\
\text { function }\end{array}$ & $\begin{array}{l}\text { interfering with } \\
\text { function, but not } \\
\text { interfering with } \\
\text { activities of daily living }\end{array}$ & $\begin{array}{l}\text { interfering with } \\
\text { activities of daily living }\end{array}$ & bedridden or disabling \\
\hline \multicolumn{6}{|c|}{ Dysphasia, receptive and/or expressive, is graded under Speech impairment in the NEUROLOGY category. } \\
\hline $\begin{array}{l}\text { Extrapyramidal/ } \\
\text { involuntary movement/ } \\
\text { restlessness }\end{array}$ & none & $\begin{array}{l}\text { mild involuntary } \\
\text { movements not } \\
\text { interfering with } \\
\text { function }\end{array}$ & $\begin{array}{l}\text { moderate involuntary } \\
\text { movements interfering } \\
\text { with function, but not } \\
\text { interfering with } \\
\text { activities of daily living }\end{array}$ & $\begin{array}{l}\text { severe involuntary } \\
\text { movements or torticollis } \\
\text { interfering with } \\
\text { activities of daily living }\end{array}$ & bedridden or disabling \\
\hline Hallucinations & normal & - & - & present & toxic psychosis \\
\hline \multicolumn{6}{|c|}{ Headache is graded in the PAIN category. } \\
\hline Insomnia & normal & $\begin{array}{l}\text { occasional difficulty } \\
\text { sleeping not interfering } \\
\text { with function }\end{array}$ & $\begin{array}{l}\text { difficulty sleeping } \\
\text { interfering with } \\
\text { function, but not } \\
\text { interfering with } \\
\text { activities of daily living }\end{array}$ & $\begin{array}{l}\text { frequent difficulty } \\
\text { sleeping, interfering } \\
\text { with activities of daily } \\
\text { living }\end{array}$ & - \\
\hline $\begin{array}{l}\text { Irritability } \\
\text { (children }<3 \text { years of age) }\end{array}$ & normal & mild; easily consolable & $\begin{array}{l}\text { moderate; requiring } \\
\text { increased attention }\end{array}$ & severe; inconsolable & - \\
\hline $\begin{array}{l}\text { Leukoencephalopathy } \\
\text { associated radiological } \\
\text { findings }\end{array}$ & none & $\begin{array}{l}\text { mild increase in SAS } \\
\text { (subarachnoid space) } \\
\text { and/or mild } \\
\text { ventriculomegaly; } \\
\text { and/or small (+/- } \\
\text { multiple) focal T2 } \\
\text { hyperintensities, } \\
\text { involving } \\
\text { periventricular white } \\
\text { matter or <1/3 of } \\
\text { susceptible areas of } \\
\text { cerebrum }\end{array}$ & $\begin{array}{l}\text { moderate increase in } \\
\text { SAS; and/or moderate } \\
\text { ventriculomegaly; } \\
\text { and/or focal T2 } \\
\text { hyperintensities } \\
\text { extending into centrum } \\
\text { ovale; or involving } 1 / 3 \\
\text { to } 2 / 3 \text { of susceptible } \\
\text { areas of cerebrum }\end{array}$ & $\begin{array}{l}\text { severe increase in SAS; } \\
\text { severe } \\
\text { ventriculomegaly; near } \\
\text { total white matter T2 } \\
\text { hyperintensities or } \\
\text { diffuse low attenuation } \\
\text { (CT); focal white matter } \\
\text { necrosis (cystic) }\end{array}$ & $\begin{array}{l}\text { severe increase in SAS; } \\
\text { severe } \\
\text { ventriculomegaly; } \\
\text { diffuse low attenuation } \\
\text { with calcification }(\mathrm{CT}) \text {; } \\
\text { diffuse white matter } \\
\text { necrosis (MRI) }\end{array}$ \\
\hline Memory loss & normal & $\begin{array}{l}\text { memory loss not } \\
\text { interfering with } \\
\text { function }\end{array}$ & $\begin{array}{l}\text { memory loss interfering } \\
\text { with function, but not } \\
\text { interfering with } \\
\text { activities of daily living }\end{array}$ & $\begin{array}{l}\text { memory loss interfering } \\
\text { with activities of daily } \\
\text { living }\end{array}$ & amnesia \\
\hline
\end{tabular}


CTC Version 2.0

Publish Date: April 30, 1999

\begin{tabular}{|c|c|c|c|c|c|}
\hline \multicolumn{6}{|c|}{ Grade } \\
\hline Adverse Event & $\mathbf{0}$ & 1 & 2 & 3 & 4 \\
\hline $\begin{array}{l}\text { Mood alteration-anxiety, } \\
\text { agitation }\end{array}$ & normal & $\begin{array}{l}\text { mild mood alteration } \\
\text { not interfering with } \\
\text { function }\end{array}$ & $\begin{array}{l}\text { moderate mood } \\
\text { alteration interfering } \\
\text { with function, but not } \\
\text { interfering with } \\
\text { activities of daily living }\end{array}$ & $\begin{array}{l}\text { severe mood alteration } \\
\text { interfering with } \\
\text { activities of daily living }\end{array}$ & $\begin{array}{l}\text { suicidal ideation or } \\
\text { danger to self }\end{array}$ \\
\hline Mood alteration-depression & normal & $\begin{array}{l}\text { mild mood alteration } \\
\text { not interfering with } \\
\text { function }\end{array}$ & $\begin{array}{l}\text { moderate mood } \\
\text { alteration interfering } \\
\text { with function, but not } \\
\text { interfering with } \\
\text { activities of daily living }\end{array}$ & $\begin{array}{l}\text { severe mood alteration } \\
\text { interfering with } \\
\text { activities of daily living }\end{array}$ & $\begin{array}{l}\text { suicidal ideation or } \\
\text { danger to self }\end{array}$ \\
\hline Mood alteration-euphoria & normal & $\begin{array}{l}\text { mild mood alteration } \\
\text { not interfering with } \\
\text { function }\end{array}$ & $\begin{array}{l}\text { moderate mood } \\
\text { alteration interfering } \\
\text { with function, but not } \\
\text { interfering with } \\
\text { activities of daily living }\end{array}$ & $\begin{array}{l}\text { severe mood alteration } \\
\text { interfering with } \\
\text { activities of daily living }\end{array}$ & danger to self \\
\hline \multicolumn{6}{|c|}{ Neuropathic pain is graded in the PAIN category. } \\
\hline Neuropathy-cranial & absent & - & $\begin{array}{l}\text { present, not interfering } \\
\text { with activities of daily } \\
\text { living }\end{array}$ & $\begin{array}{l}\text { present, interfering with } \\
\text { activities of daily living }\end{array}$ & $\begin{array}{l}\text { life-threatening, } \\
\text { disabling }\end{array}$ \\
\hline Neuropathy-motor & normal & $\begin{array}{l}\text { subjective weakness but } \\
\text { no objective findings }\end{array}$ & $\begin{array}{l}\text { mild objective } \\
\text { weakness interfering } \\
\text { with function, but not } \\
\text { interfering with } \\
\text { activities of daily living }\end{array}$ & $\begin{array}{l}\text { objective weakness } \\
\text { interfering with } \\
\text { activities of daily living }\end{array}$ & paralysis \\
\hline Neuropathy-sensory & normal & $\begin{array}{l}\text { loss of deep tendon } \\
\text { reflexes or paresthesia } \\
\text { (including tingling) but } \\
\text { not interfering with } \\
\text { function }\end{array}$ & $\begin{array}{l}\text { objective sensory loss } \\
\text { or paresthesia } \\
\text { (including tingling), } \\
\text { interfering with } \\
\text { function, but not } \\
\text { interfering with } \\
\text { activities of daily living }\end{array}$ & $\begin{array}{l}\text { sensory loss or } \\
\text { paresthesia interfering } \\
\text { with activities of daily } \\
\text { living }\end{array}$ & $\begin{array}{l}\text { permanent sensory loss } \\
\text { that interferes with } \\
\text { function }\end{array}$ \\
\hline $\begin{array}{l}\text { Nystagmus } \\
\text { Also consider Vision-double }\end{array}$ & $\begin{array}{l}\text { absent } \\
\text { sion. }\end{array}$ & present & - & - & - \\
\hline Personality/behavioral & normal & $\begin{array}{l}\text { change, but not } \\
\text { disruptive to patient or } \\
\text { family }\end{array}$ & $\begin{array}{l}\text { disruptive to patient or } \\
\text { family }\end{array}$ & $\begin{array}{l}\text { disruptive to patient and } \\
\text { family; requiring mental } \\
\text { health intervention }\end{array}$ & $\begin{array}{l}\text { harmful to others or } \\
\text { self; requiring } \\
\text { hospitalization }\end{array}$ \\
\hline $\begin{array}{l}\text { Pyramidal tract dysfunction } \\
\text { (e.g., } \uparrow \text { tone, hyperreflexia, } \\
\text { positive Babinski, } \downarrow \text { fine } \\
\text { motor coordination) }\end{array}$ & normal & $\begin{array}{l}\text { asymptomatic with } \\
\text { abnormality on physical } \\
\text { examination }\end{array}$ & $\begin{array}{l}\text { symptomatic or } \\
\text { interfering with } \\
\text { function but not } \\
\text { interfering with } \\
\text { activities of daily living }\end{array}$ & $\begin{array}{l}\text { interfering with } \\
\text { activities of daily living }\end{array}$ & $\begin{array}{l}\text { bedridden or disabling; } \\
\text { paralysis }\end{array}$ \\
\hline Seizure(s) & none & - & $\begin{array}{l}\text { seizure(s) self-limited } \\
\text { and consciousness is } \\
\text { preserved }\end{array}$ & $\begin{array}{l}\text { seizure(s) in which } \\
\text { consciousness is altered }\end{array}$ & $\begin{array}{l}\text { seizures of any type } \\
\text { which are prolonged, } \\
\text { repetitive, or difficult to } \\
\text { control (e.g., status } \\
\text { epilepticus, intractable } \\
\text { epilepsy) }\end{array}$ \\
\hline $\begin{array}{l}\text { Speech impairment } \\
\text { (e.g., dysphasia or aphasia) }\end{array}$ & normal & - & $\begin{array}{l}\text { awareness of receptive } \\
\text { or expressive dysphasia, } \\
\text { not impairing ability to } \\
\text { communicate }\end{array}$ & $\begin{array}{l}\text { receptive or expressive } \\
\text { dysphasia, impairing } \\
\text { ability to communicate }\end{array}$ & $\begin{array}{l}\text { inability to } \\
\text { communicate }\end{array}$ \\
\hline Syncope (fainting) & absent & - & - & present & - \\
\hline
\end{tabular}


CTC Version 2.0

Publish Date: April 30, 1999

\begin{tabular}{|c|c|c|c|c|c|}
\hline \multicolumn{6}{|c|}{ Grade } \\
\hline Adverse Event & $\mathbf{0}$ & 1 & 2 & 3 & 4 \\
\hline Tremor & none & $\begin{array}{l}\text { mild and brief or } \\
\text { intermittent but not } \\
\text { interfering with } \\
\text { function }\end{array}$ & $\begin{array}{l}\text { moderate tremor } \\
\text { interfering with } \\
\text { function, but not } \\
\text { interfering with } \\
\text { activities of daily living }\end{array}$ & $\begin{array}{l}\text { severe tremor } \\
\text { interfering with } \\
\text { activities of daily living }\end{array}$ & - \\
\hline Vertigo & none & $\begin{array}{l}\text { not interfering with } \\
\text { function }\end{array}$ & $\begin{array}{l}\text { interfering with } \\
\text { function, but not } \\
\text { interfering with } \\
\text { activities of daily living }\end{array}$ & $\begin{array}{l}\text { interfering with } \\
\text { activities of daily living }\end{array}$ & bedridden or disabling \\
\hline $\begin{array}{l}\text { Neurology - Other } \\
\text { (Specify, }\end{array}$ & none & mild & moderate & severe & $\begin{array}{l}\text { life-threatening or } \\
\text { disabling }\end{array}$ \\
\hline \multicolumn{6}{|c|}{ OCULAR/VISUAL } \\
\hline Cataract & none & asymptomatic & $\begin{array}{l}\text { symptomatic, partial } \\
\text { visual loss }\end{array}$ & $\begin{array}{l}\text { symptomatic, visual } \\
\text { loss requiring treatment } \\
\text { or interfering with } \\
\text { function }\end{array}$ & - \\
\hline Conjunctivitis & none & $\begin{array}{l}\text { abnormal } \\
\text { ophthalmologic } \\
\text { changes, but } \\
\text { asymptomatic or } \\
\text { symptomatic without } \\
\text { visual impairment (i.e., } \\
\text { pain and irritation) }\end{array}$ & $\begin{array}{l}\text { symptomatic and } \\
\text { interfering with } \\
\text { function, but not } \\
\text { interfering with } \\
\text { activities of daily living }\end{array}$ & $\begin{array}{l}\text { symptomatic and } \\
\text { interfering with } \\
\text { activities of daily living }\end{array}$ & - \\
\hline Dry eye & normal & $\begin{array}{l}\text { mild, not requiring } \\
\text { treatment }\end{array}$ & $\begin{array}{l}\text { moderate or requiring } \\
\text { artificial tears }\end{array}$ & - & - \\
\hline Glaucoma & none & $\begin{array}{l}\text { increase in intraocular } \\
\text { pressure but no visual } \\
\text { loss }\end{array}$ & $\begin{array}{l}\text { increase in intraocular } \\
\text { pressure with retinal } \\
\text { changes }\end{array}$ & visual impairment & $\begin{array}{l}\text { unilateral or bilateral } \\
\text { loss of vision } \\
\text { (blindness) }\end{array}$ \\
\hline $\begin{array}{l}\text { Keratitis } \\
\text { (corneal inflammation/ } \\
\text { corneal ulceration) }\end{array}$ & none & $\begin{array}{l}\text { abnormal } \\
\text { ophthalmologic changes } \\
\text { but asymptomatic or } \\
\text { symptomatic without } \\
\text { visual impairment (i.e., } \\
\text { pain and irritation) }\end{array}$ & $\begin{array}{l}\text { symptomatic and } \\
\text { interfering with } \\
\text { function, but not } \\
\text { interfering with } \\
\text { activities of daily living }\end{array}$ & $\begin{array}{l}\text { symptomatic and } \\
\text { interfering with } \\
\text { activities of daily living }\end{array}$ & $\begin{array}{l}\text { unilateral or bilateral } \\
\text { loss of vision } \\
\text { (blindness) }\end{array}$ \\
\hline Tearing (watery eyes) & none & $\begin{array}{l}\text { mild: not interfering } \\
\text { with function }\end{array}$ & $\begin{array}{l}\text { moderate: interfering } \\
\text { with function, but not } \\
\text { interfering with } \\
\text { activities of daily living }\end{array}$ & $\begin{array}{l}\text { interfering with } \\
\text { activities of daily living }\end{array}$ & - \\
\hline Vision-blurred vision & normal & - & $\begin{array}{l}\text { symptomatic and } \\
\text { interfering with } \\
\text { function, but not } \\
\text { interfering with } \\
\text { activities of daily living }\end{array}$ & $\begin{array}{l}\text { symptomatic and } \\
\text { interfering with } \\
\text { activities of daily living }\end{array}$ & - \\
\hline $\begin{array}{l}\text { Vision-double vision } \\
\text { (diplopia) }\end{array}$ & normal & - & $\begin{array}{l}\text { symptomatic and } \\
\text { interfering with } \\
\text { function, but not } \\
\text { interfering with } \\
\text { activities of daily living }\end{array}$ & $\begin{array}{l}\text { symptomatic and } \\
\text { interfering with } \\
\text { activities of daily living }\end{array}$ & - \\
\hline $\begin{array}{l}\text { Vision-flashing } \\
\text { lights/floaters }\end{array}$ & normal & $\begin{array}{l}\text { mild, not interfering } \\
\text { with function }\end{array}$ & $\begin{array}{l}\text { symptomatic and } \\
\text { interfering with } \\
\text { function, but not } \\
\text { interfering with } \\
\text { activities of daily living }\end{array}$ & $\begin{array}{l}\text { symptomatic and } \\
\text { interfering with } \\
\text { activities of daily living }\end{array}$ & - \\
\hline
\end{tabular}


CTC Version 2.0

Publish Date: April 30, 1999

\begin{tabular}{|c|c|c|c|c|c|}
\hline \multicolumn{6}{|c|}{ Grade } \\
\hline Adverse Event & $\mathbf{0}$ & $\mathbf{1}$ & 2 & 3 & 4 \\
\hline $\begin{array}{l}\text { Vision-night blindness } \\
\text { (nyctalopia) }\end{array}$ & normal & $\begin{array}{l}\text { abnormal electro- } \\
\text { retinography but } \\
\text { asymptomatic }\end{array}$ & $\begin{array}{l}\text { symptomatic and } \\
\text { interfering with } \\
\text { function, but not } \\
\text { interfering with } \\
\text { activities of daily living }\end{array}$ & $\begin{array}{l}\text { symptomatic and } \\
\text { interfering with } \\
\text { activities of daily living }\end{array}$ & - \\
\hline Vision-photophobia & normal & - & $\begin{array}{l}\text { symptomatic and } \\
\text { interfering with } \\
\text { function, but not } \\
\text { interfering with } \\
\text { activities of daily living }\end{array}$ & $\begin{array}{l}\text { symptomatic and } \\
\text { interfering with } \\
\text { activities of daily living }\end{array}$ & - \\
\hline $\begin{array}{l}\text { Ocular/Visual - Other } \\
\text { (Specify, }\end{array}$ & normal & mild & moderate & severe & $\begin{array}{l}\text { unilateral or bilateral } \\
\text { loss of vision } \\
\text { (blindness) }\end{array}$ \\
\hline \multicolumn{6}{|c|}{ PAIN } \\
\hline Abdominal pain or cramping & none & $\begin{array}{l}\text { mild pain not interfering } \\
\text { with function }\end{array}$ & $\begin{array}{l}\text { moderate pain: pain or } \\
\text { analgesics interfering } \\
\text { with function, but not } \\
\text { interfering with } \\
\text { activities of daily living }\end{array}$ & $\begin{array}{l}\text { severe pain: pain or } \\
\text { analgesics severely } \\
\text { interfering with } \\
\text { activities of daily living }\end{array}$ & disabling \\
\hline $\begin{array}{l}\text { Arthralgia } \\
\text { (joint pain) }\end{array}$ & none & $\begin{array}{l}\text { mild pain not interfering } \\
\text { with function }\end{array}$ & $\begin{array}{l}\text { moderate pain: pain or } \\
\text { analgesics interfering } \\
\text { with function, but not } \\
\text { interfering with } \\
\text { activities of daily living }\end{array}$ & $\begin{array}{l}\text { severe pain: pain or } \\
\text { analgesics severely } \\
\text { interfering with } \\
\text { activities of daily living }\end{array}$ & disabling \\
\hline \multicolumn{6}{|c|}{ Arthritis (joint pain with clinical signs of inflammation) is graded in the MUSCULOSKELETAL category. } \\
\hline Bone pain & none & $\begin{array}{l}\text { mild pain not interfering } \\
\text { with function }\end{array}$ & $\begin{array}{l}\text { moderate pain: pain or } \\
\text { analgesics interfering } \\
\text { with function, but not } \\
\text { interfering with } \\
\text { activities of daily living }\end{array}$ & $\begin{array}{l}\text { severe pain: pain or } \\
\text { analgesics severely } \\
\text { interfering with } \\
\text { activities of daily living }\end{array}$ & disabling \\
\hline $\begin{array}{l}\text { Chest pain } \\
\text { (non-cardiac and non- } \\
\text { pleuritic) }\end{array}$ & none & $\begin{array}{l}\text { mild pain not interfering } \\
\text { with function }\end{array}$ & $\begin{array}{l}\text { moderate pain: pain or } \\
\text { analgesics interfering } \\
\text { with function, but not } \\
\text { interfering with } \\
\text { activities of daily living }\end{array}$ & $\begin{array}{l}\text { severe pain: pain or } \\
\text { analgesics severely } \\
\text { interfering with } \\
\text { activities of daily living }\end{array}$ & disabling \\
\hline Dysmenorrhea & none & $\begin{array}{l}\text { mild pain not interfering } \\
\text { with function }\end{array}$ & $\begin{array}{l}\text { moderate pain: pain or } \\
\text { analgesics interfering } \\
\text { with function, but not } \\
\text { interfering with } \\
\text { activities of daily living }\end{array}$ & $\begin{array}{l}\text { severe pain: pain or } \\
\text { analgesics severely } \\
\text { interfering with } \\
\text { activities of daily living }\end{array}$ & disabling \\
\hline Dyspareunia & none & $\begin{array}{l}\text { mild pain not interfering } \\
\text { with function }\end{array}$ & $\begin{array}{l}\text { moderate pain } \\
\text { interfering with sexual } \\
\text { activity }\end{array}$ & $\begin{array}{l}\text { severe pain preventing } \\
\text { sexual activity }\end{array}$ & - \\
\hline \multicolumn{6}{|c|}{ Dysuria is graded in the RENAL/GENITOURINARY category. } \\
\hline Earache (otalgia) & none & $\begin{array}{l}\text { mild pain not interfering } \\
\text { with function }\end{array}$ & $\begin{array}{l}\text { moderate pain: pain or } \\
\text { analgesics interfering } \\
\text { with function, but not } \\
\text { interfering with } \\
\text { activities of daily living }\end{array}$ & $\begin{array}{l}\text { severe pain: pain or } \\
\text { analgesics severely } \\
\text { interfering with } \\
\text { activities of daily living }\end{array}$ & disabling \\
\hline Headache & none & $\begin{array}{l}\text { mild pain not interfering } \\
\text { with function }\end{array}$ & $\begin{array}{l}\text { moderate pain: pain or } \\
\text { analgesics interfering } \\
\text { with function, but not } \\
\text { interfering with } \\
\text { activities of daily living }\end{array}$ & $\begin{array}{l}\text { severe pain: pain or } \\
\text { analgesics severely } \\
\text { interfering with } \\
\text { activities of daily living }\end{array}$ & disabling \\
\hline
\end{tabular}


CTC Version 2.0

Publish Date: April 30, 1999

\begin{tabular}{|c|c|c|c|c|c|}
\hline \multicolumn{6}{|c|}{ Grade } \\
\hline Adverse Event & $\mathbf{0}$ & 1 & 2 & 3 & 4 \\
\hline Hepatic pain & none & $\begin{array}{l}\text { mild pain not interfering } \\
\text { with function }\end{array}$ & $\begin{array}{l}\text { moderate pain: pain or } \\
\text { analgesics interfering } \\
\text { with function, but not } \\
\text { interfering with } \\
\text { activities of daily living }\end{array}$ & $\begin{array}{l}\text { severe pain: pain or } \\
\text { analgesics severely } \\
\text { interfering with } \\
\text { activities of daily living }\end{array}$ & disabling \\
\hline $\begin{array}{l}\text { Myalgia } \\
\text { (muscle pain) }\end{array}$ & none & $\begin{array}{l}\text { mild pain not interfering } \\
\text { with function }\end{array}$ & $\begin{array}{l}\text { moderate pain: pain or } \\
\text { analgesics interfering } \\
\text { with function, but not } \\
\text { interfering with } \\
\text { activities of daily living }\end{array}$ & $\begin{array}{l}\text { severe pain: pain or } \\
\text { analgesics severely } \\
\text { interfering with } \\
\text { activities of daily living }\end{array}$ & disabling \\
\hline $\begin{array}{l}\text { Neuropathic pain } \\
\text { (e.g., jaw pain, neurologic } \\
\text { pain, phantom limb pain, } \\
\text { post-infectious neuralgia, or } \\
\text { painful neuropathies) }\end{array}$ & none & $\begin{array}{l}\text { mild pain not interfering } \\
\text { with function }\end{array}$ & $\begin{array}{l}\text { moderate pain: pain or } \\
\text { analgesics interfering } \\
\text { with function, but not } \\
\text { interfering with } \\
\text { activities of daily living }\end{array}$ & $\begin{array}{l}\text { severe pain: pain or } \\
\text { analgesics severely } \\
\text { interfering with } \\
\text { activities of daily living }\end{array}$ & disabling \\
\hline Pain due to radiation & none & $\begin{array}{l}\text { mild pain not interfering } \\
\text { with function }\end{array}$ & $\begin{array}{l}\text { moderate pain: pain or } \\
\text { analgesics interfering } \\
\text { with function, but not } \\
\text { interfering with } \\
\text { activities of daily living }\end{array}$ & $\begin{array}{l}\text { severe pain: pain or } \\
\text { analgesics severely } \\
\text { interfering with } \\
\text { activities of daily living }\end{array}$ & disabling \\
\hline Pelvic pain & none & $\begin{array}{l}\text { mild pain not interfering } \\
\text { with function }\end{array}$ & $\begin{array}{l}\text { moderate pain: pain or } \\
\text { analgesics interfering } \\
\text { with function, but not } \\
\text { interfering with } \\
\text { activities of daily living }\end{array}$ & $\begin{array}{l}\text { severe pain: pain or } \\
\text { analgesics severely } \\
\text { interfering with } \\
\text { activities of daily living }\end{array}$ & disabling \\
\hline Pleuritic pain & none & $\begin{array}{l}\text { mild pain not interfering } \\
\text { with function }\end{array}$ & $\begin{array}{l}\text { moderate pain: pain or } \\
\text { analgesics interfering } \\
\text { with function, but not } \\
\text { interfering with } \\
\text { activities of daily living }\end{array}$ & $\begin{array}{l}\text { severe pain: pain or } \\
\text { analgesics severely } \\
\text { interfering with } \\
\text { activities of daily living }\end{array}$ & disabling \\
\hline $\begin{array}{l}\text { Rectal or perirectal pain } \\
\text { (proctalgia) }\end{array}$ & none & $\begin{array}{l}\text { mild pain not interfering } \\
\text { with function }\end{array}$ & $\begin{array}{l}\text { moderate pain: pain or } \\
\text { analgesics interfering } \\
\text { with function, but not } \\
\text { interfering with } \\
\text { activities of daily living }\end{array}$ & $\begin{array}{l}\text { severe pain: pain or } \\
\text { analgesics severely } \\
\text { interfering with } \\
\text { activities of daily living }\end{array}$ & disabling \\
\hline $\begin{array}{l}\text { Tumor pain } \\
\text { (onset or exacerbation of } \\
\text { tumor pain due to treatment) }\end{array}$ & none & $\begin{array}{l}\text { mild pain not interfering } \\
\text { with function }\end{array}$ & $\begin{array}{l}\text { moderate pain: pain or } \\
\text { analgesics interfering } \\
\text { with function, but not } \\
\text { interfering with } \\
\text { activities of daily living }\end{array}$ & $\begin{array}{l}\text { severe pain: pain or } \\
\text { analgesics severely } \\
\text { interfering with } \\
\text { activities of daily living }\end{array}$ & disabling \\
\hline \multicolumn{6}{|c|}{ Tumor flare is graded in the SYNDROME category. } \\
\hline $\begin{array}{l}\text { Pain - Other } \\
\text { (Specify, }\end{array}$ & none & mild & moderate & severe & disabling \\
\hline \multicolumn{6}{|c|}{ PULMONARY } \\
\hline $\begin{array}{l}\text { Adult Respiratory Distress } \\
\text { Syndrome (ARDS) }\end{array}$ & absent & - & - & - & present \\
\hline Apnea & none & - & - & present & requiring intubation \\
\hline
\end{tabular}


CTC Version 2.0

Publish Date: April 30, 1999

\begin{tabular}{|c|c|c|c|c|c|}
\hline \multicolumn{6}{|c|}{ Grade } \\
\hline Adverse Event & $\mathbf{0}$ & 1 & 2 & 3 & 4 \\
\hline $\begin{array}{l}\text { Carbon monoxide diffusion } \\
\text { capacity }\left(\mathrm{DL}_{\mathrm{CO}}\right)\end{array}$ & $\begin{array}{l}\geq 90 \% \text { of } \\
\text { pretreatment or } \\
\text { normal value }\end{array}$ & $\begin{array}{l}\geq 75-<90 \% \text { of } \\
\text { pretreatment or normal } \\
\text { value }\end{array}$ & $\begin{array}{l}\geq 50-<75 \% \text { of } \\
\text { pretreatment or normal } \\
\text { value }\end{array}$ & $\begin{array}{l}\geq 25-<50 \% \text { of } \\
\text { pretreatment or normal } \\
\text { value }\end{array}$ & $\begin{array}{l}<25 \% \text { of pretreatment } \\
\text { or normal value }\end{array}$ \\
\hline Cough & absent & $\begin{array}{l}\text { mild, relieved by non- } \\
\text { prescription medication }\end{array}$ & $\begin{array}{l}\text { requiring narcotic } \\
\text { antitussive }\end{array}$ & $\begin{array}{l}\text { severe cough or } \\
\text { coughing spasms, } \\
\text { poorly controlled or } \\
\text { unresponsive to } \\
\text { treatment }\end{array}$ & - \\
\hline $\begin{array}{l}\text { Dyspnea } \\
\text { (shortness of breath) }\end{array}$ & normal & - & dyspnea on exertion & $\begin{array}{l}\text { dyspnea at normal level } \\
\text { of activity }\end{array}$ & $\begin{array}{l}\text { dyspnea at rest or } \\
\text { requiring ventilator } \\
\text { support }\end{array}$ \\
\hline $\mathrm{FEV}_{1}$ & $\begin{array}{l}\geq 90 \% \text { of } \\
\text { pretreatment or } \\
\text { normal value }\end{array}$ & $\begin{array}{l}\geq 75-<90 \% \text { of } \\
\text { pretreatment or normal } \\
\text { value }\end{array}$ & $\begin{array}{l}\geq 50-<75 \% \text { of } \\
\text { pretreatment or normal } \\
\text { value }\end{array}$ & $\begin{array}{l}\geq 25-<50 \% \text { of } \\
\text { pretreatment or normal } \\
\text { value }\end{array}$ & $\begin{array}{l}<25 \% \text { of pretreatment } \\
\text { or normal value }\end{array}$ \\
\hline $\begin{array}{l}\text { Hiccoughs (hiccups, } \\
\text { singultus) }\end{array}$ & none & $\begin{array}{l}\text { mild, not requiring } \\
\text { treatment }\end{array}$ & $\begin{array}{l}\text { moderate, requiring } \\
\text { treatment }\end{array}$ & $\begin{array}{l}\text { severe, prolonged, and } \\
\text { refractory to treatment }\end{array}$ & - \\
\hline Hypoxia & normal & - & $\begin{array}{l}\text { decreased } \mathrm{O}_{2} \text { saturation } \\
\text { with exercise }\end{array}$ & $\begin{array}{l}\text { decreased } \mathrm{O}_{2} \text { saturation } \\
\text { at rest, requiring } \\
\text { supplemental oxygen }\end{array}$ & $\begin{array}{l}\text { decreased } \mathrm{O}_{2} \text { saturation, } \\
\text { requiring pressure } \\
\text { support (CPAP) or } \\
\text { assisted ventilation }\end{array}$ \\
\hline $\begin{array}{l}\text { Pleural effusion } \\
\text { (non-malignant) }\end{array}$ & none & $\begin{array}{l}\text { asymptomatic and not } \\
\text { requiring treatment }\end{array}$ & $\begin{array}{l}\text { symptomatic, requiring } \\
\text { diuretics }\end{array}$ & $\begin{array}{l}\text { symptomatic, requiring } \\
\mathrm{O}_{2} \text { or therapeutic } \\
\text { thoracentesis }\end{array}$ & $\begin{array}{l}\text { life-threatening (e.g., } \\
\text { requiring intubation) }\end{array}$ \\
\hline \multicolumn{6}{|c|}{ Pleuritic pain is graded in the PAIN category. } \\
\hline $\begin{array}{l}\text { Pneumonitis/pulmonary } \\
\text { infiltrates }\end{array}$ & none & $\begin{array}{l}\text { radiographic changes } \\
\text { but asymptomatic or } \\
\text { symptoms not requiring } \\
\text { steroids }\end{array}$ & $\begin{array}{l}\text { radiographic changes } \\
\text { and requiring steroids or } \\
\text { diuretics }\end{array}$ & $\begin{array}{l}\text { radiographic changes } \\
\text { and requiring oxygen }\end{array}$ & $\begin{array}{l}\text { radiographic changes } \\
\text { and requiring assisted } \\
\text { ventilation }\end{array}$ \\
\hline Pneumothorax & none & no intervention required & chest tube required & $\begin{array}{l}\text { sclerosis or surgery } \\
\text { required }\end{array}$ & life-threatening \\
\hline \multicolumn{6}{|c|}{ Pulmonary embolism is graded as Thrombosis/embolism in the CARDIOVASCULAR (GENERAL) category. } \\
\hline Pulmonary fibrosis & none & $\begin{array}{l}\text { radiographic changes, } \\
\text { but asymptomatic or } \\
\text { symptoms not requiring } \\
\text { steroids }\end{array}$ & $\begin{array}{l}\text { requiring steroids or } \\
\text { diuretics }\end{array}$ & requiring oxygen & $\begin{array}{l}\text { requiring assisted } \\
\text { ventilation }\end{array}$ \\
\hline $\begin{array}{l}\text { Voice changes/stridor/larynx } \\
\text { (e.g., hoarseness, loss of } \\
\text { voice, laryngitis) }\end{array}$ & normal & $\begin{array}{l}\text { mild or intermittent } \\
\text { hoarseness }\end{array}$ & $\begin{array}{l}\text { persistent hoarseness, } \\
\text { but able to vocalize; } \\
\text { may have mild to } \\
\text { moderate edema }\end{array}$ & $\begin{array}{l}\text { whispered speech, not } \\
\text { able to vocalize; may } \\
\text { have marked edema }\end{array}$ & $\begin{array}{l}\text { marked dyspnea/stridor } \\
\text { requiring tracheostomy } \\
\text { or intubation }\end{array}$ \\
\hline \multicolumn{6}{|c|}{ Notes: Cough from radiation is graded as cough in the PULMONARY category. } \\
\hline \multicolumn{6}{|c|}{$\begin{array}{l}\text { Radiation-related hemoptysis from larynx/pharynx is graded as Grade } 4 \text { Mucositis due to radiation in the GASTROINTESTINAL category. Radiation- } \\
\text { related hemoptysis from the thoracic cavity is graded as Grade } 4 \text { Hemoptysis in the HEMORRHAGE category. }\end{array}$} \\
\hline $\begin{array}{l}\text { Pulmonary - Other } \\
\text { (Specify, }\end{array}$ & none & mild & moderate & severe & $\begin{array}{l}\text { life-threatening or } \\
\text { disabling }\end{array}$ \\
\hline
\end{tabular}


CTC Version 2.0

Publish Date: April 30, 1999

\begin{tabular}{|c|c|c|c|c|c|}
\hline \multicolumn{6}{|c|}{ Grade } \\
\hline Adverse Event & $\mathbf{0}$ & $\mathbf{1}$ & 2 & 3 & 4 \\
\hline \multicolumn{6}{|c|}{ RENAL/GENITOURINARY } \\
\hline Bladder spasms & absent & $\begin{array}{l}\text { mild symptoms, not } \\
\text { requiring intervention }\end{array}$ & $\begin{array}{l}\text { symptoms requiring } \\
\text { antispasmodic }\end{array}$ & $\begin{array}{l}\text { severe symptoms } \\
\text { requiring narcotic }\end{array}$ & - \\
\hline $\begin{array}{l}\text { Creatinine } \\
\text { Note: Adjust to age-appropric }\end{array}$ & $\begin{array}{l}\text { WNL } \\
\text { e levels for pediatr }\end{array}$ & $\begin{array}{l}>\mathrm{ULN}-1.5 \mathrm{x} \text { ULN } \\
\text { tients. }\end{array}$ & $>1.5-3.0 \times$ ULN & $>3.0-6.0 \times$ ULN & $>6.0 \times \mathrm{ULN}$ \\
\hline $\begin{array}{l}\text { Dysuria } \\
\text { (painful urination) }\end{array}$ & none & $\begin{array}{l}\text { mild symptoms } \\
\text { requiring no } \\
\text { intervention }\end{array}$ & $\begin{array}{l}\text { symptoms relieved with } \\
\text { therapy }\end{array}$ & $\begin{array}{l}\text { symptoms not relieved } \\
\text { despite therapy }\end{array}$ & - \\
\hline $\begin{array}{l}\text { Fistula or GU fistula } \\
\text { (e.g., vaginal, vesicovaginal) }\end{array}$ & none & - & - & requiring intervention & requiring surgery \\
\hline Hemoglobinuria & - & present & - & - & - \\
\hline \multicolumn{6}{|c|}{ Hematuria (in the absence of vaginal bleeding) is graded in the HEMORRHAGE category. } \\
\hline Incontinence & none & $\begin{array}{l}\text { with coughing, } \\
\text { sneezing, etc. }\end{array}$ & $\begin{array}{l}\text { spontaneous, some } \\
\text { control }\end{array}$ & $\begin{array}{l}\text { no control (in the } \\
\text { absence of fistula) }\end{array}$ & - \\
\hline $\begin{array}{l}\text { Operative injury to bladder } \\
\text { and/or ureter }\end{array}$ & none & - & $\begin{array}{l}\text { injury of bladder with } \\
\text { primary repair }\end{array}$ & $\begin{array}{l}\text { sepsis, fistula, or } \\
\text { obstruction requiring } \\
\text { secondary surgery; loss } \\
\text { of one kidney; injury } \\
\text { requiring anastomosis } \\
\text { or re-implantation }\end{array}$ & $\begin{array}{l}\text { septic obstruction of } \\
\text { both kidneys or } \\
\text { vesicovaginal fistula } \\
\text { requiring diversion }\end{array}$ \\
\hline Proteinuria & $\begin{array}{l}\text { normal or }<0.15 \\
\mathrm{~g} / 24 \text { hours }\end{array}$ & $\begin{array}{l}1+\text { or } 0.15-1.0 \mathrm{~g} / 24 \\
\text { hours }\end{array}$ & $\begin{array}{l}2+\text { to } 3+\text { or } 1.0-3.5 \\
\mathrm{~g} / 24 \text { hours }\end{array}$ & $4+$ or $>3.5 \mathrm{~g} / 24$ hours & nephrotic syndrome \\
\hline Renal failure & none & - & - & $\begin{array}{l}\text { requiring dialysis, but } \\
\text { reversible }\end{array}$ & $\begin{array}{l}\text { requiring dialysis and } \\
\text { irreversible }\end{array}$ \\
\hline Ureteral obstruction & none & $\begin{array}{l}\text { unilateral, not requiring } \\
\text { surgery }\end{array}$ & - & $\begin{array}{l}\text { bilateral, not requiring } \\
\text { surgery }\end{array}$ & $\begin{array}{l}\text { stent, nephrostomy } \\
\text { tube, or surgery }\end{array}$ \\
\hline $\begin{array}{l}\text { Urinary electrolyte wasting } \\
\text { (e.g., Fanconi's syndrome, } \\
\text { renal tubular acidosis) }\end{array}$ & none & $\begin{array}{l}\text { asymptomatic, not } \\
\text { requiring treatment }\end{array}$ & $\begin{array}{l}\text { mild, reversible and } \\
\text { manageable with oral } \\
\text { replacement }\end{array}$ & $\begin{array}{l}\text { reversible but requiring } \\
\text { IV replacement }\end{array}$ & $\begin{array}{l}\text { irreversible, requiring } \\
\text { continued replacement }\end{array}$ \\
\hline Urinary frequency/urgency & normal & $\begin{array}{l}\text { increase in frequency or } \\
\text { nocturia up to } 2 \mathrm{x} \\
\text { normal }\end{array}$ & $\begin{array}{l}\text { increase }>2 \text { x normal } \\
\text { but }<\text { hourly }\end{array}$ & $\begin{array}{l}\text { hourly or more with } \\
\text { urgency, or requiring } \\
\text { catheter }\end{array}$ & - \\
\hline Urinary retention & normal & $\begin{array}{l}\text { hesitancy or dribbling, } \\
\text { but no significant } \\
\text { residual urine; retention } \\
\text { occurring during the } \\
\text { immediate } \\
\text { postoperative period }\end{array}$ & $\begin{array}{l}\text { hesitancy requiring } \\
\text { medication or } \\
\text { occasional in/out } \\
\text { catheterization }(<4 \mathrm{x} \text { per } \\
\text { week }) \text {, or operative } \\
\text { bladder atony requiring } \\
\text { indwelling catheter } \\
\text { beyond immediate } \\
\text { postoperative period but } \\
\text { for }<6 \text { weeks }\end{array}$ & $\begin{array}{l}\text { requiring frequent } \\
\text { in/out catheterization } \\
\text { ( } \geq 4 \times \text { per week) or } \\
\text { urological intervention } \\
\text { (e.g., TURP, suprapubic } \\
\text { tube, urethrotomy) }\end{array}$ & bladder rupture \\
\hline
\end{tabular}




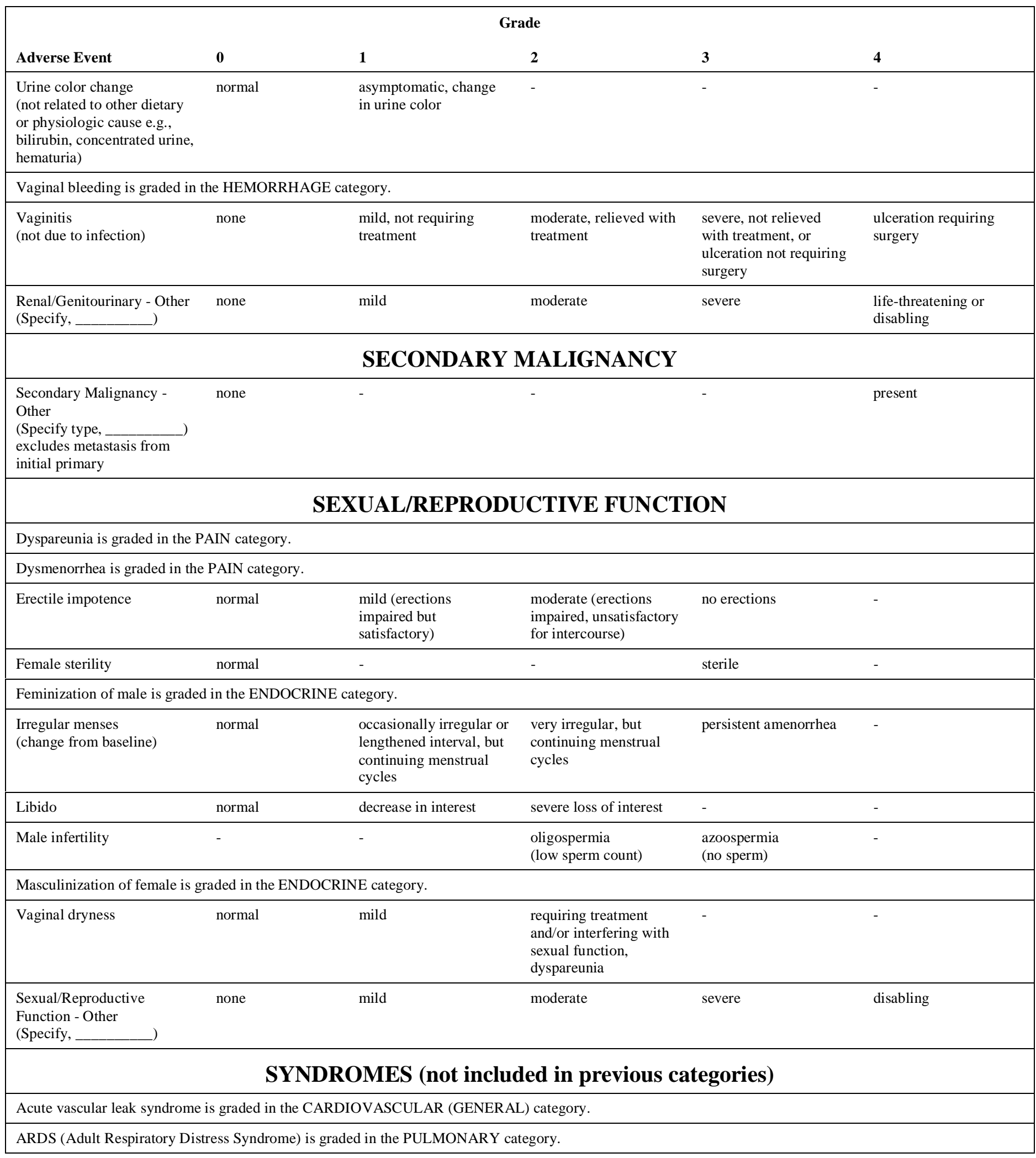


CTC Version 2.0

Publish Date: April 30, 1999

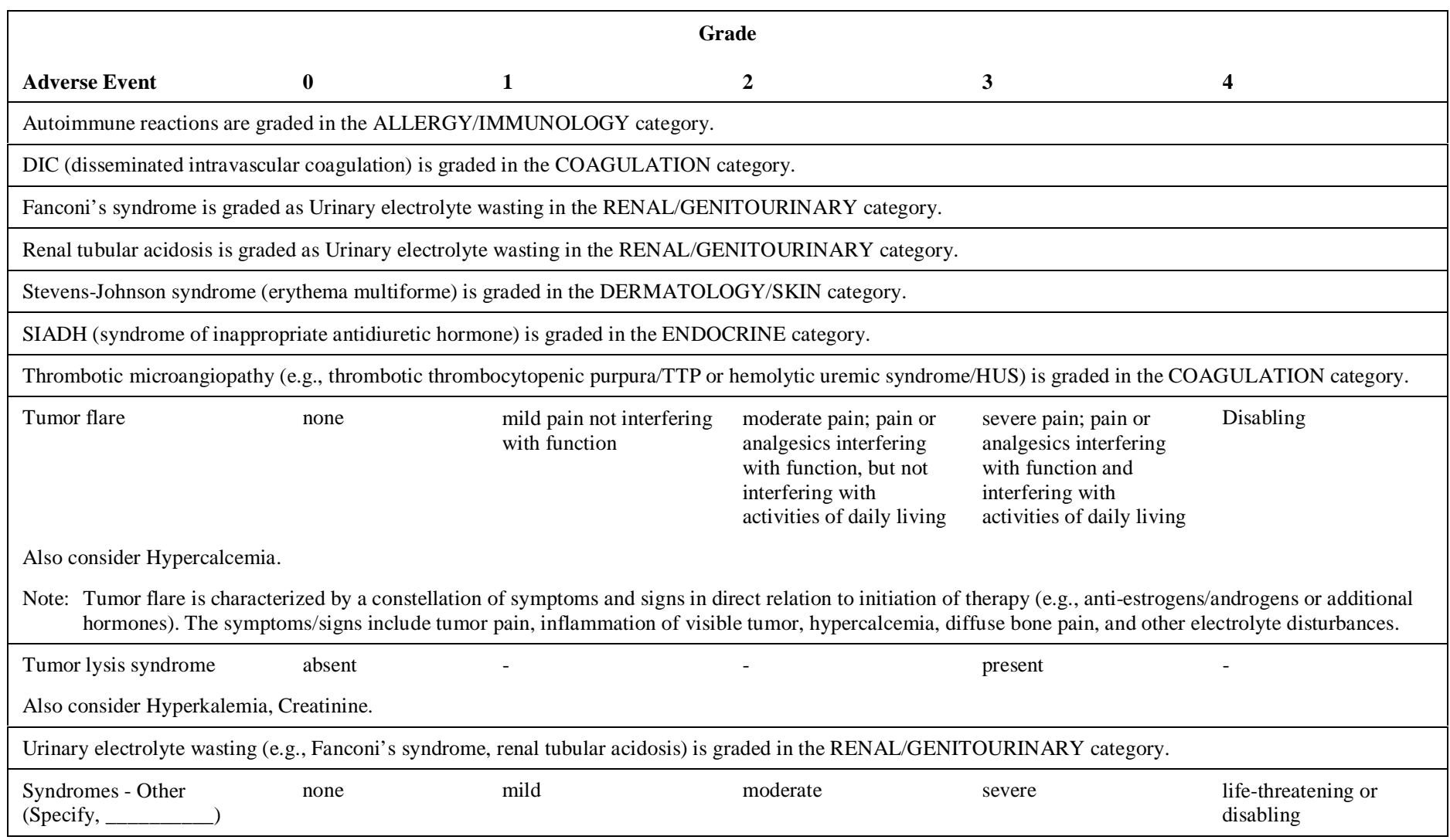




\section{Appendix I}

\section{Adverse Event Module}

To be implemented at the request of the study sponsor or principal investigator in the protocol or by protocol amendment when more detailed information is considered pertinent.

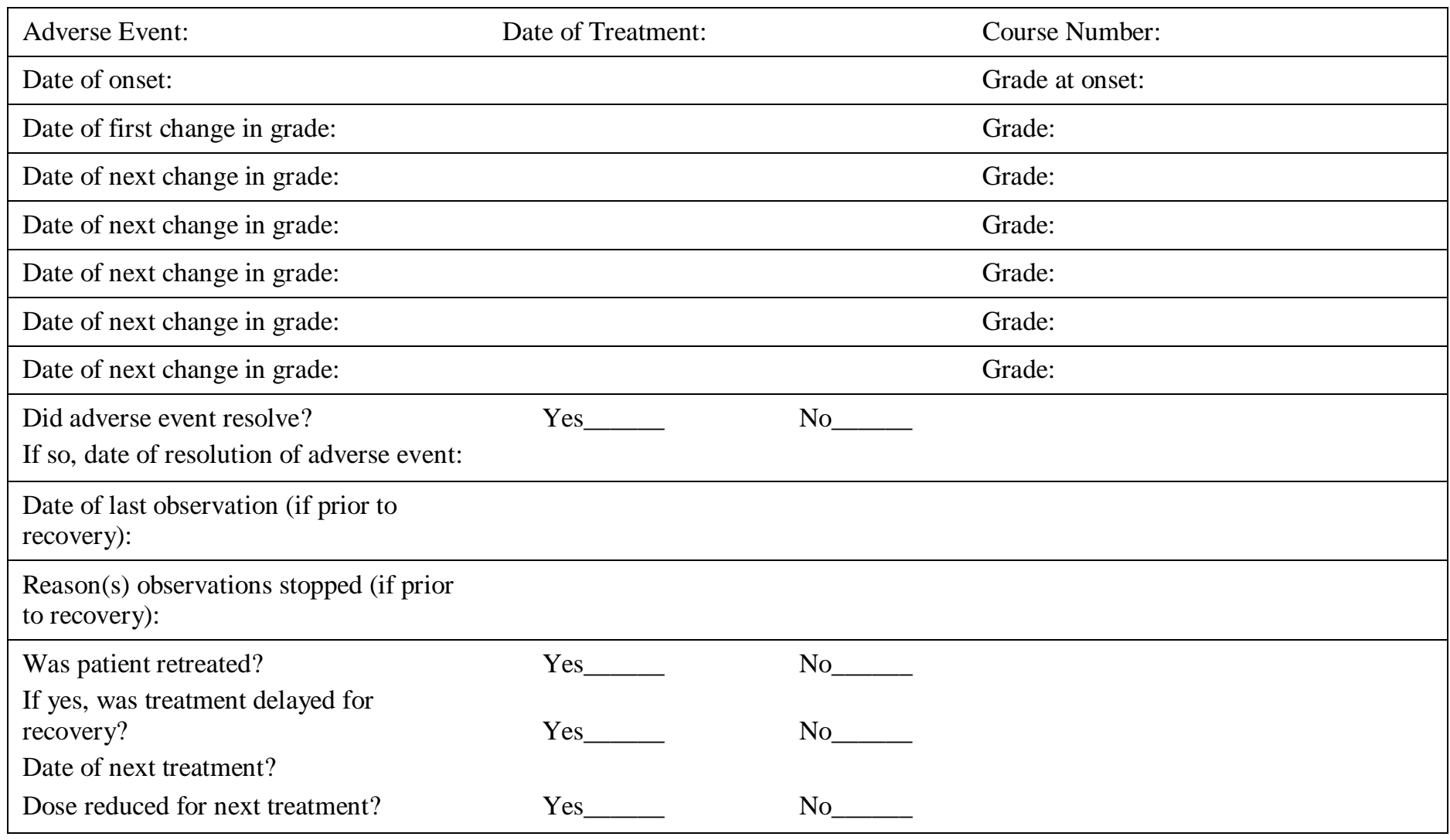

Additional Comments:

If module is being activated for new adverse event not currently in CTC, please provide definitions for adverse event grading:

Grade $0=$

Grade $1=$

Grade 2 =

Grade 3 =

Grade $4=$ 


\section{Appendix II \\ Infection Module}

To be implemented at the request of the study sponsor or principal investigator in the protocol or by protocol amendment when more detailed information is considered pertinent.

1. Use the Common Toxicity Criteria definitions to grade the severity of the infection.

2. Specify type of infection from the following (CHOOSE ONE):
BACTERIAL
FUNGAL
PROTOZOAL
VIRAL
UNKNOWN

3. Specify site of infection from the following (CHOOSE ALL THAT APPLY):

BLOOD CULTURE POSITIVE

BONE INFECTION

CATHETER (intravenous)

CATHETER (intravenous), tunnel infection

CENTRAL NERVOUS SYSTEM INFECTION

EAR INFECTION

EYE INFECTION

GASTROINTESTINAL INFECTION

ORAL INFECTION

PNEUMONIA

SKIN INFECTION

UPPER RESPIRATORY INFECTION

URINARY TRACT INFECTION

VAGINAL INFECTION

INFECTION, not otherwise specified (Specify site,

4. Specify organism, if known:

5. Prophylactic antibiotic, antifungal, or antiviral therapy administration

Yes

No

If prophylaxis was given prior to infection, please specify below:

Antibiotic prophylaxis

Antifungal prophylaxis

Antiviral prophylaxis

Other prophylaxis 


\section{Appendix III}

\section{Performance Status Scales/Scores}

\section{PERFORMANCE STATUS CRITERIA}

Karnofsky and Lansky performance scores are intended to be multiples of 10 .

\begin{tabular}{|c|c|c|c|c|c|}
\hline \multicolumn{2}{|r|}{ ECOG (Zubrod) } & \multicolumn{2}{|r|}{ Karnofsky } & \multicolumn{2}{|r|}{ Lansky* } \\
\hline Score & Description & Score & Description & Score & Description \\
\hline \multirow{3}{*}{0} & \multirow{3}{*}{$\begin{array}{l}\text { Fully active, able to carry on } \\
\text { all pre-disease performance } \\
\text { without restriction. }\end{array}$} & 100 & $\begin{array}{l}\text { Normal, no complaints, no } \\
\text { evidence of disease. }\end{array}$ & 100 & Fully active, normal. \\
\hline & & & & & \\
\hline & & 90 & $\begin{array}{l}\text { Able to carry on normal } \\
\text { activity; minor signs or } \\
\text { symptoms of disease. }\end{array}$ & 90 & $\begin{array}{l}\text { Minor restrictions in physically } \\
\text { strenuous activity. }\end{array}$ \\
\hline \multirow[t]{2}{*}{1} & \multirow{2}{*}{$\begin{array}{l}\text { Restricted in physically } \\
\text { strenuous activity but } \\
\text { ambulatory and able to carry } \\
\text { out work of a light or } \\
\text { sedentary nature, e.g., light } \\
\text { housework, office work. }\end{array}$} & 80 & $\begin{array}{l}\text { Normal activity with effort; } \\
\text { some signs or symptoms of } \\
\text { disease. }\end{array}$ & 80 & Active, but tires more quickly \\
\hline & & 70 & $\begin{array}{l}\text { Cares for self, unable to carry } \\
\text { on normal activity or do active } \\
\text { work. }\end{array}$ & 70 & $\begin{array}{l}\text { Both greater restriction of and less time } \\
\text { spent in play activity. }\end{array}$ \\
\hline \multirow[t]{2}{*}{2} & \multirow{2}{*}{$\begin{array}{l}\text { Ambulatory and capable of all } \\
\text { selfcare but unable to carry } \\
\text { out any work activities. Up } \\
\text { and about more than } 50 \% \text { of } \\
\text { waking hours. }\end{array}$} & 60 & $\begin{array}{l}\text { Requires occasional } \\
\text { assistance, but is able to care } \\
\text { for most of his/her needs. }\end{array}$ & 60 & $\begin{array}{l}\text { Up and around, but minimal active play; } \\
\text { keeps busy with quieter activities. }\end{array}$ \\
\hline & & 50 & $\begin{array}{l}\text { Requires considerable } \\
\text { assistance and frequent } \\
\text { medical care. }\end{array}$ & 50 & $\begin{array}{l}\text { Gets dressed, but lies around much of } \\
\text { the day; no active play; able to } \\
\text { participate in all quiet play and } \\
\text { activities. }\end{array}$ \\
\hline \multirow{3}{*}{3} & \multirow{3}{*}{$\begin{array}{l}\text { Capable of only limited } \\
\text { selfcare, confined to bed or } \\
\text { chair more than } 50 \% \text { of } \\
\text { waking hours. }\end{array}$} & 40 & $\begin{array}{l}\text { Disabled, requires special care } \\
\text { and assistance. }\end{array}$ & 40 & $\begin{array}{l}\text { Mostly in bed; participates in quiet } \\
\text { activities. }\end{array}$ \\
\hline & & & & & \\
\hline & & 30 & $\begin{array}{l}\text { Severely disabled, } \\
\text { hospitalization indicated. } \\
\text { Death not imminent. }\end{array}$ & 30 & $\begin{array}{l}\text { In bed; needs assistance even for quiet } \\
\text { play. }\end{array}$ \\
\hline \multirow{3}{*}{4} & \multirow{3}{*}{$\begin{array}{l}\text { Completely disabled. Cannot } \\
\text { carry on any selfcare. Totally } \\
\text { confined to bed or chair. }\end{array}$} & 20 & $\begin{array}{l}\text { Very sick, hospitalization } \\
\text { indicated. Death not imminent. }\end{array}$ & 20 & $\begin{array}{l}\text { Often sleeping; play entirely limited to } \\
\text { very passive activities. }\end{array}$ \\
\hline & & & & & \\
\hline & & 10 & $\begin{array}{l}\text { Moribund, fatal processes } \\
\text { progressing rapidly. }\end{array}$ & 10 & No play; does not get out of bed. \\
\hline
\end{tabular}

*The conversion of the Lansky to ECOG scales is intended for $\mathrm{NCl}$ reporting purposes only. 


\section{Appendix IV}

\section{RTOG/EORTC Late Radiation Morbidity Scoring Scheme}

\section{Use for adverse event occurring greater than 90 days after radiation therapy.}

\begin{tabular}{|c|c|c|c|c|c|}
\hline \multicolumn{6}{|c|}{ Grade } \\
\hline Adverse Event & $\mathbf{0}$ & 1 & 2 & 3 & 4 \\
\hline $\begin{array}{l}\text { Bladder- } \\
\text { Late RT Morbidity Scoring }\end{array}$ & $\begin{array}{l}\text { No change from } \\
\text { baseline }\end{array}$ & $\begin{array}{l}\text { Slight epithelial } \\
\text { atrophy/minor } \\
\text { telangiectasia } \\
\text { (microscopic hematuria) }\end{array}$ & $\begin{array}{l}\text { Moderate frequency/ } \\
\text { generalized } \\
\text { telangiectasia/ } \\
\text { intermittent } \\
\text { macroscopic hematuria }\end{array}$ & $\begin{array}{l}\text { Severe frequency and } \\
\text { dysuria/severe } \\
\text { generalized } \\
\text { telangiectasia (often } \\
\text { with petechiae); } \\
\text { frequent hematuria; } \\
\text { reduction in bladder } \\
\text { capacity }(<150 \mathrm{~mL})\end{array}$ & $\begin{array}{l}\text { Necrosis/contracted } \\
\text { bladder (capacity < } 100 \\
\text { mL)/severe } \\
\text { hemorrhagic cystitis }\end{array}$ \\
\hline $\begin{array}{l}\text { Bone- } \\
\text { Late RT Morbidity Scoring }\end{array}$ & $\begin{array}{l}\text { No change from } \\
\text { baseline }\end{array}$ & $\begin{array}{l}\text { Asymptomatic; no } \\
\text { growth retardation; } \\
\text { reduced bone density }\end{array}$ & $\begin{array}{l}\text { Moderate pain or } \\
\text { tenderness; growth } \\
\text { retardation; irregular } \\
\text { bone sclerosis }\end{array}$ & $\begin{array}{l}\text { Severe pain or } \\
\text { tenderness; complete } \\
\text { arrest of bone growth; } \\
\text { dense bone sclerosis }\end{array}$ & $\begin{array}{l}\text { Necrosis/ } \\
\text { spontaneous fracture }\end{array}$ \\
\hline $\begin{array}{l}\text { Brain- } \\
\text { Late RT Morbidity Scoring }\end{array}$ & $\begin{array}{l}\text { No change from } \\
\text { baseline }\end{array}$ & $\begin{array}{l}\text { Mild headache; slight } \\
\text { lethargy }\end{array}$ & $\begin{array}{l}\text { Moderate headache; } \\
\text { great lethargy }\end{array}$ & $\begin{array}{l}\text { Severe headaches; } \\
\text { severe CNS dysfunction } \\
\text { (partial loss of power or } \\
\text { dyskinesia) }\end{array}$ & $\begin{array}{l}\text { Seizures or paralysis; } \\
\text { coma }\end{array}$ \\
\hline $\begin{array}{l}\text { Esophagus- } \\
\text { Late RT Morbidity Scoring }\end{array}$ & $\begin{array}{l}\text { No change from } \\
\text { baseline }\end{array}$ & $\begin{array}{l}\text { Mild fibrosis; slight } \\
\text { difficulty in swallowing } \\
\text { solids; no pain on } \\
\text { swallowing }\end{array}$ & $\begin{array}{l}\text { Unable to take solid } \\
\text { food normally; } \\
\text { swallowing semi-solid } \\
\text { food; dilation may be } \\
\text { indicated }\end{array}$ & $\begin{array}{l}\text { Severe fibrosis; able to } \\
\text { swallow only liquids; } \\
\text { may have pain on } \\
\text { swallowing; dilation } \\
\text { required }\end{array}$ & $\begin{array}{l}\text { Necrosis/ } \\
\text { perforation; fistula }\end{array}$ \\
\hline $\begin{array}{l}\text { Eye- } \\
\text { Late RT Morbidity Scoring }\end{array}$ & $\begin{array}{l}\text { No change from } \\
\text { baseline }\end{array}$ & $\begin{array}{l}\text { Asymptomatic cataract; } \\
\text { minor corneal } \\
\text { ulceration or keratitis }\end{array}$ & $\begin{array}{l}\text { Symptomatic cataract; } \\
\text { moderate corneal } \\
\text { ulceration; minor } \\
\text { retinopathy or glaucoma }\end{array}$ & $\begin{array}{l}\text { Severe keratitis; severe } \\
\text { retinopathy or } \\
\text { detachment; severe } \\
\text { glaucoma }\end{array}$ & $\begin{array}{l}\text { Panophthalmitis; } \\
\text { blindness }\end{array}$ \\
\hline $\begin{array}{l}\text { Heart- } \\
\text { Late RT Morbidity Scoring }\end{array}$ & $\begin{array}{l}\text { No change from } \\
\text { baseline }\end{array}$ & $\begin{array}{l}\text { Asymptomatic or mild } \\
\text { symptoms; transient T } \\
\text { wave inversion and ST } \\
\text { changes; sinus } \\
\text { tachycardia }>110 \text { (at } \\
\text { rest) }\end{array}$ & $\begin{array}{l}\text { Moderate angina on } \\
\text { effort; mild pericarditis; } \\
\text { normal heart size; } \\
\text { persistent abnormal T } \\
\text { wave and ST changes; } \\
\text { low QRS }\end{array}$ & $\begin{array}{l}\text { Severe angina; } \\
\text { pericardial effusion; } \\
\text { constrictive pericarditis; } \\
\text { moderate heart failure; } \\
\text { cardiac enlargement; } \\
\text { EKG abnormalities }\end{array}$ & $\begin{array}{l}\text { Tamponade/severe heart } \\
\text { failure/severe } \\
\text { constrictive pericarditis }\end{array}$ \\
\hline $\begin{array}{l}\text { Joint- } \\
\text { Late RT Morbidity Scoring }\end{array}$ & $\begin{array}{l}\text { No change from } \\
\text { baseline }\end{array}$ & $\begin{array}{l}\text { Mild joint stiffness; } \\
\text { slight limitation of } \\
\text { movement }\end{array}$ & $\begin{array}{l}\text { Moderate stiffness; } \\
\text { intermittent or moderate } \\
\text { joint pain; moderate } \\
\text { limitation of movement }\end{array}$ & $\begin{array}{l}\text { Severe joint stiffness; } \\
\text { pain with severe } \\
\text { limitation of movement }\end{array}$ & $\begin{array}{l}\text { Necrosis/complete } \\
\text { fixation }\end{array}$ \\
\hline $\begin{array}{l}\text { Kidney- } \\
\text { Late RT Morbidity Scoring }\end{array}$ & $\begin{array}{l}\text { No change from } \\
\text { baseline }\end{array}$ & $\begin{array}{l}\text { Transient albuminuria; } \\
\text { no hypertension; mild } \\
\text { impairment of renal } \\
\text { function; urea } 25-35 \\
\mathrm{mg} \% \text {; creatinine } 1.5- \\
2.0 \mathrm{mg} \% \text {; creatinine } \\
\text { clearance }>75 \%\end{array}$ & $\begin{array}{l}\text { Persistent moderate } \\
\text { albuminuria }(2+) \text {; mild } \\
\text { hypertension; no related } \\
\text { anemia; moderate } \\
\text { impairment of renal } \\
\text { function; urea }>36 \text { - } 60 \\
\text { mg\%; creatinine } \\
\text { clearance }>50-74 \%\end{array}$ & $\begin{array}{l}\text { Severe albuminuria; } \\
\text { severe hypertension; } \\
\text { persistent anemia }(<10 \\
\text { g\%); severe renal } \\
\text { failure; urea }>60 \mathrm{mg} \% \text {; } \\
\text { creatinine }>4 \mathrm{mg} \% \text {; } \\
\text { creatinine clearance } \\
<50 \%\end{array}$ & $\begin{array}{l}\text { Malignant hypertension; } \\
\text { uremic coma/urea } \\
>100 \%\end{array}$ \\
\hline $\begin{array}{l}\text { Larynx- } \\
\text { Late RT Morbidity Scoring }\end{array}$ & $\begin{array}{l}\text { No change from } \\
\text { baseline }\end{array}$ & $\begin{array}{l}\text { Hoarseness; slight } \\
\text { arytenoid edema }\end{array}$ & $\begin{array}{l}\text { Moderate arytenoid } \\
\text { edema; chondritis }\end{array}$ & $\begin{array}{l}\text { Severe edema; severe } \\
\text { chondritis }\end{array}$ & Necrosis \\
\hline
\end{tabular}




\section{Appendix IV (continued)}

\section{RTOG/EORTC Late Radiation Morbidity Scoring Scheme}

\section{Use for adverse event occurring greater than 90 days after radiation therapy.}

\begin{tabular}{|c|c|c|c|c|c|}
\hline \multicolumn{6}{|c|}{ Grade } \\
\hline Adverse Event & $\mathbf{0}$ & 1 & 2 & 3 & 4 \\
\hline $\begin{array}{l}\text { Liver- } \\
\text { Late RT Morbidity Scoring }\end{array}$ & $\begin{array}{l}\text { No change from } \\
\text { baseline }\end{array}$ & $\begin{array}{l}\text { Mild lassitude; nausea; } \\
\text { dyspepsia; slightly } \\
\text { abnormal liver function }\end{array}$ & $\begin{array}{l}\text { Moderate symptoms; } \\
\text { some abnormal liver } \\
\text { function tests; serum } \\
\text { albumin normal }\end{array}$ & $\begin{array}{l}\text { Disabling hepatic } \\
\text { insufficiency; liver } \\
\text { function tests grossly } \\
\text { abnormal; low albumin; } \\
\text { edema or ascites }\end{array}$ & $\begin{array}{l}\text { Necrosis/hepatic coma } \\
\text { or encephalopathy }\end{array}$ \\
\hline $\begin{array}{l}\text { Lung- } \\
\text { Late RT Morbidity Scoring }\end{array}$ & $\begin{array}{l}\text { No change from } \\
\text { baseline }\end{array}$ & $\begin{array}{l}\text { Asymptomatic or mild } \\
\text { symptoms (dry cough); } \\
\text { slight radiographic } \\
\text { appearances }\end{array}$ & $\begin{array}{l}\text { Moderate symptomatic } \\
\text { fibrosis or pneumonitis } \\
\text { (severe cough); low } \\
\text { grade fever; patchy } \\
\text { radiographic } \\
\text { appearances }\end{array}$ & $\begin{array}{l}\text { Severe symptomatic } \\
\text { fibrosis or pneumonitis; } \\
\text { dense radiographic } \\
\text { changes }\end{array}$ & $\begin{array}{l}\text { Severe respiratory } \\
\text { insufficiency/ } \\
\text { continuous } \mathrm{O}_{2} / \text { assisted } \\
\text { ventilation }\end{array}$ \\
\hline $\begin{array}{l}\text { Mucous membrane- } \\
\text { Late RT Morbidity Scoring }\end{array}$ & $\begin{array}{l}\text { No change from } \\
\text { baseline }\end{array}$ & $\begin{array}{l}\text { Slight atrophy and } \\
\text { dryness }\end{array}$ & $\begin{array}{l}\text { Moderate atrophy and } \\
\text { telangiectasia; little } \\
\text { mucus }\end{array}$ & $\begin{array}{l}\text { Marked atrophy with } \\
\text { complete dryness; } \\
\text { severe telangiectasia }\end{array}$ & Ulceration \\
\hline $\begin{array}{l}\text { Salivary glands- } \\
\text { Late RT Morbidity Scoring }\end{array}$ & $\begin{array}{l}\text { No change from } \\
\text { baseline }\end{array}$ & $\begin{array}{l}\text { Slight dryness of } \\
\text { mouth; good response } \\
\text { on stimulation }\end{array}$ & $\begin{array}{l}\text { Moderate dryness of } \\
\text { mouth; poor response } \\
\text { on stimulation }\end{array}$ & $\begin{array}{l}\text { Complete dryness of } \\
\text { mouth; no response on } \\
\text { stimulation }\end{array}$ & Fibrosis \\
\hline $\begin{array}{l}\text { Skin- } \\
\text { Late RT Morbidity Scoring }\end{array}$ & $\begin{array}{l}\text { No change from } \\
\text { baseline }\end{array}$ & $\begin{array}{l}\text { Slight atrophy; } \\
\text { pigmentation change; } \\
\text { some hair loss }\end{array}$ & $\begin{array}{l}\text { Patchy atrophy; } \\
\text { moderate telangiectasia; } \\
\text { total hair loss }\end{array}$ & $\begin{array}{l}\text { Marked atrophy; gross } \\
\text { telangiectasia }\end{array}$ & Ulceration \\
\hline $\begin{array}{l}\text { Small/Large intestine- } \\
\text { Late RT Morbidity Scoring }\end{array}$ & $\begin{array}{l}\text { No change from } \\
\text { baseline }\end{array}$ & $\begin{array}{l}\text { Mild diarrhea; mild } \\
\text { cramping; bowel } \\
\text { movement } 5 \mathrm{x} \text { daily; } \\
\text { slight rectal discharge } \\
\text { or bleeding }\end{array}$ & $\begin{array}{l}\text { Moderate diarrhea and } \\
\text { colic; bowel movement } \\
>5 \mathrm{x} \text { daily; excessive } \\
\text { rectal mucus or } \\
\text { intermittent bleeding }\end{array}$ & $\begin{array}{l}\text { Obstruction or bleeding, } \\
\text { requiring surgery }\end{array}$ & $\begin{array}{l}\text { Necrosis/perforation } \\
\text { fistula }\end{array}$ \\
\hline $\begin{array}{l}\text { Spinal cord- } \\
\text { Late RT Morbidity Scoring }\end{array}$ & $\begin{array}{l}\text { No change from } \\
\text { baseline }\end{array}$ & $\begin{array}{l}\text { Mild Lhermitte's } \\
\text { syndrome }\end{array}$ & $\begin{array}{l}\text { Severe Lhermitte's } \\
\text { syndrome }\end{array}$ & $\begin{array}{l}\text { Objective neurological } \\
\text { findings at or below } \\
\text { cord level treatment }\end{array}$ & $\begin{array}{l}\text { Mono-, para-, } \\
\text { quadriplegia }\end{array}$ \\
\hline $\begin{array}{l}\text { Subcutaneous tissue- } \\
\text { Late RT Morbidity Scoring }\end{array}$ & $\begin{array}{l}\text { No change from } \\
\text { baseline }\end{array}$ & $\begin{array}{l}\text { Slight induration } \\
\text { (fibrosis) and loss of } \\
\text { subcutaneous fat }\end{array}$ & $\begin{array}{l}\text { Moderate fibrosis but } \\
\text { asymptomatic; slight } \\
\text { field contracture; }<10 \% \\
\text { linear reduction }\end{array}$ & $\begin{array}{l}\text { Severe induration and } \\
\text { loss of subcutaneous } \\
\text { tissue; field contracture } \\
>10 \% \text { linear } \\
\text { measurement }\end{array}$ & Necrosis \\
\hline $\begin{array}{l}\text { Radiation - Other } \\
\text { (Specify, }\end{array}$ & None & Mild & Moderate & Severe & $\begin{array}{l}\text { Life-threatening or } \\
\text { disabling }\end{array}$ \\
\hline
\end{tabular}




\section{Appendix V}

\section{BMT-Specific Adverse Events}

\section{Summary of BMT-Specific Adverse Events that may be used if specified by the protocol. These differ} from the standard CTC and may be more relevant to the transplant setting. They are listed here for the convenience of investigators writing transplant protocols. They are also included in the CTC document.

\begin{tabular}{|c|c|c|c|c|c|}
\hline \multicolumn{6}{|c|}{ Grade } \\
\hline Adverse Event & $\mathbf{0}$ & 1 & 2 & 3 & 4 \\
\hline $\begin{array}{l}\text { Bilirubin associated with } \\
\text { graft versus host disease for } \\
\text { BMT studies . }\end{array}$ & normal & $\geq 2-<3 \mathrm{mg} / 100 \mathrm{~mL}$ & $\geq 3-<6 \mathrm{mg} / 100 \mathrm{~mL}$ & $\geq 6-<15 \mathrm{mg} / 100 \mathrm{~mL}$ & $\geq 15 \mathrm{mg} / 100 \mathrm{~mL}$ \\
\hline $\begin{array}{l}\text { Diarrhea associated with } \\
\text { graft versus host disease } \\
\text { (GVHD) for BMT studies. }\end{array}$ & none & $\begin{array}{l}>500-\leq 1000 \mathrm{~mL} \text { of } \\
\text { diarrhea/day }\end{array}$ & $\begin{array}{l}>1000-\leq 1500 \mathrm{~mL} \text { of } \\
\text { diarrhea/day }\end{array}$ & $\begin{array}{l}>1500 \mathrm{~mL} \text { of } \\
\text { diarrhea/day }\end{array}$ & $\begin{array}{l}\text { severe abdominal pain } \\
\text { with or without ileus }\end{array}$ \\
\hline $\begin{array}{l}\text { Diarrhea for pediatric BMT } \\
\text { studies. }\end{array}$ & & $\begin{array}{l}>5-\leq 10 \mathrm{~mL} / \mathrm{kg} \text { of } \\
\text { diarrhea/day }\end{array}$ & $\begin{array}{l}>10-\leq 15 \mathrm{~mL} / \mathrm{kg} \text { of } \\
\text { diarrhea/day }\end{array}$ & $\begin{array}{l}>15 \mathrm{~mL} / \mathrm{kg} \text { of } \\
\text { diarrhea/day }\end{array}$ & - \\
\hline Hepatic enlargement & absent & - & - & present & - \\
\hline $\begin{array}{l}\text { Leukocytes (total WBC) for } \\
\text { BMT studies. }\end{array}$ & WNL & $\begin{array}{l}\geq 2.0-<3.0 \times 10^{9} / \mathrm{L} \\
\geq 2000-<3000 / \mathrm{mm}^{3}\end{array}$ & $\begin{array}{l}\geq 1.0-<2.0 \times 10^{9} / \mathrm{L} \\
\geq 1000-<2000 / \mathrm{mm}^{3}\end{array}$ & $\begin{array}{l}\geq 0.5-<1.0 \times 10^{9} / \mathrm{L} \\
\geq 500-<1000 / \mathrm{mm}^{3}\end{array}$ & $\begin{array}{l}<0.5 \times 10^{9} / \mathrm{L} \\
<500 / \mathrm{mm}^{3}\end{array}$ \\
\hline $\begin{array}{l}\text { Leukocytes (total WBC) for } \\
\text { pediatric BMT studies (using } \\
\text { age, race and sex normal } \\
\text { values). }\end{array}$ & & $\geq 75-<100 \%$ LLN & $\geq 50-<75 \% L L N$ & $\geq 25-50 \%$ LLN & $<25 \% L L N$ \\
\hline $\begin{array}{l}\text { Lymphopenia for pediatric } \\
\text { BMT studies (using age, } \\
\text { race and sex normal values). }\end{array}$ & $\mathrm{mm}^{3}$ & $\geq 75-<100 \% L L N$ & $\geq 50-<75 \% L L N$ & $\geq 25-<50 \% L L N$ & $<25 \% L L N$ \\
\hline $\begin{array}{l}\text { Neutrophils/granulocytes } \\
\text { (ANC/AGC) for BMT } \\
\text { studies. }\end{array}$ & WNL & $\begin{array}{l}\geq 1.0-<1.5 \times 10^{9} / \mathrm{L} \\
\geq 1000-<1500 / \mathrm{mm}^{3}\end{array}$ & $\begin{array}{l}\geq 0.5-<1.0 \times 10^{9} / \mathrm{L} \\
\geq 500-<1000 / \mathrm{mm}^{3}\end{array}$ & $\begin{array}{l}\geq 0.1-<0.5 \times 10^{9} / \mathrm{L} \\
\geq 100-<500 / \mathrm{mm}^{3}\end{array}$ & $\begin{array}{l}<0.1 \times 10^{9} / \mathrm{L} \\
<100 / \mathrm{mm}^{3}\end{array}$ \\
\hline Platelets for BMT studies. & WNL & $\begin{array}{l}\geq 50.0-<75.0 \times 10^{9} / \mathrm{L} \\
\geq 50,000-<75,000 / \mathrm{mm}^{3}\end{array}$ & $\begin{array}{l}\geq 20.0-<50.0 \times 10^{9} / \mathrm{L} \\
\geq 20,000-<50,000 / \mathrm{mm}^{3}\end{array}$ & $\begin{array}{l}\geq 10.0-<20.0 \times 10^{9} / \mathrm{L} \\
\geq 10,000-<20,000 / \mathrm{mm}^{3}\end{array}$ & $\begin{array}{l}<10.0 \times 10^{9} / \mathrm{L} \\
<10,000 / \mathrm{mm}^{3}\end{array}$ \\
\hline $\begin{array}{l}\text { Rash/dermatitis associated } \\
\text { with high-dose } \\
\text { chemotherapy or BMT } \\
\text { studies. }\end{array}$ & none & $\begin{array}{l}\text { faint erythema or dry } \\
\text { desquamation }\end{array}$ & $\begin{array}{l}\text { moderate to brisk } \\
\text { erythema or a patchy } \\
\text { moist desquamation, } \\
\text { mostly confined to skin } \\
\text { folds and creases; } \\
\text { moderate edema }\end{array}$ & $\begin{array}{l}\text { confluent moist } \\
\text { desquamation, } \geq 1.5 \mathrm{~cm} \\
\text { diameter, not confined } \\
\text { to skin folds; pitting } \\
\text { edema }\end{array}$ & $\begin{array}{l}\text { skin necrosis or } \\
\text { ulceration of full } \\
\text { thickness dermis; may } \\
\text { include spontaneous } \\
\text { bleeding not induced by } \\
\text { minor trauma or } \\
\text { abrasion }\end{array}$ \\
\hline $\begin{array}{l}\text { Rash/desquamation } \\
\text { associated with graft versus } \\
\text { host disease (GVHD) for } \\
\text { BMT studies. }\end{array}$ & none & $\begin{array}{l}\text { macular or papular } \\
\text { eruption or erythema } \\
\text { covering <25\% of body } \\
\text { surface area without } \\
\text { associated symptoms }\end{array}$ & $\begin{array}{l}\text { macular or papular } \\
\text { eruption or erythema } \\
\text { with pruritus or other } \\
\text { associated symptoms } \\
\text { covering } \geq 25-<50 \% \text { of } \\
\text { body surface or } \\
\text { localized desquamation } \\
\text { or other lesions } \\
\text { covering } \geq 25-<50 \% \text { of } \\
\text { body surface area }\end{array}$ & $\begin{array}{l}\text { symptomatic } \\
\text { generalized } \\
\text { erythroderma or } \\
\text { symptomatic macular, } \\
\text { papular or vesicular } \\
\text { eruption, with bullous } \\
\text { formation, or } \\
\text { desquamation covering } \\
\geq 50 \% \text { of body surface } \\
\text { area }\end{array}$ & $\begin{array}{l}\text { generalized exfoliative } \\
\text { dermatitis or ulcerative } \\
\text { dermatitis or bullous } \\
\text { formation }\end{array}$ \\
\hline
\end{tabular}




\section{Appendix V (Continued)}

\section{BMT-Specific Adverse Events}

Summary of BMT-Specific Adverse Events that may be used if specified by the protocol. These differ from the standard CTC and may be more relevant to the transplant setting. They are listed here for the convenience of investigators writing transplant protocols. They are also included in the CTC document.

\begin{tabular}{|c|c|c|c|c|c|}
\hline \multicolumn{6}{|c|}{ Grade } \\
\hline Adverse Event & $\mathbf{0}$ & 1 & 2 & 3 & 4 \\
\hline $\begin{array}{l}\text { Stomatitis/pharyngitis } \\
\text { (oral/pharyngeal mucositis) } \\
\text { for BMT studies. }\end{array}$ & none & $\begin{array}{l}\text { painless ulcers, } \\
\text { erythema, or mild } \\
\text { soreness in the absence } \\
\text { of lesions }\end{array}$ & $\begin{array}{l}\text { painful erythema, } \\
\text { edema or ulcers but can } \\
\text { swallow }\end{array}$ & $\begin{array}{l}\text { painful erythema, } \\
\text { edema, or ulcers } \\
\text { preventing swallowing } \\
\text { or requiring hydration } \\
\text { or parenteral (or enteral) } \\
\text { nutritional support }\end{array}$ & $\begin{array}{l}\text { severe ulceration } \\
\text { requiring prophylactic } \\
\text { intubation or resulting } \\
\text { in documented } \\
\text { aspiration pneumonia }\end{array}$ \\
\hline $\begin{array}{l}\text { Transfusion: Platelets for } \\
\text { BMT studies. }\end{array}$ & none & $\begin{array}{l}1 \text { platelet transfusion in } \\
24 \text { hours }\end{array}$ & $\begin{array}{l}2 \text { platelet transfusions in } \\
24 \text { hours }\end{array}$ & $\begin{array}{l}\geq 3 \text { platelet transfusions } \\
\text { in } 24 \text { hours }\end{array}$ & $\begin{array}{l}\text { platelet transfusions and } \\
\text { other measures required } \\
\text { to improve platelet } \\
\text { increment; platelet } \\
\text { transfusion } \\
\text { refractoriness associated } \\
\text { with life-threatening } \\
\text { bleeding. (e.g., HLA or } \\
\text { cross matched platelet } \\
\text { transfusions) }\end{array}$ \\
\hline $\begin{array}{l}\text { Transfusion: pRBCs for } \\
\text { BMT studies. }\end{array}$ & none & $\begin{array}{l}\leq 2 \mathrm{u} \text { pRBC in } 24 \text { hours } \\
\text { elective or planned }\end{array}$ & $\begin{array}{l}3 \mathrm{u} \text { pRBC in } 24 \text { hours } \\
\text { elective or planned }\end{array}$ & $\geq 4 \mathrm{u} \mathrm{pRBC}$ in 24 hours & $\begin{array}{l}\text { hemorrhage or } \\
\text { hemolysis associated } \\
\text { with life-threatening } \\
\text { anemia; medical } \\
\text { intervention required to } \\
\text { improve hemoglobin }\end{array}$ \\
\hline $\begin{array}{l}\text { Transfusion: pRBCs for } \\
\text { pediatric BMT studies. }\end{array}$ & none & $\begin{array}{l}\leq 15 \mathrm{~mL} / \mathrm{kg} \text { in } 24 \text { hours } \\
\text { elective or planned }\end{array}$ & $\begin{array}{l}>15-\leq 30 \mathrm{~mL} / \mathrm{kg} \text { in } 24 \\
\text { hours elective or } \\
\text { planned }\end{array}$ & $>30 \mathrm{~mL} / \mathrm{kg}$ in 24 hours & $\begin{array}{l}\text { hemorrhage or } \\
\text { hemolysis associated } \\
\text { with life-threatening } \\
\text { anemia; medical } \\
\text { intervention required to } \\
\text { improve hemoglobin }\end{array}$ \\
\hline $\begin{array}{l}\text { Thrombotic } \\
\text { microangiopathy (e.g., } \\
\text { thrombotic } \\
\text { thrombocytopenic } \\
\text { purpura/TTP or hemolytic } \\
\text { uremic syndrome/HUS) for } \\
\text { BMT studies. }\end{array}$ & - & $\begin{array}{l}\text { evidence of RBC } \\
\text { destruction } \\
\text { (schistocytosis) without } \\
\text { clinical consequences }\end{array}$ & $\begin{array}{l}\text { evidence of RBC } \\
\text { destruction with } \\
\text { elevated creatinine }(\leq 3 \\
\text { x ULN) }\end{array}$ & $\begin{array}{l}\text { evidence of RBC } \\
\text { destruction with } \\
\text { creatinine }(>3 \times \text { ULN) } \\
\text { not requiring dialysis }\end{array}$ & $\begin{array}{l}\text { evidence of } \mathrm{RBC} \\
\text { destruction with renal } \\
\text { failure requiring } \\
\text { dialysis and/or } \\
\text { encephalopathy }\end{array}$ \\
\hline $\begin{array}{l}\text { Weight gain associated with } \\
\text { Veno-Occlusive Disease } \\
\text { (VOD) for BMT studies. }\end{array}$ & $<2 \%$ & $\geq 2-<5 \%$ & $\geq 5-<10 \%$ & $\geq 10 \%$ or as ascites & $\begin{array}{l}\geq 10 \% \text { or fluid retention } \\
\text { resulting in pulmonary } \\
\text { failure }\end{array}$ \\
\hline
\end{tabular}


CTC Version 2.0

Publish Date: April 30, 1999

\section{Appendix VI}

\section{BMT Complex/Multicomponent Events}

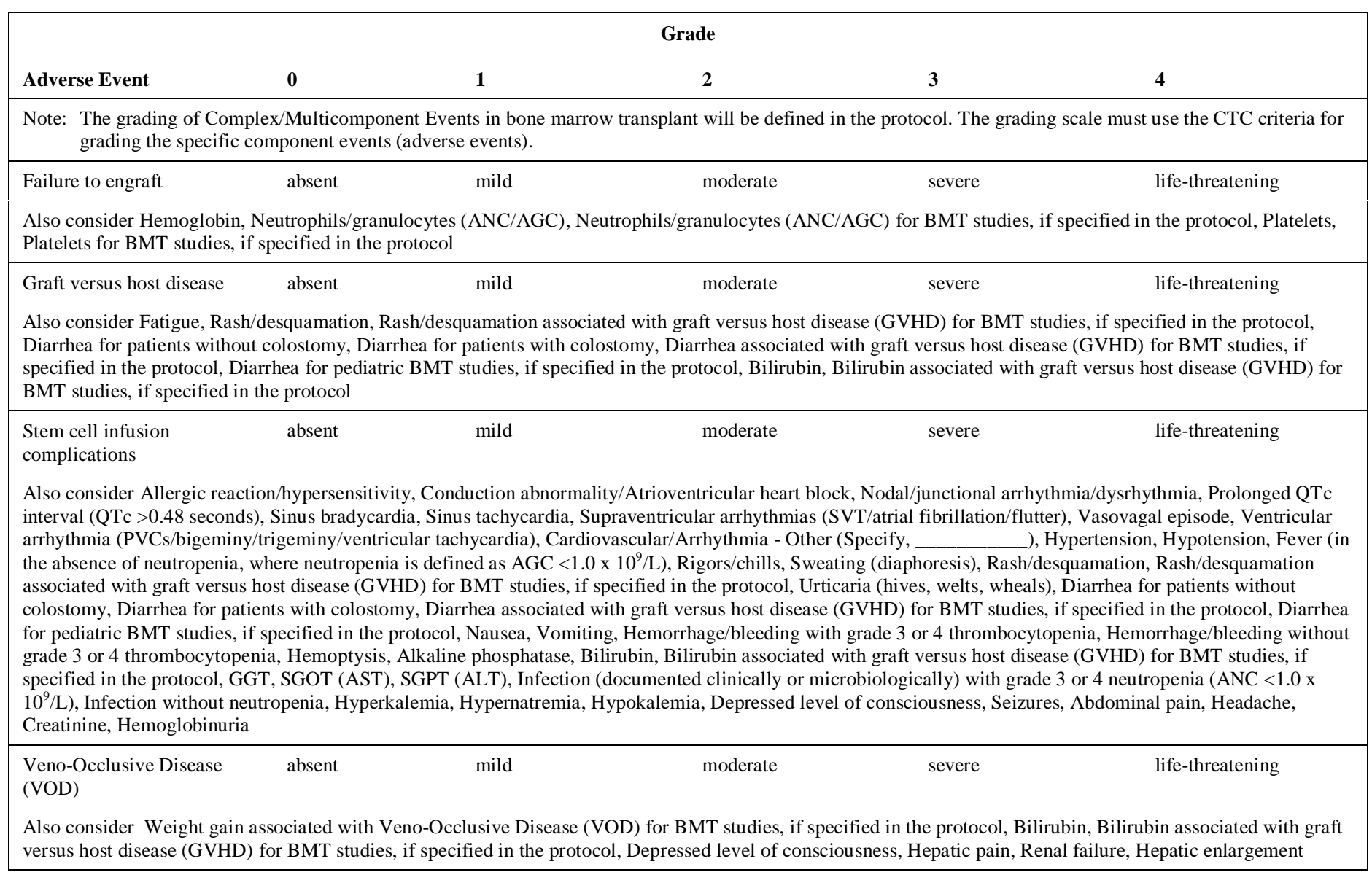

